\title{
Empacotamento e contagem em digrafos: cenários aleatórios e extremais
}

\author{
Roberto Freitas Parente
}

TESE APRESENTADA

$\mathrm{AO}$

Instituto DE MatemáticA E Estatística

DA

Universidade DE SÃo PAUlo

PARA

OBTENÇÃO DO TÍTULO

$\mathrm{DE}$

Doutor EM CIÊNCIAS

Programa: Ciência da Computação

Orientadora: Profa. Dra. Cristiane Maria Sato

Durante o desenvolvimento deste trabalho o autor recebeu auxílio financeiro do CNPq

São Paulo, dezembro de 2016 


\title{
Empacotamento e contagem em digrafos: cenários aleatórios e extremais
}

\author{
Esta versão da tese contém as correções e alterações sugeridas \\ pela Comissão Julgadora durante a defesa da versão original do trabalho, \\ realizada em 27/10/2016. Uma cópia da versão original está disponível no \\ Instituto de Matemática e Estatística da Universidade de São Paulo.
}

Comissão Julgadora:

- Prof $^{a}$. Dr ${ }^{\mathrm{a}}$. Cristiane Maria Sato (orientadora) - UFABC

- Prof. Dr. Yoshiharu Kohayakawa - IME-USP

- Prof. Dr. Fabricio Siqueira Benevides - UFC

- Prof. Dr. Robert David Morris - IMPA

- Prof. Dr. Simon Tichard Griffiths - PUC-RJ 


\section{Agradecimentos}

Agradeço à minha família representada pela minha mãe Águida Freitas Parente e meu pai Francisco Antônio Camelo Parente, por sempre me apoiarem pensando no meu melhor e nunca medirem esforços para que eu tivesse uma educação de qualidade e ser possível chegar à conclusão do meu doutorado.

À minha orientadora Cristiane Maria Sato que após a minha qualificação me "adotou" para a segunda metade do doutorado possibilitando um ritmo de trabalho intenso e profícuo. Sem a sua dedicação e orientação no dia a dia certamente não teria sido possível o término do presente trabalho. Também ao meu orientador da primeira metade do doutorado Yoshiharu Kohayakawa pela paciência e exemplo de pesquisador a ser seguido. Além dos meus colaboradores Carlos Hoppen e Leonardo Coregliano pelos dias de trabalho conjunto que permitiram meu amadurecimento acadêmico.

Ao meu amigo Guilherme Mota, pois além da verdadeiríssima amizade também foi praticamente um coorientador sempre disponibilizando tempo para me ajudar, compartilhar experiências e virar noites tanto no laboratório quanto em bares quando necessário. Sem sua ajuda a conclusão do doutorado seria muito mais difícil. Aproveito e estendo o agradecimento aos diversos membros do laboratório LOCA (Laboratório de Otimização, Combinatória e Algoritmos).

À minha amiga, namorada e companheira Laura Ossei Daltro Dias por toda a sua paciência, compreensão, carinho e por ser meu porto seguro para que fosse possível superar todo o estresse e tensão do doutorado tornando meu dia a dia mais alegre e belo. Além da sua garra na militância da movimento negro que me ensinou muito e contribuiu para ampliar minha compreensão de mundo para além da universidade.

Aos meus amigos e amigas que sempre estiveram dispostos a me ajudar, me apoiar e me xingar quando necessário tanto nos momentos mais difíceis quanto nos alegres, tais como Antô- 
nio Cícero (Tonhão), Antonio Josefran (Zefran), Fábio Sousa (Fábio preto), Flávio Cardoso (Flavinho), Leonardo Carvalho (Leozim), Marcel Segalla (Osama), Natalia Albuquerque (Naty peitão), Lázaro Rocha (Lazim), Roseane Nascimento (Pororoca) e outras pessoas que, apesar de não estarem nominalmente aqui, não foram menos importantes para a conclusão deste trabalho.

À projete liberdade capoeira, representada pelo Mestre Gladson, que por muitas vezes serviu com válvula de escape para as dificuldades do dia a dia, para subjetivar a vida e ajudar a compreender como a cultura popular e negra é fundamental na nossa sociedade. Além que contribuir para a compreensão de que o conhecimento acadêmico não está acima do conhecimento popular, mas que ambos devem caminhar juntos para que assim tenhamos um local mais plural e melhor de se viver.

Por fim, agradeço a todas as pessoas que conheci que contribuiram de alguma maneira para minha formação acadêmica e sociocultural, e a quem mais achar que deva estar aqui :-).

Segue poema do conterrâneo Patativa do Assaré, pois nunca devemos negar ou subjugar nossas origens.

\section{Sou cabra da peste}

Eu sou de uma terra que o povo padece

Mas nunca esmorece, procura vencê,

Da terra adorada, que a bela caboca

De riso na boca zomba no sofrê.

Não nego meu sangue, não nego meu nome,

Olho para fome e pergunto: o que há?

Eu sou brasilêro fio do Nordeste,

Sou cabra da peste, sou do Ceará.

Tem munta beleza minha boa terra,

Derne o vale à serra, da serra ao sertão.

Por ela eu me acabo, dou a própria vida,

É terra querida do meu coração.

Meu berço adorado tem bravo vaquêro

E tem jangadêro que domina o má.

Eu sou brasilêro fio do Nordeste,

Sou cabra da peste, sou do Ceará.
Ceará valente que foi munto franco

Ao guerrêro branco Soare Moreno,

Terra estremecida, terra predileta

Do grande poeta Juvená Galeno.

Sou dos verde mare da cô da esperança,

Que as água balança pra lá e pra cá.

Eu sou brasilêro fio do Nordeste,

Sou cabra da peste, sou do Ceará.

Ninguém me desmente, pois, é com certeza,

Quem qué vê beleza vem ao Cariri,

Minha terra amada pissui mais ainda,

A muié mais linda que tem o Brasí.

Terra da jandaia, berço de Iracema,

Dona do poema de Zé de Alencá.

Eu sou brasilêro fio do Nordeste,

Sou cabra da peste, sou do Ceará.

Patativa do Assaré 


\section{Resumo}

Parente, R. F. Empacotamento e contagem em digrafos: cenários aleatórios e extremais. 2016. Tese (Doutorado) - Instituto de Matemática e Estatística, Universidade de São Paulo, São Paulo, 2016.

Nesta tese estudamos dois problemas em digrafos: um problema de empacotamento e um problema de contagem. Estudamos o problema de empacotamento máximo de arborescências no digrafo aleatório $\mathcal{D}(n, p)$, onde cada possível arco é inserido aleatoriamente ao acaso com probabilidade $p=p(n)$. Denote por $\lambda(\mathcal{D}(n, p))$ o maior inteiro possível $\lambda \geq 0$ tal que, para todo

$0 \leq \ell \leq \lambda$, temos $\sum_{i=0}^{\ell-1}(\ell-i)\left|\left\{v: d^{i n}(v)=i\right\}\right| \leq \ell$. Provamos que a quantidade máxima de arborescências em $\mathcal{D}(n, p)$ é $\lambda(\mathcal{D}(n, p))$ assintoticamente quase certamente. Nós também mostramos estimativas justas para $\lambda(\mathcal{D}(n, p))$ para todo $p \in[0,1]$. As principais ferramentas que utilizamos são relacionadas a propriedades de expansão do $\mathcal{D}(n, p)$, o comportamento do grau de entrada do digrafo aleatório e um resultado clássico de Frank que serve como ligação entre subpartições em digrafos e a quantidade de arborescências. Para o problema de contagem, estudamos a densidade de subtorneios fortemente conexos com 5 vértices em torneios grandes. Determinamos a densidade assintótica máxima para 5 torneios bem como as famílias assintóticas extremais de cada torneios. Como subproduto deste trabalho caracterizamos torneios que são "blow-ups" recursivos de um circuito orientado com 3 vértices como torneios que proíbem torneios específicos de tamanho 5. Como principal ferramenta para esse problema utilizados a teoria de álgebra de flags e configurações combinatórias obtidas através do método semidefinido.

Palavras-chave: digrafos aleatórios, combinatória extremal, arborescência, torneios, álgebra de flags. 


\section{Abstract}

PARENTE, R. F. Packing and counting in digraphs: extremal and random settings. 2016. Tese (Doutorado) - Instituto de Matemática e Estatística, Universidade de São Paulo, São Paulo, 2016.

In this thesis we study two problems dealing with digraphs: a packing problem and a counting problem. We study the problem of packing the maximum number of arborescences in the random digraph $\mathcal{D}(n, p)$, where each possible arc is included uniformly at random with probability $p=p(n)$. Let $\lambda(\mathcal{D}(n, p))$ denote the largest integer $\lambda \geq 0$ such that, for all $0 \leq \ell \leq \lambda$, we

have $\sum_{i=0}^{\ell-1}(\ell-i)\left|\left\{v: d^{i n}(v)=i\right\}\right| \leq \ell$. We show that the maximum number of arc-disjoint arborescences in $\mathcal{D}(n, p)$ is $\lambda(\mathcal{D}(n, p))$ asymptotically almost surely. We also give tight estimates for $\lambda(\mathcal{D}(n, p))$ for every $p \in[0,1]$. The main tools that we used were expansion properties of random digraphs, the behavior of in-degree of random digraphs and a classic result by Frank relating subpartitions and number of arborescences. For the counting problem, we study the density of fixed strongly connected subtournaments on 5 vertices in large tournaments. We determine the maximum density asymptotically for five tournaments as well as unique extremal sequences for each tournament. As a byproduct of this study we also characterize tournaments that are recursive blow-ups of a 3-cycle as tournaments that avoid three specific tournaments of size 5. We use the theory of flag algebras as a main tool for this problem and combinatorial settings obtained from semidefinite method.

Keywords: random digraphs, extremal combinatorics, arborescence, tournament, flag algebras. 


\section{Sumário}

1 Introdução 1

$\begin{array}{lll}2 & \text { Preliminares } & 7\end{array}$

2.1 Notação assintótica . . . . . . . . . . . . . . . . . . . . 7

2.2 Definições básicas . . . . . . . . . . . . . . . . . . . . . 8

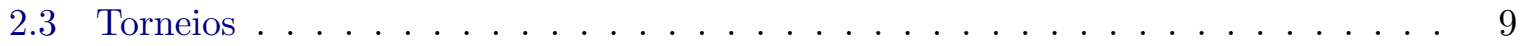

2.4 Digrafo e torneio aleatório . . . . . . . . . . . . . . . . . 11

2.5 Propriedades de variáveis aleatórias . . . . . . . . . . . . . . . . 11

2.6 Princípio da subsubsequência $\ldots \ldots \ldots \ldots \ldots \ldots \ldots$

3 Empacotamento de arborescências em digrafos aleatórios 15

3.1 Introdução . . . . . . . . . . . . . . . . . . . . 15

3.2 Resultado principal . . . . . . . . . . . . . . . . . 16

3.3 Comportamento dos graus em digrafos aleatórios . . . . . . . . . . . . . . . 19

3.4 Estimando $\lambda(\mathcal{D}(n, p)) \ldots \ldots \ldots \ldots \ldots \ldots \ldots \ldots$

3.5 Propriedades de expansão $\ldots \ldots \ldots \ldots \ldots \ldots \ldots$

3.6 Prova do resultado principal . . . . . . . . . . . . . . . . . . . . 32

4 Densidade máxima de subtorneios fortemente conexos $\quad 37$

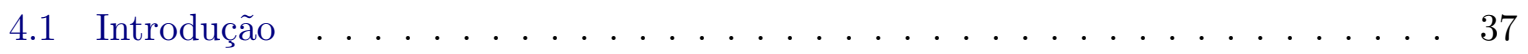

4.2 Resultados . . . . . . . . . . . . . . . . . . . . 39

4.3 Álgebra de flags . . . . . . . . . . . . . . . . . . . . . . . . . 42

4.3 .1 Definições básicas e propriedades . . . . . . . . . . . . . . . 42

$4.3 .2 \quad \mathrm{O}$ método semidefinido . . . . . . . . . . . . . . . . . 44 
4.3.3 Extraindo mais informações do método semidefinido . . . . . . . . . . . . 49

4.3 .4 Torneios, tipos e flags usados . . . . . . . . . . . . . . 52

4.4 Limitantes inferiores . . . . . . . . . . . . . . . . . . . . 54

4.5 Limitantes superiores . . . . . . . . . . . . . . . . . . 57

4.6 Unicidade . . . . . . . . . . . . . . . . . . . . . . . . 59

4.6 .1 Unicidade quase-aleatória . . . . . . . . . . . . . . . . . . . 60

4.6 .2 Unicidade quase-carrossel . . . . . . . . . . . . . . . . . . . . . 61

4.6 .3 Unicidade quase-triangular . . . . . . . . . . . . . . . 65

4.6 .4 Prova do Teorema $4.6 .10 \ldots \ldots \ldots \ldots$

4.6 .5 Prova do Lema $4.6 .19 \ldots \ldots \ldots$. . . . . . . . . . . . . . 78

$\begin{array}{llr}5 & \text { Considerações finais } & 87\end{array}$

5.1 Arborescência . . . . . . . . . . . . . . . . . . . . 87

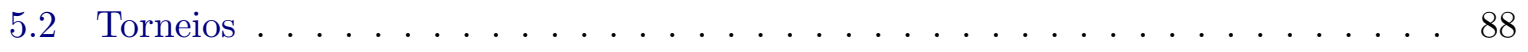

$\begin{array}{ll}\text { A Matrizes positivas semidefinidas } & 91\end{array}$

B Polinômios característicos das matrizes positivas semidefinidas $\quad 97$

$\begin{array}{ll}\text { Referências Bibliográficas } & 99\end{array}$ 


\section{Capítulo 1}

\section{Introdução}

O título deste trabalho nos remete aos dois tipos de problemas com que trabalhamos: problemas de empacotamento e contagem. Em ambos os problemas estamos interessados em contar a quantidade de subestruturas menores dentro de estruturas maiores. A diferença entre eles é que em problemas de empacotamento estamos interessados em contar a quantidade de subestruturas disjuntas e em problemas de contagem estamos interessados em contar a quantidade de cópias das subestruturas independentemente de serem disjuntas.

O nosso primeiro problema, estudado no Capítulo 3, é um problema de empacotamento. Existem basicamente dois tipos de problemas de empacotamento: empacotamento de estruturas geradoras e de não geradoras. Por simplicidade, usaremos o exemplo de grafos, mas tais ideias podem ser estendidas para outras estruturas combinatórias.

Como grafos geradores têm o mesmo conjunto de vértices, em empacotamentos de grafos geradores, procuramos maximizar a quantidade de subestruturas disjuntas nas arestas.

O estudo sobre empacotamentos de árvores geradoras em um grafo (EAG) é um problema clássico de otimização combinatória. Um dos primeiros resultados para esse problema é uma relação de min-max provada por Tutte [Tut61] e Nash-Williams [NW61]. Tal resultado afirma que o tamanho máximo do empacotamento de tais árvores é o menor valor, sob todas as partições $\mathcal{P}$ do conjunto de vértices, da razão entre a quantidade de arestas que cruzam as partes da partição $\mathcal{P}$ e o valor $|\mathcal{P}|-1$. São conhecidos algoritmos eficientes para encontrar o empacotamento de tamanho máximo. Vale mencionar que o resultado acima tem uma prova via matróides, que nos dá um algoritmo eficiente automaticamente.

Uma questão natural é analisarmos tal problema no contexto de digrafos (grafos direcio- 
nados). Desta forma, o análogo em digrafos é quando estamos interessados em estudar o empacotamento de arborescências em digrafos (EAD). Grosseiramente falando, dizemos que uma arborescência é uma árvore geradora direcionada com os arcos "apontando para fora" da raiz.

Assim como o problema de EAG, o problema de EAD também tem um papel importante na ciência da computação quando estamos trabalhando com redes de tráfego e segurança como pode ser visto em [FLST15]. Também existem resultados recentes sobre redes temporais [KK15], onde a direção dos arcos correspondem a uma modelagem desse tipo de rede. Devido a sua importância, o problema de arborescência, e algumas generalizações relacionadas, tem sido bastante estudado em versões algorítmicas como pode ser visto em [GM98, Kam14, LRW15, LLR15]

Por outro lado, quando trabalhamos com empacotamento de grafos não geradores estamos procurando empacotar subgrafos que têm a quantidade de vértices menor do que os grafos onde procuramos empacotar. Como exemplo, o problema clássico do emparelhamento máximo pode ser visto como o empacotamento de arestas disjuntas nos vértices. Assim como os resultados de Menger [Men27] e Mader [Mad78] sobre empacotamento de caminhos. Uma vasta gama de resultados clássicos em otimização combinatória estão dentro desse arcabouço. Para mais problemas e resultados de empacotamento sugerimos o livro de Cornuéjols [Cor01].

Uma direção natural é estudarmos empacotamentos em estruturas aleatórias tais como o grafo aleatório $\mathcal{G}(n, p)$ ou o digrafo aleatório $\mathcal{D}(n, p)$. Quando trabalhamos com grafos aleatórios estamos ignorando os grafos que têm comportamentos incomuns, ou seja, focamos no comportamento da "maioria" dos grafos com uma quantidade fixa de vértices.

Isso é importante pelo fato de que alguns grafos têm uma estrutura bastante específica que aparecem apenas para um conjunto ínfimo de grafos. Informalmente podemos dizer que ao trabalharmos com grafos aleatórios obtemos um panorama geral do conjunto de todos os grafos ao ignorarmos os grafos com estruturas "estranhas".

Uma outra característica importante dos grafos aleatórios é que fixamos um valor $p \in[0,1]$ para a probabilidade de cada uma das arestas estarem presente no mesmo. Desta forma, a possibilidade de variarmos o $p$ no intervalo $[0,1]$, nos dá uma ideia evolutiva do comportamento geral dos grafos com relação a adição de arestas.

Outro tipo de problema de empacotamento de estruturas geradoras em grafos (digrafos) aleatórios que tem sido bastante estudado é o problema de empacotamento de circuitos hamiltonianos (ver [BSKS11, Bol84, BF85, FNP+15, FK08, KKO15, KS12, KO14]). 
No Capítulo 3 estudamos o problema do empacotamento de arborescências disjuntas no digrafo aleatório $\mathcal{D}(n, p)$. Ademais, denotamos por $\tau(\mathcal{D}(n, p))$ a quantidade máxima de arborescências disjuntas nos $\operatorname{arcos}$ em $\mathcal{D}(n, p)$.

Nosso resultado afirma que, com alta probabilidade, $\tau(\mathcal{D}(n, p))=\lambda(\mathcal{D}(n, p))$, onde $\lambda(\mathcal{D}(n, p))$ é o maior inteiro $\lambda \geq 0$ tal que, para todo $0 \leq \ell \leq \lambda, \sum_{i=0}^{\ell-1}(\ell-i)\left|\left\{v: d_{\mathcal{D}(n, p)}^{-}(v)=i\right\}\right| \leq \ell$. Ademais, estimamos de forma justa que o valor de $\lambda(\mathcal{D}(n, p))$ está próximo ao grau de entrada mínimo para todo $p \in[0,1]$.

Uma característica interessante do nosso resultado é que provamos que $\tau(\mathcal{D}(n, p))$ tem uma forte relação com a quantidade de vértices com grau de entrada baixo, o que difere do caso de grafos não orientados apresentado em [GPGS14]. Assim, observamos que os obstáculos de empacotar arborescências em digrafos aleatórios são mais intrincados. Isto acontece devido ao fato de que a raiz de uma arborescência desempenha um papel especial.

Em nosso caso, a razão do porquê $\lambda(\mathcal{D}(n, p))$ é um limitante superior para $\tau(\mathcal{D}(n, p))$ é que, para empacotar $\ell$ arborescências, todo vértice de $\mathcal{D}(n, p)$ cujo grau de entrada é $\ell-i$ deve ser a raiz de pelo menos $i$ arborescências uma vez que seu grau de entrada estaria esgotado.

Similarmente ao caso não direcionado, o núcleo da nossa prova baseia-se em um resultado de otimização combinatória provado por Frank [Fra79] que é similar ao resultado de Tutte e Nash-Williams para o contexto de digrafos. Porém, em vez de tratar com partições, o resultado de Frank impõe condições sobre subpartições.

O nosso segundo problema, estudado no Capítulo 4, é um problema extremal de contagem. Neste caso, estamos interessados em contar a quantidade máxima ${ }^{1}$ de cópias de subestruturas com alguma propriedade desejada em estruturas maiores. Esse tipo de problema está relacionado aos problemas mais clássicos da combinatória extremal tais como os problemas de Turán. Dados inteiros $k$ e $n$, onde $0<k<n$, o problema clássico de Turán é contar a quantidade máxima de arestas em um grafo com $n$ vértices tal que o mesmo não contém cópia do grafo completo de tamanho $k$. Para mais informações sobre problemas de Turán para grafos, hipergrafos e digrafos, recomendamos as resenhas [BES73, BS84, Fur91, Kee11, MV16].

Um desafio a mais do que contar a quantidade máxima de subestruturas é descobrirmos qual é a sua família extremal. Dizemos que uma família é extremal quando o elementos dessa são os

\footnotetext{
${ }^{1}$ Claramente é possível procurarmos minimizar a quantidade de estruturas, mas por simplicidade não abordaremos problemas de minimização no texto.
} 
que maximizam o problema de interesse. Como no exemplo anterior, para o problema clássico de Turán, temos que o grafo $(k-1)$-partido completo é extremal para o problema de contar a quantidade máxima de arestas evitando o grafo completo de tamanho $k$.

No Capítulo 4, estudamos o problema de contar a quantidade máxima de um torneio fortemente conexo fixo de tamanho 5 em torneios de tamanho $n$. Tal problema está relacionado com a família de problemas de Turán em torneios. Um torneio com $n$ vértices é um digrafo obtido através da orientação das arestas de um grafo completo. Dizemos que um torneio é fortemente conexo se existe um caminho direcionado entre todo par de vértices.

Em 1964, Colombo [Col64] provou qual o torneio que maximiza a quantidade de circuitos orientados de tamanho 4. Um ano mais tarde, Beineke e Harary [BH65] estenderam esse resultado provando que o mesmo torneio também maximiza a quantidade de subtorneios fortemente conexos com tamanho fixo.

Durante o nosso trabalho analisamos separadamente os seis torneios fortemente conexos de tamanho 5. Encontramos a densidade máxima assintótica e provamos a unicidade das famílias assintóticas extremais para 5 dos 6 torneios fortemente conexos com tamanho 5 . Caracterizamos a família assintótica extremal através da convergência de $\left(T_{n}\right)_{n \in \mathbb{N}}$ a um certo objeto limite. Para tal, utilizamos a teoria de álgebra de flags, desenvolvida por Razborov [Raz07], que define o objeto limite como o estudo da sequência $(\phi(T))_{T}$, onde $\phi$ é o limite da sequência $\forall T, p\left(T ; T_{n}\right)_{n \in \mathbb{N}}$ e $p\left(T ; T_{n}\right)$ denota a densidade não rotulada de $T$ em $T_{n}$.

$\mathrm{Na}$ teoria de álgebra de flags criamos uma álgebra onde os elementos tem como base a densidade assintótica capturada pela convergência de $p\left(T ; T_{n}\right)$ quando $n \rightarrow \infty$. Chamamos de flag os elementos básicos da nossa álgebra. No contexto deste trabalho, uma flag é um torneio parcialmente rotulado, onde tais rótulos são capturados pelo que chamamos de um tipo $\sigma$. Desta forma, dado um tipo $\sigma$, denotamos por $\mathcal{A}^{\sigma}$ a álgebra das $\sigma$-flags. Para tal, é definido o produto entre dois elementos de $\mathcal{A}^{\sigma}$ de modo que $F_{1} \cdot F_{2}=\sum_{F \in \mathcal{F}_{\ell}^{\sigma}} p\left(F_{1}, F_{2} ; F\right) F$, onde $F_{1}$ e $F_{2}$ são elementos de $\mathcal{A}^{\sigma}$.

Tendo definido a álgebra $\mathcal{A}^{\sigma}$, Razborov provou que os objetos limites $\phi$ são os homomorfismos positivos entre $\mathcal{A}^{\sigma}$ e o conjunto $\mathbb{R}$, denotado por $\operatorname{Hom}^{+}\left(\mathcal{A}^{\sigma}, \mathbb{R}\right)$ o conjunto de tais homomorfismos. O problema da densidade assintótica máxima no contexto da teoria de álgebra de flags é caracterizado por $\max \left\{\phi(T): \phi \in \operatorname{Hom}^{+}\left(\mathcal{A}^{0}, \mathbb{R}\right)\right\}$, onde $\sigma=0$ significa que os torneios são não rotulados. 
A partir da álgebra $\mathcal{A}^{\sigma}$ utilizamos uma noção de pré-ordem para construirmos limitantes superiores para o problema de maximizar a densidade assintótica de $T$. A ideia é utilizamos a relação $T \leq T+g=\sum_{T^{\prime} \in \mathcal{T}_{\ell}}\left(p\left(T ; T^{\prime}\right)+p\left(g ; T^{\prime}\right)\right) T^{\prime}$. Desta forma, escolhemos $g$ de modo que $p\left(g ; T^{\prime}\right)$ tenha valor negativo quando $p\left(T ; T^{\prime}\right)$ for grande e $p\left(g ; T^{\prime}\right)$ suficientemente positivo quando $p\left(T, T^{\prime}\right)$ for pequeno. A escolha de $g$ é feita pela construção de um programa semidefinido e a partir daí utilizamos algumas técnicas posteriormente discutidas para obtermos uma prova matemática para tal.

Após termos provados as densidades assintóticas, conseguimos obter informações sobre a estrutura das famílias assintóticas extremais analisando as restrições do programa semidefinido.

Como subproduto do nosso trabalho de torneios utilizando a teoria de álgebra de flags, caracterizamos o que chamamos de propriedades quase-triangulares que caracterizam os torneios que têm como família assintótica extremal o "blow-up" recursivo de circuitos direcionados com 3 vértices.

O presente trabalho está organizado da seguinte forma: no Capítulo 2, apresentamos as notações e definições básicas que sustentarão o restante do trabalho. Decidimos colocar as definições todas juntas para facilitar ao leitor onde encontrá-las, mas para a comodidade do leitor relembramos as definições em alguns momentos. No Capítulo 3, apresentamos o nosso trabalho referente ao empacotamento de arborescências em digrafos aleatórios. No Capítulo 4, apresentamos o nosso trabalho referente a densidade máxima de subtorneios fortemente conexos. Vale ressaltar que a teoria de flag álgebras, utilizada como ferramenta para estudo da densidade dos torneios, é apresentada como uma subseção do capítulo. Por fim, no Capítulo 5 apresentamos problemas em abertos que estão relacionados aos nossos problemas estudados. 


\section{Capítulo 2}

\section{Preliminares}

Neste capítulo apresentaremos alguns conceitos, definições básicas e exemplo que serão utilizados nos capítulos posteriores. Colocamos esses conceitos agrupados no presente capítulo para que o leitor possa facilmente encontrá-los. Para a comodidade do leitor, em alguns momentos durante os próximos capítulos relembraremos algumas definições quando oportuno.

Vale observar que no Capítulo 4 utilizamos a teoria de álgebra de flags como ferramenta para o nosso resultado, mas, devido a sua extensão, decidimos apresentar a teoria de álgebra de flags localmente no capítulo em que será utilizada.

\subsection{Notação assintótica}

A notação assintótica que utilizaremos pode ser encontrada no Capítulo 1 do Livro [JŁR00]. Decidimos colocar aqui para comodidade do leitor.

Sejam $\left(a_{n}\right)_{n \in \mathbb{N}}$ e $\left(b_{n}\right)_{n \in \mathbb{N}}$ sequências reais tais que $b_{n} \geq 0$ para todo $n$. Usaremos as seguintes notações:

- $a_{n}=O\left(b_{n}\right)$, se existem constantes positivas $C \in \mathbb{R}$ e $N \in \mathbb{N}$ tais que $\left|a_{n}\right| \leq C b_{n}$ para todo $n \geq N$;

- $a_{n}=o\left(b_{n}\right)$, se para todo $\varepsilon>0$ existe $N_{\varepsilon} \in \mathbb{N}$ tal que $\left|a_{n}\right| \leq \varepsilon b_{n}$ para todo $n \geq N_{\varepsilon}$;

- $a_{n}=\Omega\left(b_{n}\right)$, se existem constantes positivas $C \in \mathbb{R}$ e $N \in \mathbb{N}$ tais que $a_{n} \geq C b_{n}$ para todo $n \geq N$;

- $a_{n}=\omega\left(b_{n}\right)$, se para todo $\varepsilon>0$ existe $N_{\varepsilon} \in \mathbb{N}$ tal que $a_{n} \geq(1 / \varepsilon) b_{n}$ para todo $n \geq N_{\varepsilon}$; 
- $a_{n}=\Theta\left(b_{n}\right)$, se existem constantes positivas $C_{1}, C_{2} \in \mathbb{R}$ e $N \in \mathbb{N}$ tais que $C_{1} b_{n} \leq a_{n} \leq C_{2} b_{n}$ para todo $n \geq N$;

- $a_{n} \sim b_{n}$, se para todo $\varepsilon>0$ existe $N_{\varepsilon} \in \mathbb{N}$ tal que $\left|a_{n} / b_{n}-1\right|<\varepsilon$ para todo $n \geq N_{\varepsilon}$.

\subsection{Definições básicas}

Apresentaremos algumas definições básicas de digrafos. Um digrafo $D=(V, A)$ é dado pelo seu conjunto finito de vértices $V$ e seu conjunto $A \subset\{(u, v) \in V \times V: u \neq v\}$ de arcos. Dizemos que um arco $(u, v)$ sai de $u$ e entra em $v$, ou, alternativamente, que $u$ aponta para $v$. Para evitar repetição, fixe $D$ como um digrafo e $v$ como um vértice qualquer de $D$ no restante desta seção.

Seja $X \subset V(D)$, denotamos $\Gamma_{D}^{-}(X)=\{u \in V(D): \exists v \in X,(u, v) \in A(D)\}$ como a vizinhança de entrada de $X$, isto é, o conjunto dos vértices de $D$ que têm arcos "entrando" nos vértices em $X$. Por outro lado, denotamos $\Gamma_{D}^{+}(X)=\{u \in V(D): \exists v \in X,(v, u) \in A(D)\}$ como a vizinhança de saída de $X$, isto é, o conjunto dos vértices de $D$ que têm arcos "saindo" dos vértices em $X$.

Seja $v \in V(D)$ um vértice de $D$, denotamos por $d_{D}^{-}(v)$ o grau de entrada de $v$ que é a quantidade de $\operatorname{arcos}$ de $D$ que "entram" em $v$, ou seja, $d_{D}^{-}(v)=\left|\Gamma_{D}^{-}(v)\right|$. Denotamos por $d_{D}^{+}(v)$ o grau de saída de $v$ que é a quantidade de $\operatorname{arcos}$ de $D$ que "saem" de $v$, ou seja, $d_{D}^{+}(v)=\left|\Gamma_{D}^{+}(v)\right|$. Ademais, definimos $\delta^{-}(D)=\min \left\{d_{D}^{-}(v): v \in V\right\}$ como o grau de entrada mínimo e $\delta^{+}(D)=$ $\min \left\{d_{D}^{+}(v): v \in V\right\}$ como o grau de saída mínimo.

Agora vamos apresentar o conceito de corte em um digrafo. Dado um digrafo $D=(V, A)$ e conjuntos disjuntos, $S, S^{\prime} \subseteq V$, definimos $A_{D}\left(S, S^{\prime}\right)$ como o conjunto de $\operatorname{arcos}(u, v) \in A$ tal que $u \in S$ e $v \in S^{\prime}$. Denotamos por corte $\left[S, S^{\prime}\right]$ a união $\operatorname{dos} \operatorname{arcos} A_{D}\left(S, S^{\prime}\right) \cup A_{D}\left(S^{\prime}, S\right)$.

Seja $S \subseteq V(D)$, denotamos por $D[S]$ o digrafo induzido $S$, o digrafo com conjunto de vértices $S$ e conjunto de $\operatorname{arcos} A_{D}[S]=\{(u, v) \in A(D): u \in S, v \in S\}$. O grafo subjacente do digrafo $D=(V, A)$ é o multigrafo obtido quando ignoramos as orientações dos arcos.

Uma arborescência de $D$ é um digrafo $T=\left(V, A_{T}\right)$ onde $A_{T} \subseteq A$ tal que o grafo subjacente de $T$ é uma árvore geradora e, todo vértices tem grau de entrada 1, exceto por um vértice e este tem grau de entrada zero e será chamado de raiz. Grosseiramente falando, uma arborescência é uma árvore geradora com os arcos "apontando para fora" da raiz. Denotamos por $\tau(D)$ a quantidade máxima de arborescências disjuntas nos arcos em $D$. Alternativamente, dizemos 
que $\tau(D)$ é o número de empacotamento de arborescências disjuntas nos arcos em $D$.

\subsection{Torneios}

O problema que trabalhamos no Capítulo 4 está relacionado ao conceito de torneios. Um torneio é um digrafo obtido a partir da orientação das arestas de um grafo completo. Desta forma, as definições que utilizaremos aqui também valem, em sua maioria, para digrafos, mas por simplicidade definiremos para torneio diretamente. Utilizamos a letra $T$ para denotar um torneio.

Dado um digrafo $T=(V, A)$ com $n$ vértices, dizemos que $T$ é um torneio se, e somente se, para todo par de vértice $u$ e $v$ de $V(T)$ temos que exatamente um dentre $(u, v)$ e $(v, u)$ partence a $A$. Ademais, observe que $|A(T)|=n(n-1) / 2$.

Dizemos que um torneio $T$ é transitivo se, e somente se, para todo par de arco $(x, y) \in A(T)$ e $(y, z) \in A(T)$, temos que $(x, z) \in A(T)$. Um torneio $T$ é localmente transitivo se, para todo vértices $v$ de $T$, a vizinhança de saída de $v$ e a vizinhança de entrada de $v$ são ambas transitivas, isto é, para todo $v \in V(T), T\left[\Gamma^{-}(v)\right]$ e $T\left[\Gamma^{+}(v)\right]$ são torneios transitivos. Alternativamente, um torneio localmente transitivo é um torneio que não contém ocorrências de $W_{4}$ e $L_{4}$, onde $W_{4}$ e $L_{4}$ são os torneios de tamanho 4 com sequências de grau de saída $(1,1,1,3)$ e $(0,2,2,2)$ respectivamente. Dizemos que um torneio é fortemente conexo se, e somente se, para todo par $u$ e $v$ de vértices, existem caminhos direcionados de $u$ até $v$ e de $v$ até $u$. Um torneio $T \operatorname{com} 2 n+1$ vértices ${ }^{1}$ é dito balanceado se todo vértice tem grau de saída $n$.

Com essas definições em mãos, vamos apresentar dois torneios balanceados que serão bastante úteis para nosso estudo no Capítulo 4.

Primeiro observe que, a menos de isomorfismo, só existe um torneio que é localmente transitivo e balanceado com $2 n+1$ vértices. O chamaremos de torneio carrossel e denotamos por $R_{2 n+1}$. Esse torneio tem conjunto de vértices $V\left(R_{2 n+1}\right)=\{0,1, \ldots, 2 n\}$ e conjunto de arcos $A\left(R_{2 n+1}\right)=\left\{(v,(v+i) \bmod (2 n+1)): v \in V\left(R_{2 n+1}\right)\right.$ e $\left.i \in[n]\right\}$, onde $[n]=\{1,2, \ldots, n\}$. Para exemplos, veja a Figura 2.1.

Para definir nosso segundo torneio balanceado $\left(\vec{C}_{n}^{3}\right)$ precisamos primeiramente definir o que são "blow-ups" recursivos do circuito direcionado de 3 vértices $\left(\vec{C}_{3}\right)$. Para todo $n \geq 3$,

\footnotetext{
${ }^{1}$ Por simplicidade, aqui não vamos nos importar com o caso em que $T$ tem $2 n$ vértices.
} 


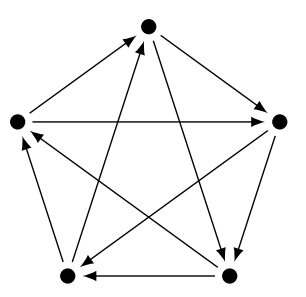

$R_{5}$

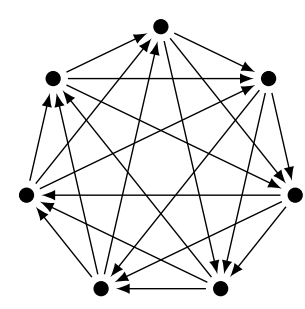

$R_{7}$

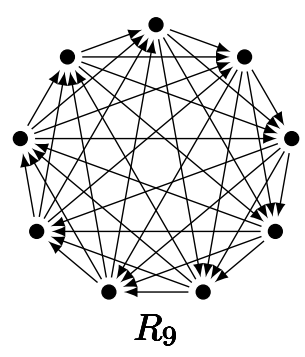

$R_{9}$

Figura 2.1: Torneios carrossel $R_{2 n+1}$ para $n=2,3,4$.

sejam $n_{0} \geq n_{1} \geq n_{2}$ tais que $n_{0}+n_{1}+n_{2}=n$ e $n_{i} \in\{\lfloor n / 3\rfloor,\lceil n / 3\rceil\}$ para todo $i \in\{0,1,2\}$. Defina $A_{0}=\left\{1, \ldots, n_{0}\right\}, A_{1}=\left\{n_{0}+1, \ldots, n_{1}\right\}$ e $A_{2}=\left\{n_{1}+1, \ldots, n_{2}\right\}$. Com isso, denotamos $\vec{C}_{n}^{3}$ como o torneio sobre $[n]$ tal que $(v, w) \in A\left(\vec{C}_{n}^{3}\right)$ para todo $v \in A_{i}$ e $w \in A_{(i+1) \bmod 3}$, e $\vec{C}_{n}^{3}\left[A_{i}\right]$ é isomorfo a $\vec{C}_{n_{i}}^{3}$ para todo $i=0,1,2$ (veja Figura 2.2).

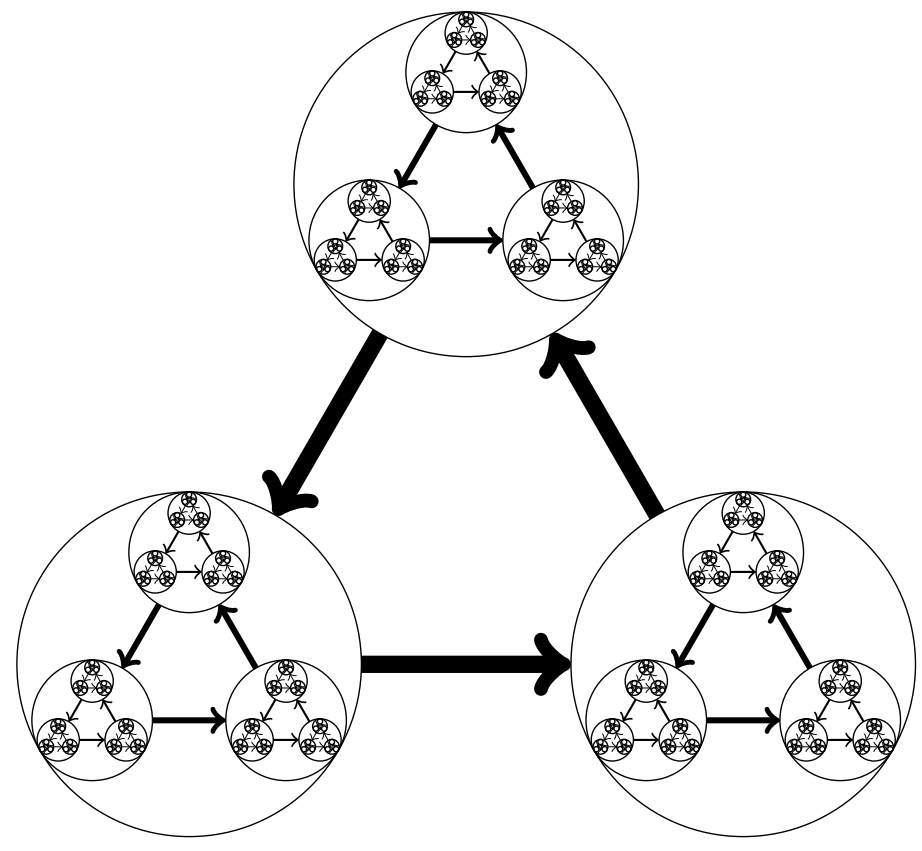

Figura 2.2: Estrutura típica do $\vec{C}_{n}^{3}$.

Para simplificar a notação, dado um digrafo $D$, usaremos $d^{-}(v)$ para denotar $d_{D}^{-}(v)$. Similarmente, $d^{+}(v)=d_{D}^{+}(v), \delta^{-}=\delta^{-}(D), \delta^{+}=\delta^{+}(D), \tau=\tau(D)$ e $\lambda=\lambda(D)$, e assim por diante a menos que seja necessário deixar explícito o digrafo que estamos utilizando. 


\subsection{Digrafo e torneio aleatório}

Para começar, vamos apresentar a noção de digrafo aleatório e torneio aleatório que utilizamos no trabalho.

Dada uma função $p=p(n): \mathbb{R} \rightarrow[0,1]$ e um inteiro positivo $n$, denotamos $\mathcal{D}(n, p)$ como o digrafo aleatório com conjunto de vértices $[n]=\{1,2, \ldots, n\}$ tal que cada um dos $n(n-1)$ arcos são inseridos uniformemente ao acaso com probabilidade $p$.

Já quando falamos de torneios aleatórios, estamos preocupado na orientação dos arcos entre os vértices. Seja $\boldsymbol{R}_{\boldsymbol{n}, \mathbf{1} / \mathbf{2}}$ o torneio aleatório com conjunto de vértices $[n]$ onde cada orientação de um arco é apresentada com probabilidade $1 / 2$ independentemente para todos os pares de vértices.

É um exercício de concentração em variáveis aleatórias binomiais provar que

$$
\forall T \text { torneio, } \lim _{n \rightarrow \infty} p\left(T ; \boldsymbol{R}_{\boldsymbol{n}, \mathbf{1 / 2}}\right)=\mathbb{E}\left[p\left(T ; \boldsymbol{R}_{|\boldsymbol{T}|, \mathbf{1} / \mathbf{2}}\right)\right]=\frac{|T| !}{|\operatorname{Aut}(T)| 2^{\left(\begin{array}{c}
(T) \\
2
\end{array}\right)}},
$$

com alta probabilidade, isto é, a sequência $\left(p\left(T ; \boldsymbol{R}_{\boldsymbol{n}, \mathbf{1} / \mathbf{2}}\right)_{n \in \mathbb{N}}\right.$ é convergente com probabilidade 1 quando $n \rightarrow \infty$.

Por fim, dada uma sequência de espaço de probabilidade $\left(\Omega_{i}, \mathcal{F}_{i}, \operatorname{Pr}_{i}\right)_{i \in \mathbb{N}}$, afirmamos que uma sequência de eventos $\left(A_{i}\right)_{i \in \mathbb{N}}$ vale assintoticamente quase certamente (abreviado por a.q.c.) se $\operatorname{Pr}_{n}\left(A_{n}\right) \rightarrow 1$ quando $n \rightarrow \infty$.

Todos os resultados neste trabalho, exceto se indicado o contrário, o espaço de probabilidade é o definido pelo $\mathcal{D}(n, p)$ e a notação assintótica refere-se a $n$ indo para o infinito.

\subsection{Propriedades de variáveis aleatórias}

Começamos apresentando duas desigualdades para variáveis aleatórias bem conhecidas. Como referência deixamos o primeiro capítulo em [JŁR00]. A primeira é a desigualdade de Markov. Seja $X$ uma variável aleatória e $t>0$ um inteiro. Se $X \geq 0$ quase certamente, então

$$
\operatorname{Pr}(X \geq t) \leq \frac{\mathbb{E}[X]}{t}
$$

Observação 2.5.1. Observe que a Equação (2.1) é bastante útil quando temos que o valor 
esperado de $X$ vai para zero muito rapidamente, i.e., $\mathbb{E}[X]=o(1)$. Assim, para todo inteiro $t>0$, temos $\operatorname{Pr}(X \geq t) \leq \mathbb{E}[X] / t=o(1)$.

A segunda é a desigualdade de Chebyshev. Seja $X$ uma variável aleatória tal que $\mathbb{E}[X]<\infty$ e um real $k>0$, então temos

$$
\operatorname{Pr}(|X-\mathbb{E}[X]| \geq k) \leq \frac{\operatorname{Var}(X)}{k^{2}}
$$

Observação 2.5.2. Observe que quando $X \geq 0$ e $\operatorname{Var}(X)=o\left(\mathbb{E}[X]^{2}\right)$, fazendo $k=\mathbb{E}[X]$ temos pela Equação $(2.2)$ que $\operatorname{Pr}(X=0) \leq \operatorname{Var}(X) / \mathbb{E}[X]^{2}=o(1)$.

\section{Variáveis aleatórias binomiais}

Quando trabalhamos com estruturas combinatórias aleatórias como digrafos e torneios aleatórios temos que muitas propriedades dessas estruturas são capturadas por variáveis aleatórias binomiais. Desta forma, é importante termos boas estimativas para esse tipo de variável aleatória. A seguir temos dois resultados referentes a variáveis aleatórias binomiais.

Teorema 2.5.3 (Limitantes de Chernoff [JŁR00]). Denotamos $X_{1}, \ldots, X_{n}$ como $n$ variáveis de Bernoulli independentes. Sejam $X=\sum_{i=1}^{n} X_{i}$ e $\mu=\mathbb{E}[X]$. Então, para qualquer $0<\tau<1$,

$$
\begin{aligned}
& \operatorname{Pr}(X \geq(1+\tau) \mu) \leq e^{-\tau^{2} \mu / 3}, \\
& \operatorname{Pr}(X \leq(1-\tau) \mu) \leq e^{-\tau^{2} \mu / 2} .
\end{aligned}
$$

Lema 2.5.4 (Lemma 16 [GPGS14]). Para toda constante $\eta>0$, existem constantes positivas $C_{1}$ e $C_{2}$ tais que o seguinte vale para qualquer função $0 \leq p=p(n) \leq 1 / \sqrt{n}$ e todo inteiro $0<k \leq(1-\eta) n p$. Seja $X \sim \operatorname{Bin}(n, p)$. Então,

$$
\operatorname{Pr}(X \leq k)=C\left(\frac{e^{-p n}}{\sqrt{k}}\right)\left(\frac{e p n}{k}\right)^{k}, \operatorname{com} C_{1} \leq C \leq C_{2}
$$

\subsection{Princípio da subsubsequência}

Em várias provas, usamos o princípio da subsubsequência onde afirma que se $x$ é uma constante e $\left(x_{n}\right)_{n \in \mathbb{N}}$ é uma sequência real cuja toda subsequência tem uma subsubsequência 
convergente para $x$, então $x_{n} \rightarrow x$.

Teorema 2.6.1 (Princípio da subsubsequência). Sejam $\left(x_{n}\right)_{n \in \mathbb{N}}$ uma sequência de números reais e $x \in \mathbb{R}$ um ponto fixo. Se, para toda subsequência de $\left(x_{n}\right)_{n \in \mathbb{N}}$, existe uma subsubsequênca que converge a $x$, então a sequência $\left(x_{n}\right)_{n \in \mathbb{N}}$ converge a $x$.

O princípio da subsubsequência é utilizado quando já provamos que $\mathcal{D}(n, p)$ vale para $p=$ $p(n)$ pertence a diversos subintervalos de $[0,1]$ que se sobrepõe e queremos afirmar que vale para qualquer valor de $p$ em $[0,1]$. Aqui vamos dar um exemplo da aplicação deste princípio.

Suponha que estamos trabalhando com o digrafo aleatório $\mathcal{D}(n, p)$ com $p=p(n) \in[0,1]$ e suponha que uma propriedade $A=A_{n}$ vale a.q.c. para $p=O(\log (n) / n)$ e para $p=\omega(\log (n) / n)$. Utilizaremos o princípio da subsubsequência para mostrar que a propriedade $A=A_{n}$ vale a.q.c. para todos os valores possíveis de $p \in[0,1]$ sem restrições.

Considere a sequência $\left(x_{n}\right)_{n \in \mathbb{N}}$ tal que $x_{n}=\operatorname{Pr}\left(A_{n}\right)$ e seja $\left(x_{n_{k}}\right)_{n_{k} \in \mathbb{N}}$ uma subsequência de $\left(x_{n}\right)_{n \in \mathbb{N}}$. Temos os dois casos possíveis

$$
\begin{aligned}
& \limsup _{k \rightarrow \infty} \frac{p\left(n_{k}\right) n_{k}}{\log \left(n_{k}\right)}=\infty \\
& \limsup _{k \rightarrow \infty} \frac{p\left(n_{k}\right) n_{k}}{\log \left(n_{k}\right)}=\alpha, \quad \text { onde } \alpha \text { é uma constante. }
\end{aligned}
$$

Se estamos no primeiro caso (Equação (2.6)), temos que existe uma subsequência $\left(x_{n_{j}}\right)_{n_{j} \in \mathbb{N}}$ de $\left(x_{n_{k}}\right)_{n_{k} \in \mathbb{N}}$ tal que $p\left(n_{j}\right) n_{j} / \log \left(n_{j}\right)=\infty$, então temos que $\operatorname{Pr}\left(A_{n_{j}}\right) \rightarrow 1$, quando $j \rightarrow \infty$, devido a $p\left(n_{k}\right)=\omega\left(\log \left(n_{k}\right) / n_{k}\right)$. Se estamos no segundo caso (Equação (2.7)), temos que existe uma subsequência $\left(x_{n_{j}}\right)_{n_{j} \in \mathbb{N}}$ de $\left(x_{n_{k}}\right)_{n_{k} \in \mathbb{N}}$ tal que $p\left(n_{j}\right) n_{j} / \log \left(n_{j}\right) \leq \alpha$, então temos que $\operatorname{Pr}\left(A_{n_{j}}\right) \rightarrow 1$ quando $k \rightarrow \infty$ nessa subsequência de $\left(x_{n_{k}}\right)_{n_{k} \in \mathbb{N}}$ devido a $p\left(n_{k}\right)=O\left(\log \left(n_{k}\right) / n_{k}\right)$.

Desta forma, pelo princípio da subsubsequência temos que $\left(x_{n}\right)_{n \in \mathbb{N}}$ também converge para 1 , isto é, $A=A_{n}$ vale a.q.c. 


\section{Capítulo 3}

\section{Empacotamento de arborescências em digrafos aleatórios}

Neste capítulo apresentamos nosso resultado sobre o problema de empacotamento de arborescências em digrafos aleatórios. Tal resultado foi obtido em conjunto com Carlos Hoppen e Cristiane M. Sato

\subsection{Introdução}

Muitos problemas importantes em matemática discreta tratam do empacotamento de estruturas com alguma propriedade desejada em estruturas maiores. O objetivo destes problemas são tipicamente encontrar a quantidade máxima possível de tais estruturas disjuntas. Uma vasta gama de resultados clássicos em otimização combinatória estão dentro desse arcabouço. Como exemplo, o problema do emparelhamento máximo pode ser visto como o empacotamento de arestas disjuntas nos vértices. Também destacamos os resultados de Tutte [Tut61] e NashWilliams [NW61] sobre o o problema de empacotamento de árvores geradoras. Assim como os resultados de Menger [Men27] e Mader [Mad78] sobre empacotamento de caminhos. Para mais problemas e resultados de empacotamento sugerimos o livro de Cornuéjols [Cor01].

É natural que exista uma extensa literatura sobre esse tópico dado que existe uma grande quantidade de problemas de empacotamento em combinatória. Por exemplo, o problema de empacotamento de circuitos hamiltonianos em estruturas aleatórias tem sido bastante estudado desde 1980 como pode ser visto em [BF85, Bol84, BSKS11, FK08, KKO15, KO14, KS12]. No 
caso particular de digrafos, alguns resultado significantes têm sido obtidos recentemente [FL16, $\left.\mathrm{FNP}^{+} 15\right]$.

No que diz respeito a arborescências, recentemente, num estudo sobre arborescências arcoíris, Bal, Bennett, Cooper, Frieze e Prałat $\left[\mathrm{BBC}^{+} 16\right]$ provaram que no processo aleatório de digrafos (onde os arcos são adicionados um por um) o momento em que existe um único vértice com grau de entrada zero é o mesmo que o digrafo contém uma arborescência assintoticamente quase certamente.

Organização do capítulo. O presente capítulo está organizado como segue. Na Seção 3.2, apresentamos nosso resultado principal e suas diferenças com o resultado no contexto de grafos aleatórios não orientados. Separamos a prova do nosso resultado principal em três seções. Na Seção 3.3, estudamos propriedades dos graus em $\mathcal{D}(n, p)$. Na Seção 3.4 apresentamos a relação entre $\lambda(\mathcal{D}(n, p))$ e o grau de entrada mínimo. Na Seção 3.5, provamos algumas propriedades de cortes e expansão de $\mathcal{D}(n, p)$. Por último, na Seção 3.6, provamos nosso resultado principal (Teorema 3.2.3).

\subsection{Resultado principal}

Recentemente, Gao, Pérez-Giménez e Sato [GPGS14] obtiveram resultados a respeito do empacotamento de árvores geradoras em grafos aleatórios. Um desses resultados é o Teorema 3.2.1 a seguir.

Teorema 3.2.1 (Pu-Pérez-Giménez-Sato [GPGS14]). Para $p=p(n) \in[0,1]$, a quantidade máxima de árvores geradoras disjuntas nas arestas em $\mathcal{G}(n, p)$ é a.q.c.

$$
\min \left\{\delta(\mathcal{G}(n, p)),\left\lfloor\frac{m(\mathcal{G}(n, p))}{n-1}\right\rfloor\right\}
$$

É fácil ver que $\delta(\mathcal{G}(n, p))$ e $m(\mathcal{G}(n, p)) /(n-1)$ são limitantes superiores para a quantidade de árvores geradoras disjuntas nas arestas, pois toda árvore geradora tem pelo menos uma aresta incidente em todo vértice do grafo e tem exatamente $n-1$ arestas.

O seguinte resultado clássico provado por Tutte e Nash-Williams é a principal ferramenta utilizada em [GPGS14] para provar que o máximo é alcançado por um desses dois parâmetros.

Teorema 3.2.2 (Tutte [Tut61] e Nash-Williams [NW61]). Dado um grafo $G=(V, E)$ e um 
inteiro $k \geq 0, G$ contêm $k$ árvores geradoras disjuntas nas arestas se, e somente se, para toda partição $\mathcal{P}$ de $V$, a quantidade de arestas com extremos em partes distintas é pelo menos $k(|\mathcal{P}|-1)$.

É bastante natural que esse resultado (que é, na verdade, uma relação de min-max) passa ser usado com sucesso para grafos aleatórios, pois a condição sobre partição é essencialmente uma condição de expansão e é bem conhecido que grafos aleatórios têm boas propriedades de expansão.

Nosso resultado do presente capítulo é o análogo ao Teorema 3.2.1 para o contexto de digrafos. De fato, lidamos com o empacotamento de arborescências, que são o análogo a árvores geradoras em digrafos. Como visto no capítulo de preliminares (Capítulo 2), uma arborescência, grosseiramente falando, é uma árvore geradora com os arcos "apontando para fora" da raiz.

Estamos interessado em empacotar o máximo de arborescência no digrafo aleatório $\mathcal{D}(n, p)$, onde $\mathcal{D}(n, p)$ é o digrafo aleatório com conjunto de vértices $[n]$ onde os arcos são inseridos uniformemente ao acaso com probabilidade $p$ independentemente. As definições básicas sobre digrafos podem ser encontradas na Seção 2.2.

Desta forma, denotamos $\tau(\mathcal{D}(n, p)))$ como a quantidade máxima de arborescência disjuntas nos arcos em $\mathcal{D}(n, p)$ e nosso resultado principal é apresentado como segue.

Teorema 3.2.3 (Hoppen-Parente-Sato[HPS16]). Para todo $p=p(n) \in[0,1]$, temos que a.q.c.

$$
\tau(\mathcal{D}(n, p))=\lambda(\mathcal{D}(n, p))
$$

onde $\lambda(\mathcal{D}(n, p))$ é o maior inteiro $\lambda \geq 0$ tal que, para todo $0 \leq \ell \leq \lambda$,

$$
\sum_{i=0}^{\ell-1}(\ell-i)\left|\left\{v: d_{\mathcal{D}(n, p)}^{-}(v)=i\right\}\right| \leq \ell .
$$

Ademais,

(a) se $p=\frac{\log (n)-h(n)}{(n-1)} \operatorname{com} h(n)=\omega(1)$, então $\lambda(\mathcal{D}(n, p))=0$ a.q.c.;

(b) se $p=\frac{\log (n)+h(n)}{(n-1)} \operatorname{com} h(n)=O(\log \log n)$, então $\lambda(\mathcal{D}(n, p)) \in\left\{\delta^{-}(\mathcal{D}(n, p)), \delta^{-}(\mathcal{D}(n, p))+1\right\}$ a.q.c.;

(c) se $p=\frac{\log (n)+h(n)}{(n-1)} \operatorname{com} h(n)=o(\log n)$ e $h(n)=\Omega(\log \log n)$, então $\lambda(\mathcal{D}(n, p)) \sim \delta^{-}(\mathcal{D}(n, p))$ a.q.c. 
Uma característica interessante do nosso resultado é que $\tau(\mathcal{D}(n, p))$ tem uma forte relação com a quantidade de vértices com grau baixo.

Isso defere do caso de grafos não orientados no seguinte sentido. O Teorema 3.2.1 nos diz que, para grafos aleatórios, o obstáculo de empacotar árvores geradora é bastante simples: ou não temos arestas suficientes para obtermos uma quantidade maior de árvores geradoras ou esgotamos as arestas incidentes em um vértice.

Nosso resultado mostra que para digrafos aleatórios os obstáculos de empacotar arborescências são mais intrincado mesmo que ainda seja decorrente de restrições naturais. Isto é devido ao fato de que a raiz de uma arborescência desempenha um papel especial, o que não acontece no contexto de grafos não orientados.

Em nosso caso, a razão do porque $\lambda(\mathcal{D}(n, p))$ é um limitante superior para $\tau(\mathcal{D}(n, p))$ é que, para empacotar $\ell$ arborescências, todo vértice de $\mathcal{D}(n, p)$, cujo grau de entrada é $\ell-i$, deve ser a raiz de pelo menos $i$ arborescências uma vez que seu grau de entrada estaria esgotado.

Similarmente ao caso não direcionado, o núcleo da nossa prova baseia-se em um resultado de otimização combinatória provado por Frank [Fra79] que é similar ao resultado de Tutte e Nash-Williams para o contexto de digrafos. Porém, em vez de tratar com partições, o resultado de Frank impõe condições sobre subpartições. Uma subpartição do conjunto $S$ é um conjunto de subconjuntos de $S$ não vazios dois a dois disjuntos. Observe que, diferentemente da definição de partição, uma subpartição não precisa necessariamente incluir todo elemento de $S$.

Dado um digrafo $D=(V, A)$ e $S \subseteq V$, denotamos $d_{D}^{-}(S)$ como a quantidade de arcos entrando em $S$ (de $V \backslash S$ ) em $D$. Para referências futuras, também denote $d_{D}^{+}(S)$ como a quantidade de arcos saindo de $S$ (para $V \backslash S$ ) em $D$.

Teorema 3.2.4 (Frank [Fra79]). Sejam $D=(V, A)$ um digrafo e $k \geq 0$ um inteiro. Então $D$ contêm $k$ arborescências disjuntas nas arestas se, e somente se, para toda subpartição $\mathcal{P}$ de $V$,

$$
\sum_{U \in \mathcal{P}} d_{D}^{-}(U) \geq k(|\mathcal{P}|-1)
$$

Uma das dificuldades de trabalhar com subpartições em vez de partições é que alguns vértices podem não ser incluídos em alguma parte e seus graus de entradas não contribuem com o somatório na Equação (3.3) que é algo que não acontece no caso de grafos. 


\subsection{Comportamento dos graus em digrafos aleatórios}

Nesta seção, apresentamos alguns resultado sobre o grau de entrada (de saída) mínimo em $\mathcal{D}(n, p)$ e também provamos algumas propriedades dos vértices com grau baixo. O seguinte lema é uma aplicação do Lema 2.5.4 à variável aleatória que determina o grau de entrada (de saída) de $\mathcal{D}(n, p)$. Utilizamos $d^{-/+}(v)$ para denotar ou $d^{-}(v)$ ou $d^{+}(v)$.

Lema 3.3.1. Sejam $\varphi \geq 0.9$ e $0<\eta<1$ contantes. Existem contantes $C_{1}>0$ e $C_{2}>0$ tais que, para qualquer função $\alpha=\alpha(n) \in(0,1-\eta]$ e qualquer função $p$ satisfazendo $0.9 \log n /(n-1) \leq$ $p \leq \varphi \log n /(n-1)$, o seguinte vale:

(i) Existe $C=C(n) \in\left[C_{1}, C_{2}\right]$ tal que para todo $v \in[n]$,

$$
\operatorname{Pr}\left(d^{-/+}(v) \leq \alpha p(n-1)\right)=\frac{C}{\sqrt{\alpha p(n-1)}} \exp \left(-p(n-1)\left(1-\alpha \log \left(\frac{e}{\alpha}\right)\right)\right)
$$

(ii) $\operatorname{Pr}\left(\delta^{-/+} \leq \alpha p(n-1)\right) \leq \frac{C_{2}}{\sqrt{\alpha p(n-1)}} \exp \left(\log n-p(n-1)\left(1-\alpha \log \left(\frac{e}{\alpha}\right)\right)\right)$;

(iii) $\operatorname{Pr}\left(\delta^{-/+}>\alpha p(n-1)\right) \leq \frac{\sqrt{\alpha p(n-1)}}{C_{1}} \exp \left(-\log n+p(n-1)\left(1-\alpha \log \left(\frac{e}{\alpha}\right)\right)\right)$;

(iv) $\operatorname{Pr}\left(\exists v \in[n]: \min \left\{d^{-}(v), d^{+}(v)\right\} \leq \alpha p(n-1)\right)$

$$
\leq \frac{\left(C_{2}\right)^{2}}{\alpha p(n-1)} \exp \left(\log n-2 p(n-1)\left(1-\alpha \log \left(\frac{e}{\alpha}\right)\right)\right) .
$$

Demonstração. A prova de (i)-(iii) é basicamente a mesma que a prova de [GPGS14, Lemma 18]. Incluímos aqui por motivo de completude.

Para todo $v \in V$, seja $d^{-}(V)$ a variável aleatória que determina o grau de entrada de $v$. Observe que $d^{-}(v)$ tem distribuição $\operatorname{Bin}(n-1, p)$. Assim, pelo Lema 2.5.4, existem as contantes $C_{1}$ e $C_{2}$ (dependendo somente de $\eta$ ) e uma constante $C=C(n) \in\left[C_{1}, C_{2}\right]$ tais que

$$
\begin{aligned}
\operatorname{Pr}\left(d^{-}(v) \leq \alpha p(n-1)\right) & =C\left(\frac{e^{-p(n-1)}}{\sqrt{\alpha p(n-1)}}\right)\left(\frac{e}{\alpha}\right)^{\alpha p(n-1)} \\
& =\frac{C}{\sqrt{\alpha p(n-1)}} \exp \left(-p(n-1)\left(1-\alpha \log \left(\frac{e}{\alpha}\right)\right)\right)
\end{aligned}
$$

Isso prova (i). Denotamos por $Y$ a variável aleatória que determina a quantidade de vértices 
$v \in[n]$ tais que $d^{-}(v) \leq \alpha p(n-1)$. Então, pela Equação (3.4), temos que

$$
\mathbb{E}(Y) \geq \frac{C_{1}}{\sqrt{\alpha p(n-1)}} \exp \left(\log n-p(n-1)\left(1-\alpha \log \left(\frac{e}{\alpha}\right)\right)\right)
$$

$\mathrm{e}$

$$
\mathbb{E}(Y) \leq \frac{C_{2}}{\sqrt{\alpha p(n-1)}} \exp \left(\log n-p(n-1)\left(1-\alpha \log \left(\frac{e}{\alpha}\right)\right)\right)
$$

Assim, (ii) segue aplicando a desigualdade de Markov em $Y$.

Como os graus de entrada dos vértices são variáveis aleatórias independentes, $Y$ é uma variável aleatória binomial com probabilidade $p^{\prime}$ dada pela Equação (3.4). Assim, $\operatorname{Var}(Y)=$ $n p^{\prime}\left(1-p^{\prime}\right) \leq \mathbb{E} Y$ e então, pela desigualdade de Chebyshev, temos que

$$
\operatorname{Pr}(Y=0) \leq \frac{\operatorname{Var}(Y)}{(\mathbb{E} Y)^{2}} \leq \frac{1}{\mathbb{E} Y} \leq \frac{\sqrt{\alpha p(n-1)}}{C_{1}} \exp \left(-\log n+p(n-1)\left(1-\alpha \log \left(\frac{e}{\alpha}\right)\right)\right)
$$

e provamos (iii). A prova para $\delta^{+}$é análoga.

Para qualquer $v \in[n]$, (iv) segue trivialmente da Equação (3.5) (e seu análogo para $d^{+}(v)$ ) e do fato que $d^{-}(v)$ e $d^{+}(v)$ são variáveis aleatórias independentes.

Observação 3.3.2. Observe que no Lema 3.3.1 o que determina se os limitantes das probabilidades é a escolha de $\alpha$ que influenciará na função

$$
F(\alpha)=\left(1-\alpha \log \left(\frac{e}{\alpha}\right)\right)=1-\alpha+\alpha \log (\alpha)
$$

Ademais, a função $F(x)$ é contínua e estritamente decrescente para $x \in(0,1)$ onde $F(1)=0$ e $\lim _{x \rightarrow 0^{+}} F(x)=1$.

Para todo $\phi>1$, existe um único $\alpha \in(0,1)$ tal que $F(\alpha)=1 / \phi$. Por isso é possível escolhermos $\varepsilon>0$ como na Figura 3.1 que nos dá que o grau de entrada mínimo de $\mathcal{D}(n, p)$ é próximo a $\alpha(n-1) p$ com alta probabilidade quando estamos trabalhando com $p=\phi \log n / n-1$.

Agora vamos utilizar o Lema 3.3.1 para obtermos limitantes para o grau de entrada mínimo em cada intervalo de $p=p(n) \in[0,1]$.

Corolário 3.3.3. Seja $h(n)=o(\log n)$ uma função e seja $p=p(n)=(\log (n)+h(n)) /(n-1)$. Então $\delta^{-}=o(\log n)$ a.q.c. 


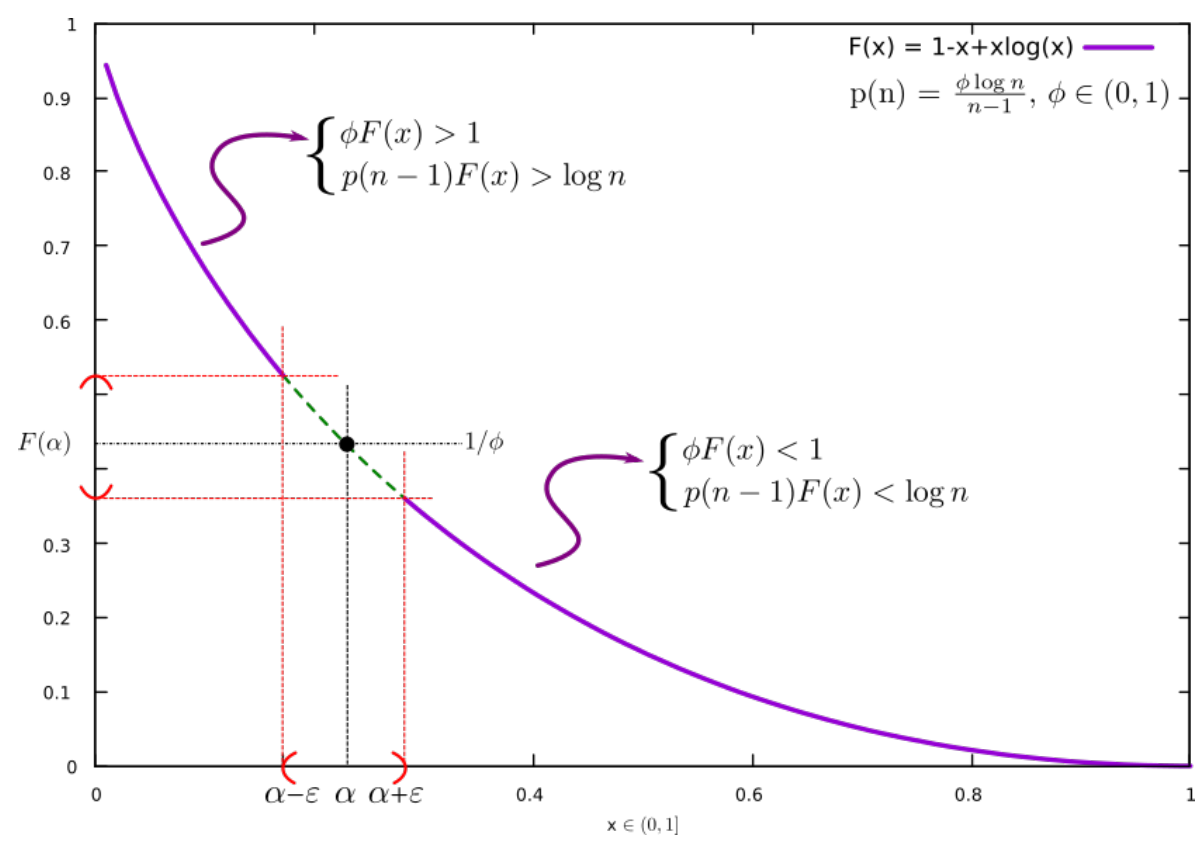

Figura 3.1: Gráfico da função $F(x)=1-x+x \log (x)$ para $x \in(0,1)$

Demonstração. É suficiente provar o resultado para $h(n) \geq 0$. Fixe $\alpha \in(0,1)$ uma constante. Observe que $F(\alpha)=1-\alpha \log (e / \alpha)<1$. O Lema 3.3.1(iii) nos diz que

$$
\operatorname{Pr}\left(\delta^{-}>\alpha p(n-1)\right) \leq \frac{\sqrt{\alpha p(n-1)}}{C_{1}} \exp (F(\alpha)(\log n+h(n))-\log n)=o(1) .
$$

Corolário 3.3.4. Seja $p=p(n)=(\log (n)+h(n)) /(n-1)$ tal que $h(n)=O(\log \log n)$. Existe uma constante $C>0$ tal que $\delta^{-} \leq C$ a.q.c.

Demonstração. Como $h(n)=O(\log \log n)$, existe uma constante $C>0$ tal que $h(n)<C \log \log n$ para $n$ suficientemente grande. Seja $Y$ a variável aleatória que determina a quantidade de vértices com grau de entrada $C$ em $\mathcal{D}(n, p)$. Desta forma, temos que

$$
\begin{aligned}
\mathbb{E}[Y] & =n\left(\begin{array}{c}
n-1 \\
C
\end{array}\right) p^{C}(1-p)^{n-C-1} \\
& \geq \exp (\log n+C \log \log n-C \log C-(n-1) p+o(1)) \\
& \geq \exp (-h(n)+C \log \log n-C \log C+o(1)) .
\end{aligned}
$$

Como o grau de entrada de vértices distintos são independentes, então pela desigualdade de Chebyshev temos $\operatorname{Pr}(Y=0)=o(1)$. Assim temos que o grau de entrada mínimo é no máximo $C$ 
a.q.c.

Para o próximo resultado, usamos a análise da função $F(x)=1-x+x \log x$ (veja a Observação 3.3.2 e a Figura 3.1).

Corolário 3.3.5. Seja $\phi>1$ uma constante. Existe uma constante $\alpha \in(0,1)$ tal que $F(\alpha)=$ $1-\alpha+\alpha \log \alpha=1 / \phi$. Para todo $p=p(n) \sim \phi \log (n) /(n-1)$, temos que $\delta^{-} \sim \alpha p(n-1)$ e $\delta^{+} \sim \alpha p(n-1)$ a.q.c.

Demonstração. Seja $\gamma=\gamma(n)$ tal que $\gamma(n)=p(n-1) / \log n$. Fixe $\varepsilon \in(0, \min \{\alpha, 1-\alpha\})$ uma constante. Observe que $\gamma \sim \phi$.

Temos que $\lim _{n \rightarrow \infty} \gamma(n) F(\alpha+\varepsilon)<F(\alpha) \lim _{n \rightarrow \infty} \gamma(n)=1$ e pelo Lema 3.3.1(iii), temos que $\delta^{-} \leq(\alpha+\varepsilon) p(n-1)$ a.q.c. Por outro lado, analogamente, temos que $\lim _{n \rightarrow \infty} \gamma(n) F(\alpha-\varepsilon)>$ $F(\alpha) \lim _{n \rightarrow \infty} \gamma(n)=1$, e, pelo Lema 3.3.1(ii), temos que $\delta^{-} \geq(\alpha-\varepsilon) p(n-1)$ a.q.c. A prova para $\delta^{+}$é análoga.

Definição 3.3.6 (Vértice leve). Dado um digrafo $D=(V, A)$, dizemos que um vértice $v$ de $D$ é $\varepsilon$-leve de entrada se $d^{-}(v) \leq \delta^{-}+\varepsilon n p$ e $\varepsilon$-leve de saída se $d^{+}(v) \leq \delta^{+}+\varepsilon n p$.

Lema 3.3.7. Seja $\varphi \geq 0.9$ uma constante. Existem constantes positivas $\varepsilon, C$ e $n_{0}$ tais que, para todo $n \geq n_{0}$ e $0.9 \log n /(n-1) \leq p \leq \varphi \log n /(n-1)$, com probabilidade pelo menos $1-n^{-C}$, não existe um par $(u, v)$ de vértices $\varepsilon$-leves de entrada tal que $u v \in A$ ou $\Gamma^{-}(v) \cap \Gamma^{-}(u) \neq \varnothing$ e não existe um par $(u, v)$ de vértices $\varepsilon$-leves de saída tal que $u v \in A$ ou $\Gamma^{+}(v) \cap \Gamma^{+}(u) \neq \varnothing$.

Demonstração. Afirmamos que é possível escolher $\alpha$ e $\varepsilon$ tais que o lado direito da inequação do Lema 3.3.1(iii) e (i) (com $\alpha$ substituído por $\alpha+\varepsilon$ ) são no máximo $B n^{-0.19}$ e $B n^{-0.7}$, respectivamente, para uma constante $B$. Isso implica que

$$
\operatorname{Pr}\left(\delta^{-}>\alpha(n-1) p\right) \leq B n^{-0.19} \text { e } \operatorname{Pr}\left(d^{-}(v) \leq(\alpha+\varepsilon) p(n-1)\right) \leq B n^{-0.7}, \forall v \in V .
$$

Seja $S$ o conjunto de vértices $v \in[n]$ tal que $d^{-}(v) \leq(\alpha+\varepsilon) p(n-1)$. Então, para quaisquer vértices $u, v \in V$, pela nossa escolha de $\alpha$ e $\varepsilon$, temos que

$$
\begin{aligned}
\operatorname{Pr}(u v \in A, u \in S \text { e } v \in S) & =p \operatorname{Pr}(v \in S \mid u v \in A) \operatorname{Pr}(u \in S \mid u v \in A) \\
& \leq(1+o(1)) p B^{2} n^{-1.4} .
\end{aligned}
$$


Como temos nos máximo $n(n-1)$ escolhas para $(u, v)$ e $p \leq \varphi \log n /(n-1)$, então o valor esperado da quantidade de pares de vértices adjacentes em $S$ é $(1+o(1)) \varphi B^{2} n^{-0.4} \log n$. Assim, a probabilidade de que existem vértices $\varepsilon$-leves de entrada adjacentes é no máximo $B n^{-0.19}+(1+o(1)) \varphi B^{2} n^{-0.4} \log n \leq(1 / 2) n^{-0.18}$ para $n$ suficientemente grande.

Para quaisquer vértices $u, v, z \in V$, pela nossa escolha de $\alpha$ e $\varepsilon$, temos que

$$
\begin{aligned}
\operatorname{Pr}(z u, z v \in A, u \in S \text { e } v \in S) & =p^{2} \operatorname{Pr}(v \in S \mid z v \in A) \operatorname{Pr}(u \in S \mid z v \in A) \\
& \leq(1+o(1)) B^{2} p^{2} n^{-1.4}
\end{aligned}
$$

Assim, como temos no máximo $n(n-1)(n-2)$ escolhas para $(u, v, z)$ e $p \leq \varphi \log n /(n-1)$, o valor esperado da quantidade de pares de vértices $\varepsilon$-leves de entrada adjacentes é $(1+o(1)) \varphi B^{2} n^{-0.4} \log ^{2} n$ e o resultado seque como acima.

Por fim, falta apresentar como escolher $\alpha$ e $\varepsilon$ para obter os limitantes desejados para as probabilidades na Equação (3.7). Sejam $F(x)=1-x+x \log x$ e $\beta$ a constante de modo que $\beta \sim(n-1) p / \log n$. Pela hipótese, $0.9 \leq \beta \leq \varphi$. Escolha $\alpha$ tal que $\beta F(\alpha)=0.8$. Então, o lado direito do Lema 3.3.1(iii) torna-se $O(1) \exp (-0.2 \log n+(1 / 2) \log \log n) \leq O\left(n^{-0.19}\right)$. Podemos então escolher $\varepsilon>0$ de modo que $\beta F(\alpha+\varepsilon)=0.7$ e o lado direito do Lema 3.3.1(i) torna-se $O(1) \exp (-\beta \log n F(\alpha+\varepsilon)-(1 / 2) \log \log n)=O(1) \exp (-0.7 \log n-(1 / 2) \log \log n)$.

O próximo resultado segue imediatamente da desigualdade de Chernoff (Teorema 2.5.3).

Lema 3.3.8. Seja $\psi=\psi(n)=\omega(1)$. Seja $p=p(n)$ tal que $p \cdot(n-1)=\psi \log n$. Então existe $0 \leq \phi=o(1)$ tal que a.q.c. para todo $v \in[v]$ temos que $(1-\phi) p(n-1) \leq d^{-}(v) \leq(1+\phi) p(n-1)$ $e(1-\phi) p(n-1) \leq d^{+}(v) \leq(1+\phi) p(n-1)$.

\subsection{Estimando $\lambda(\mathcal{D}(n, p))$}

Nesta seção, apresentamos estimativas para $\lambda=\lambda(\mathcal{D}(n, p))$ de acordo com o intervalo de $p$. Começamos provando que $\tau(D) \leq \lambda(D)$ para todo diagrafo $D$. Seja $Y_{k}(D)$ a quantidade de vértices com grau de entrada $k$ em $D$.

Lema 3.4.1. Para todo digrafo $D, \tau(D) \leq \lambda(D)$. 
Demonstração. Seja $\ell=\tau(D)$ e seja $\mathcal{P}$ a subpartição onde as partes são conjuntos unitários formados pelos vértices de grau de entrada no máximo $\ell-1$. Pela desigualdade de Frank (Teorema 3.2.4) temos que

$$
\sum_{i=1}^{\ell-1}\left(i Y_{i}(D)\right) \leq \ell\left(\sum_{i=0}^{\ell-1} Y_{i}(D)-1\right) .
$$

Ou seja, $\ell \leq \sum_{i=0}^{\ell-1}(\ell-i) Y_{i}(D)$. Pela definição de $\lambda(D)$, temos que $\lambda(D) \geq \ell=\tau(D)$.

Uma afirmação trivial, mas que será útil nas demonstrações desta seção é a seguinte.

Afirmação 3.4.2. Seja $D$ um digrafo e $k \geq 0$ um inteiro. se $Y_{k} \geq k+1$, então $\lambda(D) \leq k+1$

A seguir, usamos $Y_{k}$ para denotar $Y_{k}(\mathcal{D}(n, p))$. Para provar que $\lambda(\mathcal{D}(n, p))$ está próximo do grau de entrada mínimo precisamos definir $\delta^{*}$.

Definição 3.4.3. Denote $\delta^{*}=\delta^{*}(\mathcal{D}(n, p))$ como o menor inteiro $k \geq 0$ tal que $\mathbb{E}\left[Y_{k}\right] \geq 1$ em $\mathcal{D}(n, p)$.

O seguinte resultado mostra a relação entre $\delta^{*}$ e $\delta^{-}$.

Lema 3.4.4. Seja $p=p(n) \sim \log (n) /(n-1)$. Então $\delta^{-} \in\left\{\delta^{*}-1, \delta^{*}, \delta^{*}+1\right\}$ a.q.c. Ademais, temos que, se $\mathbb{E}\left[Y_{\delta^{*}-1}\right]=o(1)$, então $\delta^{-} \in\left\{\delta^{*}, \delta^{*}+1\right\}$ e, se $\mathbb{E}\left[Y_{\delta^{*}-1}\right]=\Omega(1)$, então $\delta^{-} \in\left\{\delta^{*}-1, \delta^{*}\right\}$.

Demonstração. Pela definição do valor esperado de $Y_{k}$ podemos observar que existe um $k^{*}(n)=$ $o(\log n)$ tal que $\mathbb{E}\left[Y_{k^{*}(n)}\right]=\omega(1)$. Então, pela definição de $\delta^{*}$, temos $\delta^{*}=o(\log n)$.

Para todo $k=o(\log n)$, temos que

$$
\mathbb{E}\left[Y_{k-1}\right] / \mathbb{E}\left[Y_{k}\right] \sim \frac{k}{(n-1) p}
$$

Primeiro, assuma que $\mathbb{E}\left[Y_{\delta^{*}-1}\right]=o(1)$. Pela desigualdade de Markov e pela Equação (3.8), temos que $\delta^{-} \geq \delta^{*}$ a.q.c.

Novamente pela Equação (3.8), temos que $\mathbb{E}\left[Y_{\delta^{*}+1}\right] \sim n p / \delta^{*} \cdot \mathbb{E}\left[Y_{\delta^{*}}\right]=\omega(1)$. Assim, pela desigualdade de Chebychev (usando a independência dos graus de entrada), temos que $\operatorname{Pr}\left(Y_{\delta^{*}+1}=0\right)=o(1)$, de modo que $\delta^{-} \leq \delta^{*}+1$ a.q.c.

A prova para o caso $\mathbb{E}\left[Y_{\delta^{*}-1}\right]=\Omega(1)$ é similar. O resultado segue então pelo princípio da subsubsequência. 
Lema 3.4.5. Seja $\phi>1$ uma constante. Se $p=p(n) \sim \phi \log (n) /(n-1)$, então $\lambda \sim \delta^{-}$.

Demonstração. Pelo Corolário 3.3.5, existe $\alpha \in(0,1)$ tal que $F(\alpha)=1-\alpha+\alpha \log (\alpha)=1 / \phi$ e temos $\delta^{-} \sim \alpha p(n-1)$ a.q.c. Fixe $\varepsilon>0$, o Lema 3.3.1(i) garante que existe $0<\beta<1$, onde $\beta$ depende de $\alpha$ e $\varepsilon$, tal que, para qualquer vértice $v$, temos

$$
\operatorname{Pr}\left(d^{-}(v) \leq \alpha(1+\varepsilon) p(n-1)\right)=\Theta\left(n^{-\beta}\right)
$$

Como os graus de entrada de distintos vértices são independentes, aplicamos a desigualdade de Chernoff (Teorema 2.5.3) à variável aleatória binomial que conta a quantidade de vértices cujo grau de entrada é no máximo $\alpha(1+\varepsilon) p(n-1)$ para concluir que existem $\Theta\left(n^{1-\beta}\right)$ tais vértices a.q.c.

Como $n^{1-\beta}=\omega(\alpha(1+\varepsilon) p(n-1))$, pela Afirmação 3.4.2, temos que $\lambda \leq \alpha(1+\varepsilon) p(n-1) \sim$ $(1+\varepsilon) \delta^{-}$a.q.c. Como $\lambda \geq \delta^{-}$vale trivialmente, nosso resultado segue.

Lema 3.4.6. Seja $p=p(n)=(\log (n)+h(n)) /(n-1)$ tal que $h(n)=O(\log \log n)$. Então $\lambda(\mathcal{D}(n, p)) \in\left\{\delta^{-}, \delta^{-}+1\right\}$ a.q.c.

Demonstração. Seja $I=\left\{\delta^{*}, \delta^{*}+1\right\}$. Primeiro, assumimos que $\mathbb{E}\left[Y_{\delta^{*}-1}\right]=o(1)$. Pelo Lema 3.4.4, temos $\delta^{-} \in I$ a.q.c. Precisamos mostrar que $\operatorname{Pr}\left(\lambda>\delta^{-}+1\right)=o(1)$.

Faça $k=\delta^{-}$e pela Afirmação 3.4.2 temos que

$$
\begin{aligned}
\operatorname{Pr}\left(\lambda>\delta^{-}+1\right) & \leq \sum_{k \in I} \operatorname{Pr}\left(Y_{k+1} \leq k+2\right)+\operatorname{Pr}\left(\delta^{-} \notin I\right) \\
& =\sum_{k \in I} \operatorname{Pr}\left(Y_{k+1} \leq k+2\right)+o(1) .
\end{aligned}
$$

Como $|I|=2$, é suficiente mostrar que $\operatorname{Pr}\left(Y_{k+1} \leq k+2\right)=o(1)$ para $k \in I$.

Pela definição de $\delta^{*}$, temos que $\mathbb{E}\left[Y_{k}\right] \geq 1$, e, pelo Corolário 3.3.4, temos que existe $C>0$ tal que $\delta^{-} \leq C$ a.q.c. Como $\delta^{-} \in I$ a.q.c., temos $k=O(1)$. Ademais, $\mathbb{E}\left[Y_{k+1}\right] \sim n p / k \cdot \mathbb{E}\left[Y_{k}\right]$, de modo que $\mathbb{E}\left[Y_{k+1}\right] \geq n p / k(1+o(1))=\omega(1)$.

Pela desigualdade de Chernoff (Teorema 2.5.3), $\operatorname{Pr}\left(Y_{k+1} \leq(1 / 2) \mathbb{E}\left[Y_{k+1}\right]\right) \leq \exp (-A \log n)$ ), onde $A>0$. Assim, $Y_{k+1}>k+2$ a.q.c. e, pela Afirmação 3.4.2, temos que $\lambda \leq \delta^{-}+1$ a.q.c.

A prova para o caso quando $\mathbb{E}\left[Y_{\delta^{*}-1}\right]=\Omega(1)$ é similar e o resultado segue pelo princípio da subsubsequência. 
Lema 3.4.7. Seja $p=p(n)=(\log (n)+h(n)) /(n-1)$ tal que $h(n)=o(\log n)$ e $h(n)=$ $\Omega(\log \log n)$. Então $\lambda(\mathcal{D}(n, p)) \sim \delta^{-}$a.q.c.

Demonstração. Fixe $\varepsilon>0$ uma constante e faça $T=\left\lfloor(1+\varepsilon) \delta^{-}\right\rfloor$. A prova é bastante similar a prova do Lema 3.4.6 e abordaremos somente o caso onde $\mathbb{E}\left[Y_{\delta^{*}-1}\right]=o(1)$.

Pelo Lema 3.4.4, temos que $\delta^{-} \in I:=\left\{\delta^{*}, \delta^{*}+1\right\}$ a.q.c. Seja $k=\delta^{-}$, pela contrapositiva da Afirmação 3.4.2, $\lambda>T$ implica que $Y_{T} \leq T+1$. Pelo Corolário 3.3.3, temos que $\delta^{-}=o(\log n)$ e, pela definição de $T$, temos que $T=o(\log n)$. Desta forma, temos que

$$
\frac{\mathbb{E}\left[Y_{T}\right]}{\mathbb{E}\left[Y_{k}\right]} \sim\left(\frac{n p}{k}\right)^{T-k}=\omega(k)
$$

Como $\mathbb{E}\left[Y_{k}\right]=\Omega(1)$, utilizando a desigualdade de Chernoff (Teorema 2.5.3), temos que $Y_{T}=$ $\omega(T)$ a.q.c., e, pela Afirmação 3.4.2, isso implica que $\lambda \leq T$ a.q.c.

O seguinte resultado segue imediatamente do Lema 3.3.8 e do fato que $\lambda$ está entre o grau de entrada mínimo e grau de entrada máximo.

Corolário 3.4.8. Seja $\phi=\phi(n)=\omega(1)$ uma função. Seja $p=p(n) \sim \phi \log (n) /(n-1)$. Temos que $\lambda(n) \sim p(n-1)$ a.q.c.

\subsection{Propriedades de expansão}

Nesta seção, investigaremos as propriedades de cortes em $\mathcal{D}(n, p)$. Procuramos boas estimativas para a quantidade de arcos que cruzam os diversos cortes para que assim possamos estimar a quantidade de arcos que entram nas partes de numa dada subpartição. Tendo boas estimativas para as subpartições conseguimos utilizar a desigualdade de Frank (Teorema 3.2.4) para obtermos limitantes inferiores para o nosso problema.

Começamos provando um resultado simples sobre a quantidade de arcos que vão de um conjunto "grande" de vértices para outro conjunto de vértices também "grande".

Lema 3.5.1. Sejam $f=f(n) \rightarrow \infty$ uma função e $\zeta$ uma constante positiva. Existem constantes positivas $n_{0}$ e $C$ tais que, para todo $n>n_{0}$ e $p=p(n) \in[f(n) / n, 1]$, a probabilidade de que existem conjuntos disjuntos $S, S^{\prime} \subseteq[n]$ com tamanhos pelo menos $\zeta n$ tais que $\left|A\left(S^{\prime}, S\right)\right|<$ $\zeta^{2} n^{2} p / 2$ é no máximo $n^{-C}$. 
Demonstração. Sejam $S, S^{\prime} \subseteq[n]$ tais que são conjuntos disjuntos com tamanho pelo menos $\zeta n$. Observe que a variável aleatória $\left|A\left(S, S^{\prime}\right)\right|$ tem distribuição $\operatorname{Bin}\left(|S|\left|S^{\prime}\right|, p\right)$. Assim, $\mathbb{E}\left(\left|A\left(S, S^{\prime}\right)\right|\right)=|S|\left|S^{\prime}\right| p$ e pela desigualdade de Chernoff (Teorema 2.5.3), temos

$$
\operatorname{Pr}\left(\left|A\left(S, S^{\prime}\right)\right| \leq \frac{\zeta^{2} n^{2} p}{2}\right) \leq \exp \left(-\frac{\zeta^{2} n^{2} p}{4}\right)
$$

Pelo limite da união, a probabilidade de que existem conjuntos disjuntos $S, S^{\prime} \subseteq[n]$ com tamanho pelo menos $\zeta n$ tais que $\left|A\left(S, S^{\prime}\right)\right|<\zeta^{2} n^{2} p / 2$ é no máximo

$$
\begin{aligned}
\sum_{s, s^{\prime} \geq \zeta n}\left(\begin{array}{l}
n \\
s
\end{array}\right)\left(\begin{array}{l}
n \\
s^{\prime}
\end{array}\right) \exp \left(-\frac{\zeta^{2} n^{2} p}{4}\right) & \leq 4^{n} \exp \left(-\frac{\zeta^{2} n^{2} p}{4}\right) \\
& <\exp \left(2 n-\frac{\zeta^{2} n^{2} p}{4}\right)
\end{aligned}
$$

e o resultado segue devido a $n p \geq f \rightarrow \infty$.

No próximo resultado, provamos um lema sobre a quantidade de arcos induzidos em conjuntos "não tão grandes". O lema será utilizado para argumentar que muitos arcos devem sair de tais conjuntos.

Lema 3.5.2. Sejam $f=f(n) \rightarrow \infty$ uma função e $\phi$ uma constante positiva. Existem constantes positivas $\zeta, n_{0}$ e $C$ tais que para todo $n>n_{0}$ e $p=p(n) \in[f / n, 1]$, a probabilidade de existir um conjunto $S \subseteq[n]$ com tamanho $|S| \leq \zeta n$ tal que $|A[S]|>\phi n|S| p$ é no máximo $e^{-C f^{2}}$.

Demonstração. Seja $\zeta>0$ suficientemente pequeno de modo que $e \zeta / \phi \leq e^{-1 / \phi^{2}}$. Seja $S \subseteq[n]$ um conjunto com tamanho $s \leq \zeta n$. Se $s \leq \phi n p$, então $|A[S]| \leq s(s-1) \leq \phi n p s$. Então assuma $s \geq \phi n p$. A probabilidade de $|A[S]|>\lceil\phi n p s\rceil$ é no máximo $\left(\begin{array}{c}s(s-1) \\ \lceil\phi n s\rceil\end{array}\right) p^{\lceil\phi n p s\rceil}$.

O valor esperado da quantidade de conjuntos $S$ com tamanho no máximo $\zeta n$ com $|A[S]|>\phi p n|S|$ é no máximo

$$
\begin{aligned}
\sum_{\phi n p \leq s \leq \zeta n}\left(\begin{array}{c}
n \\
s
\end{array}\right)\left(\begin{array}{c}
s^{2} \\
\lceil\phi n s p\rceil
\end{array}\right) p^{\lceil\phi n s p\rceil} \leq \sum_{\phi n p \leq s \leq \zeta n}\left(\frac{n e}{s}\right)^{s}\left(\frac{s e}{\phi n}\right)^{\lceil\phi n s p\rceil} \\
=\sum_{\phi n p \leq s \leq \zeta n}\left(\left(\frac{e^{2}}{\phi}\right)\left(\frac{s e}{\phi n}\right)^{\frac{\lceil\phi n s p]}{s}-1}\right)^{s} \\
\leq \sum_{\phi n p \leq s \leq \zeta n}\left(\left(\frac{e^{2}}{\phi}\right)\left(\frac{\zeta e}{\phi}\right)^{\frac{\lceil\phi n s p\rceil}{s}-1}\right)^{s} \text { Como } s \leq \zeta n \text { e } n p \geq f \rightarrow \infty
\end{aligned}
$$




$$
\begin{aligned}
& \leq \sum_{\phi n p \leq s \leq \zeta n}\left(\left(\frac{e^{2}}{\phi}\right)\left(\frac{\zeta e}{\phi}\right)^{\phi n p-1}\right)^{s} \operatorname{Como} \zeta e / \phi<1 \\
& \leq \sum_{\phi n p \leq s \leq \zeta n}\left(\beta e^{-\frac{n p}{\phi}}\right)^{s}, \quad \text { para } \beta=e / \zeta \operatorname{como} \zeta e / \phi<e^{-1 / \phi^{2}} \\
& \leq 2\left(\beta e^{-\frac{n p}{\phi}}\right)^{\phi n p} \leq e^{-C(n p)^{2}} \leq e^{-C f^{2}},
\end{aligned}
$$

para uma constante $C$ e $n$ suficientemente grande, o resultado segue pela desigualdade de Markov.

No próximo lema, relacionamos $d^{-}(S)$ e $\delta^{-}$quando $p=(1+\Omega(1)) \log n /(n-1)$ e $S$ tem tamanho entre 2 e $n-2$.

Lema 3.5.3. Seja $\psi$ uma constante positiva. Existem constantes positivas $\varepsilon$ e $n_{0}$ tais que, para $p=p(n) \in[(1+\psi) \log n /(n-1), 1]$ a probabilidade de existir um conjunto $S \subset[n]$ com $2 \leq|S| \leq n-2$ tal que $d^{-}(S)<1.5 \delta^{-}$é no máximo $n^{-\varepsilon}$.

Demonstração. Seja $\gamma(n)$ tal que $p(n-1)=\gamma(n) \log n$. Primeiro assuma que $\gamma(n) \geq 100$. Sejam $0<\varepsilon_{1}<5 / 2-\sqrt{6}, \tau=2\left(1+\varepsilon_{1}\right) / 10$ e $\varepsilon_{2}>0$ tais que $2(1-\tau)\left(1-\varepsilon_{1}\right) \geq 1.5\left(1+\varepsilon_{2}\right)$.

Sejam $S \subseteq[n]$ e $\bar{S}=[n] \backslash S$. Temos $d^{-}(S)=d^{+}(\bar{S})=|A(\bar{S}, S)|$ que é distribuído como $\operatorname{Bin}(s \bar{s}, p)$ onde $s=|S|$ e $\bar{s}=|\bar{S}|$. Então, pela desigualdade de Chernoff (Teorema 2.5.3) e pelo limite da união, a probabilidade de que existe um conjunto $S \subset[n]$ com $2 \leq|S| \leq n-2$ tal que $d^{-}(S)<(1-\tau) s \bar{s} p$ é no máximo

$$
\begin{aligned}
\sum_{2 \leq s \leq n-2}\left(\begin{array}{l}
n \\
s
\end{array}\right) \exp \left(-\frac{\tau^{2} s \bar{s} p}{2}\right) & =2 \sum_{2 \leq s \leq n / 2}\left(\begin{array}{l}
n \\
s
\end{array}\right) \exp \left(-\frac{\tau^{2} s \bar{s} p}{2}\right) \\
& \leq 2 \sum_{2 \leq s \leq n / 2} \exp \left(s+s \log n-s \log s-\frac{\tau^{2}|S||\bar{S}| p}{2}\right) \\
& \leq 2 \sum_{2 \leq s \leq n / 2} \exp \left(s \log n\left(1+\frac{1-\log s}{\log n}-\frac{\gamma \tau^{2}}{4}\right)\right) \\
& \leq 2 \sum_{2 \leq s \leq n / 2} \exp \left(-\varepsilon_{1} s \log n\right) \leq 4 n^{-2 \varepsilon_{1}} .
\end{aligned}
$$

Observe que, se $|S| \leq \varepsilon_{1} n$ ou $|\bar{S}| \leq \varepsilon_{1} n$, pelas nossas escolhas de $\tau, \varepsilon_{1}$ e $\varepsilon_{2}$,

$$
(1-\tau)|S||\bar{S}| p \geq(1-\tau) 2\left(1-\varepsilon_{1}\right) n p \geq 1.5\left(1+\varepsilon_{2}\right) n p .
$$


Se $|S| \geq \varepsilon_{1} n$ e $|\bar{S}| \geq \varepsilon_{1} n$, então, para $n$ suficientemente grande,

$$
(1-\tau)|S||\bar{S}| p \geq(1-\tau) \varepsilon_{1}^{2} n^{2} p>1.5\left(1+\varepsilon_{2}\right) n p .
$$

Então é suficiente mostrar que $\delta^{-} \leq\left(1+\varepsilon_{2}\right) n p$ com probabilidade pelo menos $1-n^{-\varepsilon}$. Isso segue imediatamente do fato que, pela desigualdade de Chernoff (Teorema 2.5.3), a quantidade de total de arcos no digrafo aleatório satisfaz $|A| \geq\left(1+\varepsilon_{2}\right) n(n-1) p$ com probabilidade no máximo $\exp \left(-\varepsilon_{2}^{2} n(n-1) p / 2\right)$.

Agora assuma que $1+\psi \leq \gamma(n) \leq 100$. Pelo Corolário 3.3.5, existem $\alpha_{1}(n), \alpha_{2}(n)$ e uma contante $x_{1}>0$ tais que

$$
x_{1}<\alpha_{1}(n)<\alpha_{2}(n)<1, \quad \alpha_{2}(n)-\alpha_{1}(n) \leq x_{1} / 7
$$

e

$$
\alpha_{1} p n \leq \delta^{-} \leq \alpha_{2} p n \quad \text { e } \quad \alpha_{1} p n \leq \delta^{+} \leq \alpha_{2} p n
$$

Sejam $\zeta>0$ e $C_{1}>0$ dadas pelo Lema 3.5.2 aplicado a $\phi=x_{1} / 7$ e $f(n)=\gamma(n) \log n$. Seja também $C_{2}$ dado pelo Lema 3.5.1 for $\zeta$ e $f(n)$.

Fixe $S \subseteq[n]$. Se $|S|,|\bar{S}| \geq \zeta n$, pelo Lema 3.5.1, $d^{-}(S)=|A(\bar{S}, S)| \geq\left(\zeta^{2} / 2\right) n^{2} p \geq 1.5 \delta^{-}$ com probabilidade pelo menos $1-n^{-C_{2}}$ para $n$ suficientemente grande. Se $2 \leq|S|<\zeta n$, pelo Lema 3.5.2, com probabilidade pelo menos $1-e^{-C_{1}(\gamma \log n)^{2}}$, para $n$ suficientemente grande,

$$
\begin{aligned}
d^{-}(S) & =\sum_{v \in S} d^{-}(v)-|A[S]| \geq \delta^{-}|S|-\phi n p|S| \\
& \geq \delta^{-}|S|-\alpha_{1} n p|S| / 7 \geq 6 \delta^{-}|S| / 7 \geq 1.5 \delta^{-}
\end{aligned}
$$

Se $|\bar{S}| \leq \zeta n$, então

$$
\begin{aligned}
d^{+}(\bar{S}) & =\sum_{v \in \bar{S}} d^{+}(v)-|A[\bar{S}]| \geq \delta^{+}|\bar{S}|-\phi n p|\bar{S}| \\
& >|\bar{S}| n p\left(\alpha_{1}-\phi\right) \geq 1.5 n p \alpha_{2} \geq 1.5 \delta^{-}
\end{aligned}
$$

devido a $2\left(\alpha_{1}-\phi\right)>1.5\left(\alpha_{1}+\phi\right) \geq 1.5 \alpha_{2}$, e o resultado segue uma vez que $d^{-}(S)=d^{+}(\bar{S})$. 
O próximo lema é útil quando precisamos aplicar a desigualdade de Frank (Teorema 3.2.4) em partições onde uma das classes é muito grande, i.e., em subpartições onde uma parte contém a fração $(1-\varepsilon)$ do conjunto de vértices.

Lema 3.5.4. Existem constantes positivas $\phi$ e $\psi$ tais que o seguinte vale. Para qualquer função $g=g(n)$ tal que $0 \leq g(n)=o(\log n)$, existem constantes $\varepsilon>0, n_{0}>0$ e $C>0$ tais que, para qualquer $p=p(n) \in[(\log (n)-g(n)) /(n-1),(\log (n)+g(n)) /(n-1)]$, com probabilidade pelo menos $1-n^{-C}$, não existe uma partição $(X, Y, Z)$ do conjunto de vértices de $\mathcal{D}(n, p)$, satisfazendo as seguintes condições:

(i) $|X| \geq(1-\varepsilon) n$,

(ii) $|Y| \geq|Z|$ ou $|Z| \leq \phi p(n-1)$,

(iii) $d^{-}(X)+d^{-}(Y) \leq \psi p(n-1)|Y|$

Demonstração. Fixe $\phi \leq 3 / 400$ e $\psi<3 / 20$. Sejam $\zeta>0, C_{1}>0$ obtidos pelo Lema 3.5.2 $\operatorname{com} f(n)=\log n-g(n)$ e $\phi$. Sejam $\varepsilon \leq \zeta$ uma constante e $(X, Y, Z)$ uma partição do conjunto de vértices de $\mathcal{D}(n, p)$ satisfazendo as condições do Lema. Pelo Lema 3.3.1(iv) com $\alpha=0.09$ e $\varphi=1.1$, temos, para $n$ suficientemente grande, com probabilidade pelo menos $1-n^{-0.02}$, que $d^{-}(v)+d^{+}(v) \geq 0.18(n-1) p$ para todo $v \in Y$. Devido ao Lema 3.5.2, temos que $|A[Y]| \leq$ $\phi(n-1) p|Y|$ e $|A[Y \cup Z]| \leq \phi(n-1) p(|Y|+|Z|)$ com probabilidade pelo menos $1-n^{-C_{1}}$.

Assim, temos que a.q.c.

$$
\begin{aligned}
d^{-}(X)+d^{-}(Y) & =\sum_{v \in Y}\left(d^{-}(v)+d^{+}(v)\right)-2|A[Y]|-|A(Y, Z)|+|A(Z, X)| . \\
& \geq 0.18(n-1) p|Y|-2 \phi(n-1) p|Y|-\max \{|A[Y \cup Z]|,|Y||Z|\} \\
& \geq 0.18(n-1) p|Y|-2 \phi(n-1) p|Y|-2 \phi n p|Y| \\
& \geq 0.15(n-1) p|Y|
\end{aligned}
$$

como desejado.

Nos próximo dois lemas, limitamos $d^{-}(S)$ para quando $p \sim \log n /(n-1)$.

Lema 3.5.5. Seja $g=g(n)$ uma função tal que $0 \leq g(n)=o(\log n)$. Existem constantes positivas $\eta>0, n_{0}>0$ e $\beta>0$ com as seguintes propriedades. Para todo $n>n_{0}$ e toda 
função $(\log n-g(n)) /(n-1) \leq p=p(n) \leq(\log n+g(n)) /(n-1)$, com probabilidade pelo menos $1-n^{-\beta}$, existem pelo menos dois vértices com grau de entrada zero ou não existe um subconjunto $S \subseteq[n]$ com tamanho $2 \leq|S| \leq \eta n$ tal que $d^{-}(S)<\max \left\{\delta^{-}+1,2 \delta^{-}\right\}$.

Demonstração. Seja $p=p(n)$ uma função como no enunciado do lema e sejam $\varepsilon>0$ e $C_{1}$ obtidas do Lema 3.3.7 $\operatorname{com} \varphi=1$.1. Pelo Corolário 3.3.3 temos $\delta^{-}=o(\log n)$ a.q.c. Dado $\eta=\zeta>0$ pelo Lema 3.5.2 aplicado a $\phi \leq \varepsilon / 16$. Suponha que o digrafo aleatório tem no máximo um vértice com grau de entrada zero e fixe $S \subseteq[n]$ com tamanho $2 \leq|S| \leq \eta n$. Pelo Lema 3.5.2, $|A[S]| \leq \varepsilon n|S| p / 16$. Denote $S_{\ell}$ como o conjunto dos vértices em $S$ que são $\varepsilon$-leves de entrada e $S_{h}=S \backslash S_{\ell}$.

Primeiro assumimos que todos os vértices em $S$ são $\varepsilon$-leves de entrada. Pelo Lema 3.3.7, com probabilidade pelo menos $1-n^{-C_{1}}$, nenhum par de vértices $\varepsilon$-leve de entrada são adjacentes. Assim $d^{-}(S) \geq|S| \delta^{-} \geq 2 \delta^{-} \geq \delta^{-}+1$ se $\delta^{-}>0$. Se $\delta^{-}=0$, pelo fato de existir apenas um vértice $v \operatorname{com} d^{-}(v)=0$, temos $d^{-}(S) \geq|S|-1 \geq 1=\max \left\{2 \delta^{-}, \delta^{-}+1\right\}$. Agora suponha que existe pelo menos um vértice $u$ em $S$ tal que não é $\varepsilon$-leve de entrada. Observe que $d^{-}(u) \geq \delta^{-}+\varepsilon n p$, o que implica que, para $|S|<\varepsilon n p / 2$, temos $d^{-}(S) \geq|A(\bar{S}, u)| \geq d^{-}(u)-|S|+1 \geq \delta^{-}+\varepsilon n p / 2 \geq 2 \delta^{-}+1$ quando $n$ grande. Então podemos assumir que $|S| \geq \varepsilon n p / 2$.

Se $\left|S_{h}\right| \geq|S| / 8$, então $d^{-}(S) \geq \sum_{v \in S_{h}} d^{-}(v)-|A[S]| \geq|S| \varepsilon n p / 8-\varepsilon n p|S| / 16 \geq \varepsilon(n-1) p / 2 \geq$ $2 \delta^{-}+1$. Assuma que $\left|S_{h}\right| \leq|S| / 8$. Assim, $\left|S_{\ell}\right| \geq 7|S| / 8$. Temos $d^{-}(S) \geq \delta^{-}\left|S_{\ell}\right|-\left|S_{h}\right|$ devido a nenhum par de vértices $\varepsilon$-leve de entrada ter um vizinho de entrada em comum a.q.c. pelo Lema 3.3.7. Se $\delta^{-}>0$, então $d^{-}(S) \geq\left|S_{\ell}\right|-\left|S_{h}\right| \geq 3 \varepsilon(n-1) p / 8 \geq 2 \delta^{-}+1$. Assuma que $\delta^{-}=0$. Como existe um vértice com grau de entrada zero, então $d^{-}(S) \geq\left|S_{\ell}\right|-1-\left|S_{h}\right| \geq \varepsilon(n-1) p / 4 \geq$ $2 \delta^{-}+1$.

Lema 3.5.6. Seja $\phi>0$ uma constante e $g=g(n)$ uma função tal que $0 \leq g(n)=o(\log n)$. Existem constantes positivas $\eta>0, n_{0}>0$ e $\beta>0$ tais que o seguinte vale para todo $n>n_{0}$ e para toda função $(\log n-g(n)) /(n-1) \leq p=p(n) \leq(\log n+g(n)) /(n-1)$. Com probabilidade pelo menos $1-n^{-\beta}$, existem dois vértices com grau de entrada zero ou não existe um conjunto $S \subseteq[n]$ com tamanho $\phi \log n \leq|S| \leq \eta n$ tal que $d^{+}(S)<2 \delta^{-}+1$.

Demonstração. Sejam $\phi>0$ uma constante e $p=(\log (n)+h(n)) /(n-1)$ tal que $|h(n)| \leq g(n)$. Sejam $\varepsilon>0$ e $C_{1}$ obtidas através do Lema 3.3.7 $\operatorname{com} \varphi=1.1$. Podemos assumir que $\phi<\varepsilon / 16$. 
Pelo Corolário 3.3.3 temos $\delta^{-}=o(\log n)$ a.q.c. Sejam $\eta=\zeta>0$ e $C_{2}$ do Lema 3.5.2 aplicado a $\phi$ e faça $\psi \leq \phi / 2$.

Se $h(n)<-\log (\psi \log n)$, pela desigualdades de Chernoff (Teorema 2.5.3) temos que pelo menos dois vértices tem grau de entrada zero a.q.c. Então consideramos o caso $h(n) \geq-\log (\psi \log n)$.

Assuma que no máximo um vértice tem grau de entrada zero e fixe $S \subseteq[n]$ com tamanho $\phi \log n \leq|S| \leq \eta n$. Pelo Lema 3.5.2, $|A[S]| \leq \varepsilon n|S| p / 16$ com probabilidade pelo menos $1-n^{-C_{2}}$. Denote $S_{\ell}$ como o conjunto dos vértices de $S$ que são $\varepsilon$-leve de saída e $S_{h}=S \backslash S_{\ell}$. Se $\left|S_{h}\right| \geq$ $|S| / 8$, então $d^{+}(S) \geq \sum_{v \in S_{h}} d^{+}(v)-|A[S]| \geq \varepsilon n p|S| / 8-\varepsilon n p|S| / 16 \geq 2 \delta^{-}+1$. Então assuma $\left|S_{h}\right| \leq|S| / 8$, e assim $\left|S_{\ell}\right| \geq 7|S| / 8$. Então $d^{+}(S) \geq \delta^{+}\left|S_{\ell}\right|-\left|S_{h}\right|$ devido a não existir um par de vértices $\varepsilon$-leve de saída com um vértice de saída em comum. Podemos assumir que $\delta^{+}=0$. De fato, se $\delta^{+}>0$, então $d^{+}(S) \geq\left|S_{\ell}\right|-\left|S_{h}\right| \geq(3 / 8) \varepsilon(n-1) p \geq 2 \delta^{-}+1$.

Agora vamos estimar a quantidade de vértices com grau de saída zero. O valor esperado da quantidade de vértices com grau zero é $n(1-p)^{n-1}=\exp (-h(n)+o(1))$. É claro que quanto menor $h(n)$, maior a quantidade de vértices com grau de saída zero. Se $h(n)=-\log (\psi \log n)$, então o valor esperado da quantidade de vértices com grau de saída igual a zero é igual a $\psi \log n(1+o(1))$. Assim, pela desigualdade de Chernoff (Teorema 2.5.3), existe um $\beta>0$ tal que a probabilidade que existe mais do que $5 \phi \log n / 8$ vértices com grau de saída zero é no máximo $\exp (-\beta \log n)$. Então, $d^{+}(S) \geq\left|S_{\ell}\right|-(5 \phi / 8) \log n-|S| / 8 \geq 3|S| / 4-5 \phi \log n / 8 \geq 2 \delta^{-}+1$ para/ $h(n) \geq-\log (\psi \log n)$.

\subsection{Prova do resultado principal}

Para a prova do Teorema 3.2.3, precisamos considerar cinco diferentes regimes de probabilidade, os quais são descritos no seguinte resultado.

Lema 3.6.1. Seja $p=p(n) \in[0,1]$. Se

(i) $p \leq(\log (n)-g(n)) /(n-1)$, para uma função $g(n)=\omega(1)$; ou

(ii) $(\log (n)-g(n)) /(n-1) \leq p \leq(\log (n)+g(n)) /(n-1)$, para uma função $g(n)=\omega(1)$ e $g(n)=O(\log \log n) ;$ ou

(iii) $(\log (n)-g(n)) /(n-1) \leq p \leq(\log (n)+g(n)) /(n-1)$, para uma função $g(n)=\Omega(\log \log n)$ e $g(n)=o(\log n) ; o u$ 
(iv) $p \sim(1+\psi) \log (n) /(n-1)$, para uma constante $\psi>0$; ou

(v) $p=g(n) \log (n) /(n-1)$, para uma função $g(n)=\omega(1)$,

então $\tau(\mathcal{D}(n, p))=\lambda(\mathcal{D}(n, p))$ a.q.c.

Mostraremos agora como o Teorema 3.2.3 segue do Lema 3.6.1 acima. Seja $p=p(n) \in$ $[0,1]$. Seja $A_{n}$ o evento que $\tau(\mathcal{D}(n, p))=\lambda(\mathcal{D}(n, p))$ e $\bar{A}_{n}$ o complemento de $A_{n}$. Mostraremos que $\lim _{n \rightarrow \infty} \operatorname{Pr}\left(\bar{A}_{n}\right)=0$. Usaremos o princípio da subsubsequência. Assim, seja $\left(n_{i}\right)_{i \in \mathbb{N}}$ uma sequência crescente arbitrária onde $n_{i} \in \mathbb{N}$ para todo $i \in \mathbb{N}$. É suficiente mostrar que existe uma subsequência $\left(m_{i}\right)_{i \in \mathbb{N}}$ de $\left(n_{i}\right)_{i \in \mathbb{N}}$ tal que $\lim _{i \rightarrow \infty} \operatorname{Pr}\left(\bar{A}_{m_{i}}\right)=0$.

Seja $h(n)=(n-1) p / \log n$. Observe que $h(n) \geq 0$. Então existe uma subsequência de $\left(m_{i}\right)_{i \in \mathbb{N}}$ de $\left(n_{i}\right)_{i \in \mathbb{N}}$ tal que $\lim _{i \rightarrow \infty} h\left(n_{i}\right)=c$, onde $c$ é uma constante não negativa ou $\infty$. Se $c<1$, temos que $\lim _{i \rightarrow \infty} \operatorname{Pr}\left(\bar{A}_{m_{i}}\right)=0$ pelo Lema 3.6.1(i). Se $c=1$, temos que $\lim _{i \rightarrow \infty} \operatorname{Pr}\left(\bar{A}_{m_{i}}\right)=0$ pelo Lema 3.6.1(ii) e (iii). Se $1<c<\infty$, temos que $\lim _{i \rightarrow \infty} \operatorname{Pr}\left(\bar{A}_{m_{i}}\right)=0$ pelo Lema 3.6.1(iv). Se $c=\infty$, temos que $\lim _{i \rightarrow \infty} \operatorname{Pr}\left(\bar{A}_{m_{i}}\right)=0$ pelo Lema 3.6.1(v). Assim, podemos aplicar o princípio da subsubsequência e concluir que $\operatorname{Pr}\left(A_{n}\right)=o(1)$.

Agora vamos juntar os resultados das seções anteriores para provar o Lema 3.6.1.

Prova do Lema 3.6.1. Caso (i): Suponha que $p \leq(\log (n)-g(n)) /(n-1)$, para uma função $g(n)=\omega(1)$. Se temos dois vértices com grau de entrada 0 , então $\tau=\lambda=0$. O valor esperado da quantidade de vértices com grau de entrada zero é $n(1-p)^{n-1} \geq \exp (g(n)+o(1)) \rightarrow \infty$. Então, pela desigualdade de Chernoff (Teorema 2.5.3), existem pelo menos dois vértices com grau de entrada zero a.q.c.

Para os casos remanescentes usaremos, por simplicidade, uma partição em vez de uma subpartição. Para tal, basta fazermos $V_{0}$ o conjunto dos vértices que não estão na subpartição dada. Seja $\mathcal{P}=\left(V_{0}, \ldots, V_{t}\right)$ uma partição de $[n]$. Precisamos mostrar que $\sum_{i=1}^{t} d^{-}\left(V_{i}\right) \geq \lambda(t-1)$, uma vez que isso implica que $\tau(\mathcal{D}(n, p)) \geq \lambda(\mathcal{D}(n, p))$ pelo Teorema 3.2.4.

Casos (ii) e (iii): Sejam $\phi>0$ e $\varepsilon>0$ obtidas pelo Lema 3.5.4. Fixe $\eta_{1}>0$ como no Lema 3.5.5 e seja $\eta_{2}>0$ obtida pela aplicação do Lema 3.5.6 para $\phi$. Seja $\alpha=\min \left\{\varepsilon, \eta_{1}, \eta_{2}\right\}$. Podemos assumir que a quantidade de vértices com grau de entrada zero é no máximo um, pois caso contrário $\tau=\lambda=0$ trivialmente.

Primeiro suponha que existe $j>0$ tal que $\left|V_{j}\right| \geq(1-\alpha) n$. Seja $B=\bigcup_{i>0, i \neq j} V_{i}$. Observe que o resultado é trivial se $B=\emptyset$, pois $t-1=0$ nesse caso. Assim suponha $|B|>0$. Se $\left|V_{0}\right| \leq \phi n p$ 
ou $\left|V_{0}\right| \leq|B|$, então $d^{-}\left(V_{j}\right)+d^{-}(B)=\Theta\left(p(n-1)|B|\right.$ pelo Lema 3.5.4. Como $\lambda=O\left(\delta^{-}\right)$pelos Lemas 3.4.6 e 3.4.7 e $\delta^{-}=o(\log n)$ pelo Corolário 3.3.3, temos $d^{-}\left(V_{j}\right)+d^{-}(B)=\omega(\lambda|B|)$, de modo que

$$
\sum_{i=1}^{t} d^{-}\left(V_{i}\right) \geq d^{-}\left(V_{j}\right)+d^{-}(B) \geq \lambda|B| \geq \lambda(t-1) .
$$

Então assuma que $\left|V_{0}\right|>\phi n p$ e $\left|V_{0}\right|>|B| \geq 1$. Seja $I=\left\{i>0:\left|V_{i}\right|=1, d^{-}\left(V_{i}\right) \leq \lambda\right\}$. Observe que $d^{-}\left(V_{j}\right)=d^{+}\left(V_{0} \cup B\right)$, pelo Lema 3.5.6 temos $d^{-}\left(V_{j}\right) \geq d^{+}\left(V_{0} \cup B\right) \geq 2 \delta^{-}+1$ e pelos Lemas 3.4.6 e 3.4.7 temos que $2 \delta^{-}+1 \geq \lambda$ a.q.c. Ademais, para todo $V_{i} \in \mathcal{P} \backslash I$ para $i \neq j$, pelo Lema 3.5.5, $d^{-}\left(V_{i}\right) \geq \max \left\{\delta^{-}+1,2 \delta^{-}\right\}$, que é pelo menos $\lambda$ a.q.c. Seja $V(I)=\bigcup_{i \in I} V_{i}$. Então, temos

$$
\sum_{i=1}^{t} d^{-}\left(V_{i}\right) \geq \lambda(t-|I|)+\sum_{i \in I} d^{-}\left(V_{i}\right)=t \lambda-\sum_{v \in V(I)}\left(\lambda-d^{-}(v)\right) \geq t \lambda-\lambda,
$$

pela definição de $\lambda$.

Agora suponha que $\left|V_{i}\right| \leq(1-\alpha) n$ para todo $i>0$. Se $2 \leq\left|V_{i}\right| \leq \alpha n$, então $d^{-}\left(V_{i}\right) \geq$ $\max \left\{\delta^{-}+1,2 \delta^{-}\right\}$pelo Lema 3.5.5 e $d^{-}\left(V_{i}\right) \geq \lambda$ a.q.c. pelos Lemas 3.4.6 e 3.4.7. Se $\left|V_{i}\right| \geq \alpha n$, temos $\left|\bar{V}_{i}\right| \geq(1-\alpha) n$ e então, pelo Lema 3.5.1, $d^{-}\left(V_{i}\right)=\left|A\left(\bar{V}_{i}, V_{i}\right)\right| \geq \zeta^{2} n^{2} p / 2 \geq \lambda$ para $\zeta=\min \{\alpha, 1-\alpha\}$. Assim, a.q.c.

$$
\sum_{i=1}^{t} d^{-}\left(V_{i}\right) \geq \lambda(t-|I|)+\sum_{i \in I} d^{-}\left(V_{i}\right) \geq t \lambda-\lambda
$$

novamente pela definição de $\lambda$.

Caso (iv): Pelo Lema 3.5.3, todo conjunto $S \subseteq[n]$ com tamanho em $[2, n-2]$ tem $d^{-}(S) \geq 1.5 \delta^{-}$ a.q.c. Pelo Lema 3.4.5 temos $\lambda \sim \delta^{-}$a.q.c., o que implica que $d^{-}(S) \geq \lambda$. Seja $I=\left\{i>0:\left|V_{i}\right|=\right.$ $\left.1, d^{-}\left(V_{i}\right) \leq \lambda-1\right\}$. Se $\left|V_{i}\right| \leq n-2$ para todo $i$, então a.q.c. $d^{-}\left(V_{i}\right) \geq \lambda$ para todo $i \notin I$ e então

$$
\sum_{i=1}^{t} d^{-}\left(V_{i}\right) \geq \lambda(t-|I|)+\sum_{i \in I} d^{-}\left(V_{i}\right) \geq t \lambda-\lambda
$$

pela definição de $\lambda$.

Agora suponha, sem perda de generalidade, que $\left|V_{1}\right|=n-1$ (o caso $\left|V_{1}\right|=n$ é trivial). Então existe um único vértice $v \notin V_{1}$. Podemos assumir que $t=2$ devido ao caso $t=1$ ser trivial. Assim, $V_{2}=\{v\}$ e $d^{-}\left(V_{1}\right)+d^{-}\left(V_{2}\right)=d^{-}(v)+d^{+}(v) \geq \delta^{-}+\delta^{+}$. Pelo Corolário 3.3.5 
temos $\delta^{-}+\delta^{+} \geq 1.5 \delta^{-}$a.q.c. pelo Lema 3.4.5, concluímos que $d^{-}\left(V_{1}\right)+d^{-}\left(V_{2}\right) \geq 1.5 \delta^{-} \geq \lambda$ a.q.c.

Caso (v): Podemos proceder como no caso anterior, já que, para todo $S \subseteq[n]$ com tamanho em $[2, n-2]$, novamente temos $\delta^{-} \sim \lambda$ e $d^{-}(S) \geq 1.5 \delta^{-} \geq \lambda$ a.q.c. (devido a $\delta^{-} \sim(n-1) p$ a.q.c. pelo Lema 3.3.8 e $\lambda \sim(n-1) p$ a.q.c. pelo Corolário 3.4.8).

Isto nos leva ao resultado desejado como os argumentos acima se $\left|V_{i}\right| \leq n-2$ para todo $i$. Caso contrário, usamos o Lema 3.3 .8 para mostrar que $\delta^{-}+\delta^{+} \geq 1.5 \delta^{-}$a.q.c., e podemos novamente repetir a análise do caso anterior. 


\section{Capítulo 4}

\section{Densidade máxima de subtorneios}

\section{fortemente conexos}

Neste capítulo, apresentamos os resultados obtidos sobre a densidade máxima de subtorneios utilizando o método semidefinido de teoria de álgebra de flags e técnicas para extrair informações sobre a estrutura dos torneios extremais. Tais resultados foram obtidos em conjunto com Leonardo N. Coregliano e Cristiane M. Sato.

\subsection{Introdução}

Em 1964, Colombo [Col64] provou que a quantidade máxima de circuitos orientados de tamanho 4 em um torneio de tamanho ímpar é atingida pelo torneio carrossel $R_{2 n+1}$, que é definido por $V\left(R_{2 n+1}\right)=\mathbb{Z}_{2 n+1}=\{0,1, \ldots, 2 n\}$ e $A\left(R_{2 n+1}\right)=\{(v,(v+i) \bmod (2 n+1)): v \in$ $V\left(R_{2 n+1}\right)$ e $\left.i \in[n]\right\}$, onde $[n]=\{1,2, \ldots, n\}$. (Veja a Figura 2.1 para exemplos.) Dado um inteiro $k$, Beineke e Harary [BH65] estenderam esse resultado provando que o torneio carrossel $R_{n}$ maximiza a quantidade de subtorneios fortemente conexos com tamanho $k$.

O problema de maximizar o número de ocorrências de um subtorneio fixo em torneios de tamanho $n$ é, em geral, um problema difícil, e determinar quais são seus torneios extremais é ainda mais difícil. Entretanto, em vários casos, o problema de maximizar a densidade assintótica é mais fácil e solucionável, ou seja, não somente conseguimos descobrir o valor máximo da densidade assintótica, mas também conseguimos caracterizar a família extremal dos torneios.

Tal caracterização é obtida através da convergência de $\left(T_{n}\right)_{n \in \mathbb{N}}$ a um certo objeto limite $\phi$, 
i.e., para todo torneio fixo $T$, temos

$$
\lim _{n \rightarrow \infty} p\left(T ; T_{n}\right)=\phi(T)
$$

onde $p\left(T ; T_{n}\right)$ denota a densidade não rotulada de $T$ em $T_{n}$ (que é um número em $\left.[0,1]\right)^{1}$.

Existem basicamente duas abordagens para definir objetos limites. A primeira abordagem é definir o objeto limite sendo semanticamente próximo ao objeto subjacente de acordo com algumas métricas que não iremos abordar no presente trabalho. Essa abordagem tem sido executada com sucesso para várias estruturas combinatórias, tais como grafos [LS06], hipergrafos uniformes [ES12], digrafos [DJ08, Section 9] ${ }^{2}$ e permutações [HKM ${ }^{+}$13]. A segunda abordagem define o objeto limite sintaticamente, ou seja, é o estudo de que tipo de propriedades que temos em $(\phi(T))_{T \in \mathcal{T}}$ para toda sequência $\left(T_{n}\right)_{n \in \mathbb{N}}$ que satisfaz $\forall T, \lim _{n \rightarrow \infty} p\left(T ; T_{n}\right)=\phi(T)$. Essa última abordagem é precisamente o que impulsiona a teoria de álgebra de flags desenvolvida por Razborov [Raz07] e é a abordagem que usamos.

Neste capítulo, estudamos o problema de maximizar a densidade assintótica de um único torneio fortemente conexo fixo $T$ de tamanho 5, i.e., estamos interessado em computar

$$
\lim _{n \rightarrow \infty} \max \left\{p\left(T ; T_{n}\right):\left|V\left(T_{n}\right)\right|=n\right\}
$$

para $T \in\left\{T_{5}^{7}, T_{5}^{8}, T_{5}^{9}, T_{5}^{10}, T_{5}^{11}, T_{5}^{12}\right\}$ (veja a Figura 4.1$)^{3}$ e caracterizar as sequências $\left(T_{n}\right)_{n \in \mathbb{N}}$ que atingem o máximo para $\lim _{n \rightarrow \infty} p\left(T ; T_{n}\right)$. Observe que isso difere do resultado de Beineke e Harary [BH65] onde provaram que para todo $k \in \mathbb{N}$, temos

$$
\max \left\{\sum_{T \in \mathcal{S}_{k}} p\left(T ; T_{2 n+1}\right):\left|V\left(T_{2 n+1}\right)\right|=2 n+1\right\}=\sum_{T \in \mathcal{S}_{k}} p\left(T ; R_{2 n+1}\right)
$$

onde $\mathcal{S}_{k}$ denota o conjunto de todos os torneios fortemente conexos de tamanho $k$.

Organização do capítulo. O presente capítulo está organizado como segue: Na Seção 4.2, apresentamos nossos resultados referentes a torneios fortemente conexos. Na Seção 4.3, apre-

\footnotetext{
${ }^{1}$ É claro que se $\left(T_{n}\right)_{n \in \mathbb{N}}$ maximiza a densidade assintótica de $T$, então sabemos o valor de $\lim _{n \rightarrow \infty} p\left(T ; T_{n}\right)$, mas a robustez dessa caracterização é que a extremalidade para $T$ força que a densidade de outros torneios $T^{\prime} \neq T$ convirjam para outros valores específicos.

${ }^{2}$ Como esperado, o objeto limite para um torneio é um caso especial do objeto limite para digrafos.

${ }^{3}$ É fácil ver que a sequência dos valores máximos tem cauda decrescente, consequentemente é convergente.
} 


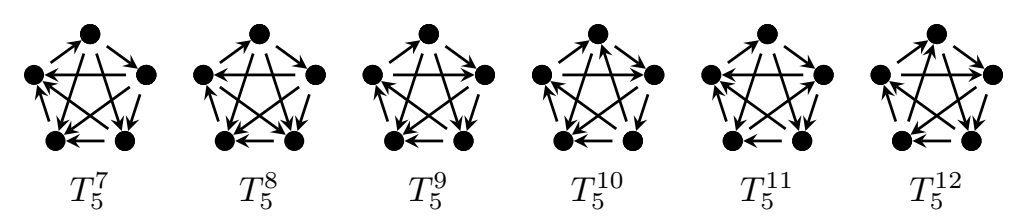

Figura 4.1: Torneios fortemente conexo de tamanho 5.

sentamos a teoria de álgebra de flags bem como seu método semidefinido e como obtermos informações das estruturas dos torneios extremais. Na Seção 4.4, apresentamos os limitantes inferiores para os nossos resultados. Na Seção 4.5, provamos o limitantes superiores utilizando o método semidefinido da teoria de álgebra de flag apresentado na Subseção 4.3.2. Por último, na Seção 4.6, apresentamos as provas para as famílias extremais, introduzimos as propriedades quase-triangulares e provamos suas relações com famílias extremais de alguns subtorneios.

\subsection{Resultados}

Dizemos que um torneio $T$ é balanceado quando todo vértice tem grau de entrada e igual ao grau saída. Para o estudo assintótico de torneios, precisamos de uma noção mais fraca de torneio balanceado. Uma sequência de torneios $\left(T_{n}\right)_{n \in \mathbb{N}}$ de tamanho crescente é assintoticamente balanceada se pelo menos $(1-o(1))\left|T_{n}\right|$ vértice de $T_{n}$ tem grau de saída $(1 / 2+o(1))\left|T_{n}\right|$.

Na teoria de álgebra de flags, a noção de objeto limite é capturada pelos homomorfismos positivos $\phi \in \operatorname{Hom}^{+}\left(\mathcal{A}^{0}, \mathbb{R}\right)$ (definidos na Seção 4.3.1). Dizemos que um homomorfismo positivo é balanceado quando é o objeto limite de uma sequência de torneios assintoticamente balanceados. Neste trabalho, provamos a extremalidade envolvendo três importantes homomorfismos balanceados como veremos a seguir.

O primeiro homomorfismo é o homomorfismo quase-aleatório $\phi_{\mathrm{qr}}$, que é o principal objeto de estudo da teoria do torneios quase-aleatórios. Definimos o homomorfismo quase-aleatório como

$$
\forall T \text { torneio, } \quad \phi_{\mathrm{qr}}(T)=\frac{|T| !}{|\operatorname{Aut}(T)| 2^{\left(\frac{|T|}{2}\right)}} .
$$

Para todo $n \in \mathbb{N}$, seja $\boldsymbol{R}_{\boldsymbol{n}, \mathbf{1 / 2}}$ o torneio aleatório de tamanho $n$ onde cada orientação de um arco é apresentada com probabilidade $1 / 2$ independentemente para todos os pares de vértices. 
Como vimos na Seção 2.4, temos que

$$
\forall T \text { torneio, } \lim _{n \rightarrow \infty} p\left(T ; \boldsymbol{R}_{\boldsymbol{n}, \mathbf{1 / 2}}\right)=\mathbb{E}\left[p\left(T ; \boldsymbol{R}_{|\boldsymbol{T}| \mathbf{1} / \mathbf{2}}\right)\right]=\phi_{\mathrm{qr}}(T)
$$

quase certamente. Assim, temos que $\lim _{n \rightarrow \infty} p\left(T_{;} R_{n, 1 / 2}\right)$ quase certamente terá como objeto limite o homomorfismo quase-aleatório $\phi_{\mathrm{qr}}$.

A teoria da quase-aleatoriedade foi iniciada com o estudo de grafos quase-aleatórios pelos artigos de Thomason [Tho87] e Chung, Graham e Wilson [CGW88] e atualmente existem ramos em diversas outras teorias como hipergrafos uniformes [CG90, Chu12, BR13], orientações de grafos [Gri13], permutações [Coo04, KP13] e torneios [CG91, KS13, Cor15].

Não apresentaremos uma revisão detalhada da teoria de quase-aleatoriedade (veja a resenha [KS06] para detalhes). A essência das propriedades de quase-aleatoriedade no contexto de homomorfismos positivos é que existem propriedades de homomorfismos $\phi \in \operatorname{Hom}^{+}\left(\mathcal{A}^{0}, \mathbb{R}\right)$ que não parecem ter relação entre si, mas que na verdade são equivalentes e forçam $\phi=\phi_{\mathrm{qr}}$. Um exemplo de uma propriedade de quase-aleatoriedade foi provada por Coregliano e Razborov [CR16]: Para todo $k \geq 4$, se $\phi$ minimiza a densidade de torneios transitivos de tamanho 4 , então $\phi=\phi_{\mathrm{qr}}$.

A respeito de $\phi_{\mathrm{qr}}$, provamos o seguinte resultado.

Teorema 4.2.1 (Coregliano-Parente-Sato[CPS15]). Temos

$$
\lim _{n \rightarrow \infty} \max \left\{p\left(T_{5}^{8} ; T_{n}\right):\left|T_{n}\right|=n\right\}=\frac{15}{128} .
$$

Ademais, uma sequência de torneios $\left(T_{n}\right)_{n \in \mathbb{N}}$ de tamanho crescente é extremal para $T_{5}^{8}$ se, e somente se, é quase-aleatória, i.e., $\left(T_{n}\right)_{n \in \mathbb{N}}$ converge para $\phi_{\mathrm{qr}}$.

O segundo homomorfismo que estudamos é o homomorfismo carrossel $\phi_{\mathrm{R}}$, que é o objeto limite da sequência $\left(R_{2 n+1}\right)_{n \in \mathbb{N}}$ de torneios carrossel. Analogamente às propriedades quasealeatórias, as propriedades quase-carrossel [Cor15] são propriedades de um homomorfismo $\phi \in$ $\operatorname{Hom}^{+}\left(\mathcal{A}^{0}, \mathbb{R}\right)$ que forçam $\phi=\phi_{\mathrm{R}}$.

No presente trabalho, provamos o seguinte resultado envolvendo $\phi_{\mathrm{R}}$. 
Teorema 4.2.2 (Coregliano-Parente-Sato[CPS15]). Temos

$$
\begin{aligned}
& \lim _{n \rightarrow \infty} \max \left\{p\left(T_{5}^{7} ; T_{n}\right):\left|T_{n}\right|=n\right\}=\frac{5}{16} \\
& \lim _{n \rightarrow \infty} \max \left\{p\left(T_{5}^{12} ; T_{n}\right):\left|T_{n}\right|=n\right\}=\frac{1}{16} .
\end{aligned}
$$

Ademais, uma sequência de torneios $\left(T_{n}\right)_{n \in \mathbb{N}}$ de tamanho crescente é extremal para $T_{5}^{7}$ ou $T_{5}^{12}$ se, e somente se, é quase-carrossel, i.e., $\left(T_{n}\right)_{n \in \mathbb{N}}$ converge para $\phi_{\mathrm{R}}$

Finalmente, o último homomorfismo estudado é o que chamamos de homomorfismo triangular $\phi_{\vec{C}_{3}}$. Definimos $\phi_{\vec{C}_{3}}$ como o objeto limite da sequência $\left(\vec{C}_{n}^{3}\right)_{n \in \mathbb{N}} \cdot{ }^{4}$ Informalmente, $\vec{C}_{n}^{3}$ é o "blow-up" recursivo do circuito direcionado de tamanho 3 (veja a definição na Seção 2.3).

Nosso resultado final de extremalidade se refere a $\phi_{\vec{C}_{3}}$.

Teorema 4.2.3 (Coregliano-Parente-Sato[CPS15]). Temos

$$
\begin{aligned}
& \lim _{n \rightarrow \infty} \max \left\{p\left(T_{5}^{9} ; T_{n}\right):\left|T_{n}\right|=n\right\}=\frac{3}{8} \\
& \lim _{n \rightarrow \infty} \max \left\{p\left(T_{5}^{11} ; T_{n}\right):\left|T_{n}\right|=n\right\}=\frac{1}{16} .
\end{aligned}
$$

Ademais, uma sequência de torneios $\left(T_{n}\right)_{n \in \mathbb{N}}$ de tamanho crescente é extremal para $T_{5}^{9}$ ou $T_{5}^{11}$ se, e somente se, é quase-triangular, i.e., $\left(T_{n}\right)_{n \in \mathbb{N}}$ converge para $\phi_{\vec{C}_{3}}$.

Motivados pela definição de $\vec{C}_{n}^{3}$, dizemos que um torneio é $\vec{C}_{3}$-decomponível (veja a Definição 4.6.8) se esse tem a mesma estrutura de $\vec{C}_{n}^{3}$ mas sem a restrição de que as partes tenham tamanhos tão iguais quanto possível. Como resultado auxiliar, provamos (Teorema 4.6.10) que um torneio é $\vec{C}_{3}$-decomponível se, e somente se, este não contém cópias de $T_{5}^{8}, T_{5}^{10}$ nem de $T_{5}^{12}$. Estendemos essa notação para homomorfismos e dizemos que o homomorfismo $\phi \in \operatorname{Hom}^{+}\left(\mathcal{A}^{0}, \mathbb{R}\right)$ é $\vec{C}_{3}$-decomponível se $\phi\left(T_{5}^{8}+T_{5}^{10}+T_{5}^{12}\right)=0$.

Como subproduto do Teorema 4.2.3, provamos algumas propriedades quase-triangulares, ou seja, propriedades que forçam um homomorfismo $\phi \in \operatorname{Hom}^{+}\left(\mathcal{A}^{0}, \mathbb{R}\right)$ a ser $\phi_{\vec{C}_{3}}$. Uma delas, a saber $L_{4}$, afirma que $\phi_{\vec{C}_{3}}$ é o único homomorfismo que é balanceado e $\vec{C}_{3}$-decomponível.

Finalizamos observando que nossos resultados falham em cobrir somente um torneio fortemente conexo com tamanho 5, a saber $T_{5}^{10}$ na Figura 4.1.

\footnotetext{
${ }^{4}$ Veja a Proposição 4.6.22 para a convergência desta sequência
} 


\section{3 Álgebra de flags}

Nesta seção, revisaremos brevemente os conceitos básicos da teoria de álgebra de flags, o seu método semidefinido e como obter informações sobre as famílias extremais. Embora nosso trabalho só lide com a teoria dos torneios, vale ressaltar que a teoria de álgebra de flags pode ser definida de forma geral para qualquer teoria universal de primeira ordem. Veja [Raz07] e [Raz10], e também as resenhas [Raz13a] e [Raz13b].

\subsubsection{Definições básicas e propriedades}

Seja $\mathcal{T}_{n}$ o conjunto de todos os torneios com $n$ vértices a menos de isomorfismos. Definimos $\mathcal{T}=\bigcup_{n \in \mathbb{N}} \mathcal{T}_{n}$ como o conjunto de todos os torneios a menos de isomorfismos com número finito de vértices. Para todo torneio $T$, denotamos o seu tamanho por $|T|=|V(T)|$.

Um tipo é um torneio com conjunto de vértices $[k]=\{1,2, \ldots, k\}$ para algum $k \in \mathbb{N}$. Dado um tipo $\sigma$ de tamanho $k=|\sigma|$, uma $\sigma$-flag é um torneio parcialmente rotulado de modo que a parte rotulada é uma cópia de $\sigma$. Formalmente, uma $\sigma$-flag é um par $(T, \theta)$, onde $T$ é um torneio e $\theta:[k] \rightarrow V(T)$ é uma imersão de $\sigma$ em $T$, ou seja, a função $\theta$ é um isomorfismo entre $\sigma$ e o torneio induzido por $\operatorname{im}(\theta)$ em $T$. Definimos o tamanho da $\sigma$-flag $F=(T, \theta)$ como $|F|=|T|$.

Estendemos a notação de isomorfismo para as $\sigma$-flags declarando que uma função $f: V\left(T_{1}\right) \rightarrow$ $V\left(T_{2}\right)$ é um isomorfismo entre flags $F_{1}=\left(T_{1}, \theta_{1}\right)$ e $F_{2}=\left(T_{2}, \theta_{2}\right)$ se $f$ é um isomorfismo entre $T_{1}$ e $T_{2}$, e $f \circ \theta_{1}=\theta_{2}$ (i.e., a função $f$ preserva rótulos). Naturalmente, dizemos que duas flags $F_{1}$ e $F_{2}$ são isomorfas (denotamos $F_{1} \cong F_{2}$ ) se existe um isomorfismo entre elas.

Isso nos permite definir $\mathcal{F}_{n}^{\sigma}$ como o conjunto de todas as $\sigma$-flags de tamanho $n$ a menos de isomorfismo e $\mathcal{F}=\bigcup_{n \in \mathbb{N}} \mathcal{F}_{n}^{\sigma}$ como o conjunto de toda as $\sigma$-flags finitas a menos de isomorfismo.

Denotamos 0 como o único tipo de tamanho 0 e observe que uma 0 -flag é essencialmente um torneio não rotulado. Notemos que para todo tipo $\sigma$ o conjunto $\mathcal{F}_{|\sigma|}^{\sigma}$ tem somente o elemento $\left(\sigma\right.$, id), onde id é a função identidade, que denotamos por $1_{\sigma}$.

Se $F=(T, \theta)$ é uma $\sigma$-flag e $W \subset V(T)$ é tal que $\operatorname{im}(\theta) \subset W$ (i.e., o conjunto $W$ contém todos os vértices rotulados), então definimos a subflag induzida por $W$ em $F$ como a flag $\left.F\right|_{W}=$ $\left(\left.T\right|_{W}, \theta\right)$, onde $\left.T\right|_{W}$ é o subtorneio induzido por $W$ em $T$.

Agora podemos estender a notação de densidade para flags e também uma densidade mais geral para várias flags conjuntamente. 
Definição 4.3.1. Seja $\sigma$ um tipo de tamanho $k$ e $\ell, \ell_{1}, \ell_{2}, \ldots, \ell_{t} \geq k$ inteiros tais que

$$
\left(\sum_{i=1}^{t} \ell_{i}\right)-(t-1) k \leq \ell
$$

Sejam também $F=(M, \theta), F_{1}, F_{2}, \ldots, F_{t} \in \mathcal{F}^{\sigma}$ com tamanhos $\ell, \ell_{1}, \ell_{2}, \ldots, \ell_{t}$ respectivamente. A densidade conjunta de $F_{1}, F_{2}, \ldots, F_{t}$ em $F$, denotada por $p\left(F_{1}, F_{2}, \ldots, F_{t} ; F\right)$, é definida através do seguinte experimento aleatório. Escolha uniformemente ao acaso os subconjuntos disjuntos $\boldsymbol{W}_{\mathbf{1}}, \boldsymbol{W}_{\mathbf{2}}, \ldots, \boldsymbol{W}_{\boldsymbol{t}}$ de $V(F) \backslash \operatorname{im}(\theta)$ sujeito a $\left|\boldsymbol{W}_{\boldsymbol{i}}\right|=\ell_{i}-k$ para todo $i \in[t]$ e defina

$$
p\left(F_{1}, F_{2}, \ldots, F_{t} ; F\right)=\mathbb{P}\left[\forall i \in[t],\left.F\right|_{\operatorname{im}(\theta) \cup \boldsymbol{W}_{\boldsymbol{i}}} \cong F_{i}\right]
$$

Também estendemos p linearmente para cada uma de suas coordenadas.

Agora podemos apresentar a álgebra de flags de um tipo $\sigma$.

Proposição 4.3.2 (Razborov [Raz07, Lemma 2.4]). Seja $\sigma$ um tipo de tamanho $k$ e denote $\mathcal{A}^{\sigma}=\mathbb{R} \mathcal{F}^{\sigma} / \mathcal{K}^{\sigma}$ como o quociente do conjunto $\mathbb{R} \mathcal{F}^{\sigma}$ de todas as combinações lineares formais dos elementos de $\mathcal{F}^{\sigma}$ pelo subespaço linear $\mathcal{K}^{\sigma}$ gerado pelos elementos da forma

$$
\widetilde{F}-\sum_{F \in \mathcal{F}_{\ell}^{\sigma}} p(\widetilde{F} ; F) F
$$

onde $\ell \geq|\widetilde{F}|$. Defina também o produto linear $\cdot: \mathcal{A}^{\sigma} \times \mathcal{A}^{\sigma} \rightarrow \mathcal{A}^{\sigma}$ através

$$
F_{1} \cdot F_{2}=\sum_{F \in \mathcal{F}_{\ell}^{\sigma}} p\left(F_{1}, F_{2} ; F\right) F
$$

onde $F_{1}, F_{2} \in \mathcal{F}^{\sigma}$ e $\ell \geq\left|F_{1}\right|+\left|F_{2}\right|-k$.

Sob essas condições, este produto é bem definido e o conjunto $\mathcal{A}^{\sigma}$ equipado com este produto (e com a adição usual) é uma álgebra comutativa associativa sobre $\mathbb{R}$ com unidade $1_{\sigma}$.

Denotamos $\operatorname{Hom}\left(\mathcal{A}^{\sigma}, \mathbb{R}\right)$ como o conjunto de todos homomorfismo da $\mathbb{R}$-álgebra de $\mathcal{A}^{\sigma}$ para $\mathbb{R}$ e definimos o conjunto dos homomorfismo positivos como

$$
\operatorname{Hom}^{+}\left(\mathcal{A}^{\sigma}, \mathbb{R}\right)=\left\{\phi \in \operatorname{Hom}\left(\mathcal{A}^{\sigma}, \mathbb{R}\right): \forall F \in \mathcal{A}^{\sigma}, \phi(F) \in[0,1]\right\}
$$

A seguir definimos a noção de sequência convergente de flags. 
Definição 4.3.3. Seja $\left(F_{n}\right)_{n \in \mathbb{N}}$ uma sequência de $\sigma$-flags. A sequência $\left(F_{n}\right)_{n \in \mathbb{N}}$ é dita crescente se $\left|F_{n}\right|<\left|F_{n+1}\right|$ para todo $n \in \mathbb{N}$. A sequência $\left(F_{n}\right)_{n \in \mathbb{N}}$ é dita convergente se é crescente e para toda $\sigma$-flag fixa $F \in \mathcal{F}^{\sigma}$, a sequência de números reais $\left(p\left(F ; F_{n}\right)\right)_{n \in \mathbb{N}}$ é convergente. Se $\phi \in \operatorname{Hom}^{+}\left(\mathcal{A}^{\sigma}, \mathbb{R}\right)$ é um homomorfismo, dizemos que a sequência $\left(F_{n}\right)_{n \in \mathbb{N}}$ converge para $\phi$ se é convergente e

$$
\lim _{n \rightarrow \infty} p\left(F ; F_{n}\right)=\phi(F)
$$

para toda $\sigma$-flag $F \in \mathcal{F}^{\sigma}$.

Observe que toda sequência crescente de flags tem uma subsequência convergente. O próximo teorema afirma que o conjunto dos homomorfismos positivos $\operatorname{Hom}^{+}\left(\mathcal{A}^{\sigma}, \mathbb{R}\right)$ captura precisamente os limites das sequências de $\sigma$-flags convergentes.

Teorema 4.3.4 (Lovász-Szegedy [LS06], Razborov [Raz07, Theorem 3.3]). Toda sequência convergente de $\sigma$-flags converge para um homomorfismo positivo em $\operatorname{Hom}^{+}\left(\mathcal{A}^{\sigma}, \mathbb{R}\right)$. Por outro lado, para todo homomorfismo positivo $\phi \in \operatorname{Hom}^{+}\left(\mathcal{A}^{\sigma}, \mathbb{R}\right)$, existe uma sequência de $\sigma$-flags que converge para $\phi$.

Lembramos que estamos interessados em maximizar a densidade assintótica de um torneio fixo $T$. Na linguagem de álgebra de flags, isso significa que estamos interessados no seguinte problema.

Problema 4.3.5. Dado um torneio fixo $T \in \mathcal{T}$, compute

$$
\max \left\{\phi(T): \phi \in \operatorname{Hom}^{+}\left(\mathcal{A}^{\sigma}, \mathbb{R}\right)\right\}
$$

Observação 4.3.6. Aqui usamos max em vez de sup porque se sabe que $\operatorname{Hom}^{+}\left(\mathcal{A}^{\sigma}, \mathbb{R}\right)$ é compacto.

\subsubsection{O método semidefinido}

Prover limitantes inferiores para o Problema 4.3.5 é fácil. De fato, toda sequência crescente de torneios $\left(T_{n}\right)_{n \in \mathbb{N}}$ nos dá um limitante inferior

$$
\limsup _{n \rightarrow \infty} p\left(T ; T_{n}\right)
$$


A parte difícil desse problema é computar limitantes superiores não triviais. Uma primeira e ingênua forma de fazer isto é descrita a seguir. Se $\phi \in \operatorname{Hom}^{+}\left(\mathcal{A}^{\sigma}, \mathbb{R}\right)$, então a Proposição 4.3 .2 nos diz que

$$
\begin{aligned}
\phi(T) & =\sum_{T^{\prime} \in \mathcal{T}_{\ell}} p\left(T ; T^{\prime}\right) \phi\left(T^{\prime}\right) \\
& \leq\left(\max _{T^{\prime} \in \mathcal{T}_{\ell}} p\left(T ; T^{\prime}\right)\right) \sum_{T^{\prime} \in \mathcal{T}_{\ell}} \phi\left(T^{\prime}\right)=\left(\max _{T^{\prime} \in \mathcal{T}_{\ell}} p\left(T ; T^{\prime}\right)\right) \phi\left(1_{0}\right)=\left(\max _{T^{\prime} \in \mathcal{T}_{\ell}} p\left(T ; T^{\prime}\right)\right),
\end{aligned}
$$

para todo $\ell \geq|T|$, pois $\phi$ é linear e $1_{0}=\sum_{T^{\prime} \in \mathcal{T}_{\ell}} T^{\prime}$.

Entretanto, em geral esse limitante é muito fraco para encontrar valores extremais. A seguir, apresentaremos o método semidefinido. Este método é construído a partir do argumento apresentado anteriormente 4.2, mas podemos obter limitantes muito melhores para o Problema 4.3.5.

Começaremos definindo algumas notações da álgebra de flags.

Definição 4.3.7. Seja $\sigma$ um tipo. Definimos o cone semântico do tipo $\sigma$ como o conjunto

$$
\mathcal{C}_{\mathrm{sem}}\left(\mathcal{F}^{\sigma}\right)=\left\{f \in \mathcal{A}^{\sigma}: \forall \phi \in \operatorname{Hom}^{+}\left(\mathcal{A}^{\sigma}, \mathbb{R}\right), \phi(f) \geq 0\right\}
$$

Ou seja, o cone semântico é conjunto dos elementos "positivos" de $\mathcal{A}^{\sigma}$ com respeito aos homomorfismos positivos. Também definimos o cone ordinário do tipo $\sigma$ como o conjunto

$$
\mathcal{C}\left(\mathcal{F}^{\sigma}\right)=\left\{\sum_{i=1}^{t} F_{i} \cdot f_{i}^{2}: t \in \mathbb{N} \text { e } F_{1}, F_{2}, \ldots, F_{t} \in \mathcal{F}^{\sigma} \text { e } f_{1}, f_{2}, \ldots, f_{t} \in \mathcal{A}^{\sigma}\right\}
$$

Finalmente, definimos a relação de pré-ordem $\leq_{\sigma}$ sobre $\mathcal{A}^{\sigma}$ como a seguir

$$
f \leq_{\sigma} g \Longleftrightarrow g-f \in \mathcal{C}_{\mathrm{sem}}\left(\mathcal{F}^{\sigma}\right)
$$

Trivialmente temos $\mathcal{F}^{\sigma} \subset \mathcal{C}\left(\mathcal{F}^{\sigma}\right) \subset \mathcal{C}_{\text {sem }}\left(\mathcal{F}^{\sigma}\right)$.

A ideia do método semidefinido é usar elementos de $\mathcal{C}_{\text {sem }}\left(\mathcal{F}^{0}\right)$ para compensar os valores grandes de $p\left(T ; T^{\prime}\right)$ em (4.2) como a seguir. Se $g \in \mathcal{C}_{\text {sem }}\left(\mathcal{F}^{0}\right)$, então

$$
T \leq_{0} T+g=\sum_{T^{\prime} \in \mathcal{T}_{\ell}}\left(p\left(T ; T^{\prime}\right)+p\left(g ; T^{\prime}\right)\right) T^{\prime}
$$




$$
\leq_{0}\left(\max _{T^{\prime} \in \mathcal{T}_{\ell}}\left(p\left(T ; T^{\prime}\right)+p\left(g ; T^{\prime}\right)\right)\right) \sum_{T^{\prime} \in \mathcal{T}_{\ell}} T^{\prime}=\left(\max _{T^{\prime} \in \mathcal{T}_{\ell}}\left(p\left(T ; T^{\prime}\right)+p\left(g ; T^{\prime}\right)\right)\right) 1_{0}
$$

onde $\ell \in \mathbb{N}$ é suficientemente grande (de modo que podemos escrever $g$ como uma combinação de torneios de tamanho menor que $\ell$ ). Procuramos escolher $g$ de modo que $p\left(g ; T^{\prime}\right)$ tenha valor negativo quando o valor $p\left(T ; T^{\prime}\right)$ for grande e $p\left(g ; T^{\prime}\right)$ suficientemente positivo para garantir $g \in$ $\mathcal{C}_{\text {sem }}\left(\mathcal{F}^{0}\right)$ quando $p\left(T ; T^{\prime}\right)$ é pequeno.

Entretanto, decidir se um $g$ arbitrário é um elemento de $\mathcal{C}_{\text {sem }}\left(\mathcal{F}^{\sigma}\right)$ é difícil (de fato, nosso problema é exatamente provar que $c 1_{0}-T \in \mathcal{C}_{\text {sem }}\left(\mathcal{F}^{0}\right)$ para um certo $\left.c\right)$. Agora vamos definir o operador descendente, que nos ajudará a obter elementos de $\mathcal{C}_{\text {sem }}\left(\mathcal{F}^{0}\right)$ a partir de elementos de $\mathcal{C}_{\text {sem }}\left(\mathcal{F}^{\sigma}\right)$.

Definição 4.3.8. Seja $\sigma$ um tipo de tamanho $k$ e $F=(T, \theta)$ uma $\sigma$-flag. Denotamos o torneio subjacente de $F$ por $\left.F\right|_{0}=T$ e definimos o fator de normalização de $F\left(\operatorname{denotado} q_{\sigma}(F)\right.$ ) através do seguinte experimento aleatório: escolhemos uniformemente ao acaso uma função injetiva $\boldsymbol{\theta}:[k] \rightarrow V\left(\left.F\right|_{0}\right)$ e definimos

$$
q_{\sigma}(F)=\mathbb{P}\left[\left(\left.F\right|_{0}, \boldsymbol{\theta}\right) \cong F\right]
$$

Definimos o operador descendente $\llbracket \cdot \rrbracket_{\sigma}$ como a seguir

$$
\llbracket F \rrbracket_{\sigma}=\left.q_{\sigma}(F) F\right|_{0} \quad \in \mathcal{A}^{0}
$$

e o estendemos linearmente para combinações de $\sigma$-flags.

Teorema 4.3.9 (Razborov [Raz07, Teoremas 2.5 e 3.1a]). O operador descendente $\llbracket \cdot \rrbracket_{\sigma}$ é bem definido como um operador $\mathcal{A}^{\sigma} \rightarrow \mathcal{A}^{0}$ e temos

$$
\llbracket \mathcal{C}_{\mathrm{sem}}\left(\mathcal{F}^{\sigma}\right) \rrbracket_{\sigma} \subset \mathcal{C}_{\mathrm{sem}}\left(\mathcal{F}^{0}\right)
$$

Esse teorema nos permite escolher $g$ de (4.3) no conjunto "mais fácil" $\llbracket \mathcal{C}\left(\mathcal{F}^{\sigma}\right) \rrbracket_{\sigma}$ para algum tipo $\sigma$. Isso reduz o problema ao de encontrar uma matriz positiva semidefinida da forma descrita a seguir. Fixe um tipo $\sigma$ de tamanho $k$, uma $\sigma$-flag $F^{\prime}$ e sejam $\tilde{\ell}$ e $\ell$ inteiros tais que $k \leq \widetilde{\ell}$ e $\left|F^{\prime}\right|+2 \tilde{\ell}-2 k \leq \ell$. 
Para todo $v \in \mathbb{R}^{\mathcal{F}} \tilde{\bar{\ell}}$ indexado por $\mathcal{F}_{\tilde{\ell}}^{\sigma}$, definimos

$$
F(v)=\sum_{F \in \mathcal{F}_{\bar{\ell}}^{\sigma}} v_{F} F \in \mathcal{A}^{\sigma}
$$

Analogamente, para toda matriz $Q$ indexada por $\mathcal{F}_{\widetilde{\ell}}^{\sigma} \times \mathcal{F}_{\widetilde{\ell}}^{\sigma}$, definimos

$$
F(Q)=\sum_{F_{1}, F_{2} \in \mathcal{F}_{\bar{\ell}}^{\sigma}} Q_{F_{1} F_{2}} F_{1} F_{2} \in \mathcal{A}^{\sigma}
$$

Observe que, se $Q$ é positiva semidefinida $(Q \succeq 0)$, então pelo Teorema Espectral existem vetores $v_{1}, v_{2}, \ldots, v_{r} \in \mathbb{R}^{\mathcal{F}_{\bar{\ell}}^{\sigma}}$ tais que

$$
Q=\sum_{i=1}^{r} v_{i} v_{i}^{\top}
$$

o que significa que

$$
F(Q)=\sum_{i=1}^{r} F\left(v_{i}\right)^{2}
$$

Assim, como $F^{\prime} \cdot F(Q) \in \mathcal{C}\left(\mathcal{F}^{\sigma}\right)$, temos que $\llbracket F^{\prime} \cdot F(Q) \rrbracket_{\sigma} \in \mathcal{C}_{\text {sem }}\left(\mathcal{F}^{0}\right)$. Portanto, fazendo $g=$ $\llbracket F^{\prime} \cdot F(Q) \rrbracket_{\sigma}$ em $(4.3)$ e se considerarmos todas as possíveis matrizes $Q$, obtemos o seguinte programa semidefinido.

$\min y$

$$
\begin{array}{ll}
\text { s.t. } & p\left(T, T^{\prime}\right)+\sum_{F \in \mathcal{F}_{\ell}^{\sigma}} \sum_{F_{1}, F_{2} \in \mathcal{F}_{\tilde{\ell}}^{\sigma}} Q_{F_{1} F_{2}} p\left(F^{\prime}, F_{i}, F_{j} ; F\right) p\left(\llbracket F \rrbracket_{\sigma} ; T^{\prime}\right) \leq y \quad \forall T^{\prime} \in \mathcal{T}_{\ell} ; \\
& Q \in \mathbb{R}^{\mathcal{F}_{\tilde{\ell}}^{\sigma} \times \mathcal{F}_{\tilde{\ell}}^{\sigma}} \text { é positiva semidefinida; }
\end{array}
$$

cujas soluções têm valores que são limitantes superiores para o valor no Problema 4.3.5.

De fato, podemos ainda tomar $g=\sum_{i=1}^{m} g_{i}$ em (4.3), onde $g_{i}=\llbracket F_{i}^{\prime} \cdot F\left(Q_{i}\right) \rrbracket_{\sigma_{i}}$ para algum tipo fixo $\sigma_{i}$, alguma $\sigma_{i}$-flag $F_{i}^{\prime}$ e alguma matriz positiva semidefinida $Q_{i}$ indexada por $\mathcal{F}_{\ell_{i}}^{\sigma_{i}} \times \mathcal{F}_{\ell_{i}}^{\sigma_{i}}$. Apresentamos o programa semidefinido resultante na Proposição 4.3 .10 a seguir.

Proposição 4.3.10 ([Raz10]). Seja $T \in \mathcal{T}$ um torneio, e sejam $\sigma_{1}, \sigma_{2}, \ldots, \sigma_{m}$ tipos de tamanhos $k_{1}, k_{2}, \ldots, k_{m}$ respectivamente e para cada $t \in[m]$ seja $F_{t}^{\prime} \in \mathcal{F}^{\sigma_{t}}$ uma $\sigma_{t}$-flag. Sejam 
também $\ell_{1}, \ell_{2}, \ldots, \ell_{m}, \ell$ inteiros tais que

$$
k_{t} \leq \ell_{t} ; \quad\left|F_{t}^{\prime}\right|+2 \ell_{t}-2 k_{t} \leq \ell
$$

para todo $t \in[m]$ e tal que $|T| \leq \ell$.

Sob estas circunstâncias, todo valor de toda solução do programa semidefinido

$\min y$

$$
\begin{array}{ll}
\text { s.t. } & p\left(T, T^{\prime}\right)+\sum_{t=1}^{m} \sum_{F \in \mathcal{F}_{\ell}^{\sigma_{t}}} \sum_{F_{1}, F_{2} \in \mathcal{F}_{\ell_{t}}^{\sigma_{t}}} Q_{F_{1} F_{2}}^{(t)} p\left(F_{t}^{\prime}, F_{i}, F_{j} ; F\right) p\left(\llbracket F \rrbracket_{\sigma_{t}} ; T^{\prime}\right) \leq y \quad \forall T^{\prime} \in \mathcal{T}_{\ell} ; \\
& Q^{(t)} \in \mathbb{R}^{\mathcal{F}_{\ell_{t}}^{\sigma_{t}} \times \mathcal{F}_{\ell_{t}}^{\sigma_{t}}} \text { é positiva semidefinida } \quad \forall t \in[m] ;
\end{array}
$$

é um limitante superior para o valor no Problema 4.3.5, ou seja, se $V$ é o valor da solução de (4.4), então

$$
\max \left\{\phi(T): \phi \in \operatorname{Hom}^{+}\left(\mathcal{A}^{0}, \mathbb{R}\right)\right\} \leq V .
$$

Neste texto, todas as instâncias de (4.4) estarão com $F_{t}^{\prime}=1_{\sigma_{t}}$ para todo $t \in[m]$. Ademais, quando usamos a Proposição 4.3.10 para obter limitantes superiores para o Problema 4.3.5, denotaremos cada uma das $Q^{(t)}$ em (4.4) por $Q\left(T, \sigma_{t}\right)$, para todo $t \in[m]$, como um lembrete de que problema estamos resolvendo e de qual o tipo envolvido. Além disso, para cada $T^{\prime} \in \mathcal{T}_{\ell}$, definimos

$$
c\left(Q\left(T, \sigma_{t}\right) ; T^{\prime}\right)=p\left(\llbracket F\left(Q\left(T, \sigma_{t}\right)\right) \rrbracket_{\sigma_{t}} ; T^{\prime}\right)=\sum_{F \in \mathcal{F}_{\ell}^{\sigma_{t}}} \sum_{F_{1}, F_{2} \in \mathcal{F}_{\ell_{t}}^{\sigma_{t}}} Q\left(T, \sigma_{t}\right)_{F_{1} F_{2}} p\left(F_{i}, F_{j} ; F\right) p\left(\llbracket F \rrbracket_{\sigma_{t}} ; T^{\prime}\right)
$$

e seja

$$
c\left(T ; T^{\prime}\right)=\sum_{t=1}^{m} c\left(Q\left(T, \sigma_{t}\right) ; T^{\prime}\right),
$$

de modo que (4.4) torna-se

$\min y$ 


$$
\begin{aligned}
& \text { s.t. } p\left(T, T^{\prime}\right)+c\left(T ; T^{\prime}\right) \leq y \quad \forall T^{\prime} \in \mathcal{T}_{\ell} \text {; } \\
& c\left(T ; T^{\prime}\right)=\sum_{t=1}^{m} c\left(Q\left(T, \sigma_{t}\right) ; T^{\prime}\right) \quad \forall T^{\prime} \in \mathcal{T}_{\ell} ; \\
& c\left(Q\left(T, \sigma_{t}\right) ; T^{\prime}\right)=\sum_{F \in \mathcal{F}_{\ell}^{\sigma_{t}}} \sum_{F_{1}, F_{2} \in \mathcal{F}_{\ell_{t}}^{\sigma_{t}}} Q\left(T, \sigma_{t}\right)_{F_{1} F_{2}} p\left(F_{i}, F_{j} ; F\right) p\left(\llbracket F \rrbracket_{\sigma_{t}} ; T^{\prime}\right) ;
\end{aligned}
$$

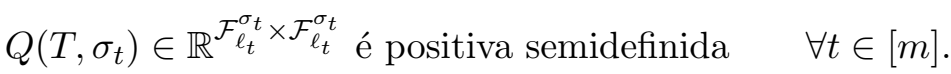

\subsubsection{Extraindo mais informações do método semidefinido}

Nesta seção, revisaremos algumas técnicas em álgebra de flags para obter informações sobre os homomorfismos extremais do Problema 4.3.5 através de uma solução justa do programa semidefinido (4.4) (veja também a versão mais geral (4.5)). Novamente, trabalharemos somente com a teoria dos torneios, mas as técnicas que apresentaremos podem ser utilizadas em contextos mais gerais.

A primeira técnica é usada para provar que o torneio $T^{\prime}$ correspondente às restrições que não valem na igualdade em (4.4) deve ter densidade zero no homomorfismo extremal.

Proposição 4.3.11. Sejam $T \in \mathcal{T}$ um torneio e

$$
c=\max \left\{\phi(T): \phi \in \operatorname{Hom}^{+}\left(\mathcal{A}^{0}, \mathbb{R}\right)\right\} .
$$

Se $\ell \geq|T|$ e $g \in \mathcal{C}_{\text {sem }}\left(\mathcal{F}^{0}\right)$ são tais que

$$
\max \left\{p\left(T+g ; T^{\prime}\right): T^{\prime} \in \mathcal{T}_{\ell}\right\}=c,
$$

e $\phi \in \operatorname{Hom}^{+}\left(\mathcal{A}^{0}, \mathbb{R}\right)$ é extremal para $T$ (isto é, se $\phi(T)=c$ ), então

$$
\phi\left(T^{\prime}\right)=0,
$$

para todo $T^{\prime} \in \mathcal{T}_{\ell}$ tal que $p\left(T+g ; T^{\prime}\right)<c$.

Demonstração. Pelo método semidefinido da Seção 4.3.2, sabemos que

$$
c=\phi(T) \leq \phi(T+g)=\sum_{T^{\prime} \in \mathcal{T}_{\ell}} p\left(T+g ; T^{\prime}\right) \phi\left(T^{\prime}\right)
$$




$$
\leq\left(\max _{T^{\prime} \in \mathcal{T}_{\ell}} p\left(T+g ; T^{\prime}\right)\right) \sum_{T^{\prime} \in \mathcal{T}_{\ell}} \phi\left(T^{\prime}\right)=c \phi\left(1_{0}\right)=c .
$$

Consequentemente, devemos ter igualdade em todas inequações. Em particular, igualdade no lugar da última desigualdade implica que

$$
\sum_{T^{\prime} \in \mathcal{T}_{\ell}} p\left(T+g ; T^{\prime}\right) \phi\left(T^{\prime}\right)=c \sum_{T^{\prime} \in \mathcal{T}_{\ell}} \phi\left(T^{\prime}\right)
$$

e como $\phi\left(T^{\prime}\right) \geq 0$ para $T^{\prime} \in \mathcal{T}_{\ell}$, temos

$$
\phi\left(T^{\prime}\right)\left(c-p\left(T+g ; T^{\prime}\right)\right)=0,
$$

para todo $T^{\prime} \in \mathcal{T}_{\ell}$. Assim, o resultado segue.

Para a próxima técnica, precisamos da noção de extensão de um homomorfismo, então apresentaremos a seguir o principal teorema sobre o assunto.

Teorema 4.3.12 (Razborov [Raz07, Teorema 3.5]). Se $\sigma$ é um tipo e $\phi \in \operatorname{Hom}^{+}\left(\mathcal{A}^{0}, \mathbb{R}\right)$ é

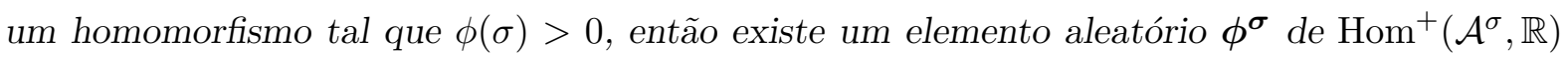
(chamado homomorfismo aleatório) tal que

$$
\mathbb{E}\left[\phi^{\sigma}(f)\right]=\frac{\phi\left(\llbracket f \rrbracket_{\sigma}\right)}{\phi\left(\llbracket 1_{\sigma} \rrbracket_{\sigma}\right)},
$$

para todo $f \in \mathcal{A}^{\sigma}$.

A próxima técnica afirma que, se o elemento $\llbracket F \cdot f^{2} \rrbracket_{\sigma}$ foi usado em uma solução exata de (4.4), então devemos ter $\phi^{\sigma}(F \cdot f)=0$ quase certamente para todo homomorfismo extremal $\phi \in$ $\operatorname{Hom}^{+}\left(\mathcal{A}^{0}, \mathbb{R}\right)$.

Proposição 4.3.13. Com as definições e notações da Proposição 4.3.10, seja

$$
c=\max \left\{\phi(T): T \in \operatorname{Hom}^{+}\left(\mathcal{A}^{0}, \mathbb{R}\right)\right\},
$$

suponha que a solução ótima $\left(Q^{(t)}\right)_{t=1}^{m}$ de (4.4) tem valor $c$ e escrevemos

$$
Q^{(t)}=\sum_{i=1}^{r_{t}} v_{i}^{(t)}\left(v_{i}^{(t)}\right)^{\top}
$$


para todo $t \in[m]$.

Sob essas circunstâncias, se $\phi \in \operatorname{Hom}^{+}\left(\mathcal{A}^{0}, \mathbb{R}\right)$ é extremal para $T$, isto é, se $\phi(T)=c$, então para todo $t \in[m] \operatorname{com} \phi\left(\sigma_{t}\right)>0$ e todo $i \in\left[r_{t}\right]$, temos

$$
\phi^{\sigma}\left(F_{t}^{\prime} \cdot F\left(v_{i}^{(t)}\right)\right)=0
$$

quase certamente.

Demonstração. Recorde o método semidefinido da Seção 4.3.2. Sabemos que

$$
\begin{aligned}
c=\phi(T) & \leq \phi(T)+\sum_{i=1}^{m} \phi\left(\llbracket F_{t}^{\prime} \cdot F\left(Q^{(t)}\right) \rrbracket_{\sigma_{t}}\right) \\
& =\sum_{T^{\prime} \in \mathcal{T}_{\ell}}\left(p\left(T ; T^{\prime}\right)+\sum_{i=1}^{m} p\left(\llbracket F_{t}^{\prime} \cdot F\left(Q^{(t)}\right) \rrbracket_{\sigma_{t}} ; T^{\prime}\right)\right) \phi\left(T^{\prime}\right) \\
& \leq \max _{T^{\prime} \in \mathcal{T}_{\ell}}\left(p\left(T ; T^{\prime}\right)+\sum_{i=1}^{m} p\left(\llbracket F_{t}^{\prime} \cdot F\left(Q^{(t)}\right) \rrbracket_{\sigma_{t}} ; T^{\prime}\right)\right) \phi\left(1_{0}\right) \\
& =c .
\end{aligned}
$$

Consequentemente, devemos ter igualdade em todas inequações. Em particular, igualdade na primeira inequação implica que

$$
\sum_{i=1}^{m} \phi\left(\llbracket F_{t}^{\prime} \cdot F\left(Q^{(t)}\right) \rrbracket_{\sigma_{t}}\right)=0,
$$

e como $\llbracket F_{t}^{\prime} \cdot F\left(Q^{(t)}\right) \rrbracket_{\sigma_{t}} \in \mathcal{C}_{\text {sem }}\left(\mathcal{F}^{0}\right)$ para todo $t \in[m]$, temos que

$$
\phi\left(\llbracket F_{t}^{\prime} \cdot F\left(Q^{(t)}\right) \rrbracket_{\sigma_{t}}\right)=0
$$

para todo $t \in[m]$.

Agora fixe $t \in[m]$ tal que $\phi\left(\sigma_{t}\right)>0$ e recorde que

$$
\llbracket F_{t}^{\prime} \cdot F\left(Q^{(t)}\right) \rrbracket_{\sigma_{t}}=\sum_{i=1}^{r_{t}} \llbracket F_{t}^{\prime} \cdot F\left(v_{i}^{(t)}\right)^{2} \rrbracket_{\sigma_{t}}
$$


Isso juntamente com (4.6) implica que

$$
\phi\left(\llbracket F_{t}^{\prime} \cdot F\left(v_{i}^{(t)}\right)^{2} \rrbracket_{\sigma_{t}}\right)=0
$$

Do Teorema 4.3.12, temos

$$
\mathbb{E}\left[\phi^{\sigma_{t}}\left(F_{t}^{\prime} \cdot F\left(v_{i}^{(t)}\right)^{2}\right)\right]=0,
$$

e como esta variável é (quase certamente) não negativa, temos

$$
\phi^{\sigma_{t}}\left(F_{t}^{\prime} \cdot F\left(v_{i}^{(t)}\right)\right)=0
$$

quase certamente, como desejado.

\subsubsection{Torneios, tipos e flags usados}

Ao longo deste texto, denotamos o torneio transitivo de tamanho $k$ por $\operatorname{Tr}_{k}$, onde dizemos que um torneio $T$ é transitivo se, para todo par de arco $(x, y) \in A(T)$ e $(y, z) \in A(T)$, temos que $(x, z) \in A(T)$. Também denotamos (veja Figura 4.2):

- O circuito dirigido com 3 vértices por $\vec{C}_{3}$;

- O único torneio de tamanho 4 que tem um circuito com 4 vértices por $R_{4}$;

- O único torneio de tamanho 4 com sequência de grau de saída $(1,1,1,3)$ por $W_{4}$;

- O único torneio de tamanho 4 com sequência de grau de saída $(0,2,2,2)$ por $L_{4}$.

Também usaremos a notação da Figura 4.3 para os torneios não isomorfos de tamanho 5 . Ademais, definimos os seguintes tipos (veja Figura 4.2).

- O único tipo de tamanho 1 é denotado por 1 ;

- O tipo com tamanho 2 onde o arco é orientado do vértice com rótulo 1 para o vértice com rótulo 2 é denotado por $A$;

- O tipo com tamanho 3 isomorfo ao $\operatorname{Tr}_{3}$ tal que o "vencedor" tem rótulo 1 e o "perdedor" tem rótulo 3 é denotado por $\operatorname{Tr}_{3}^{*}$; 


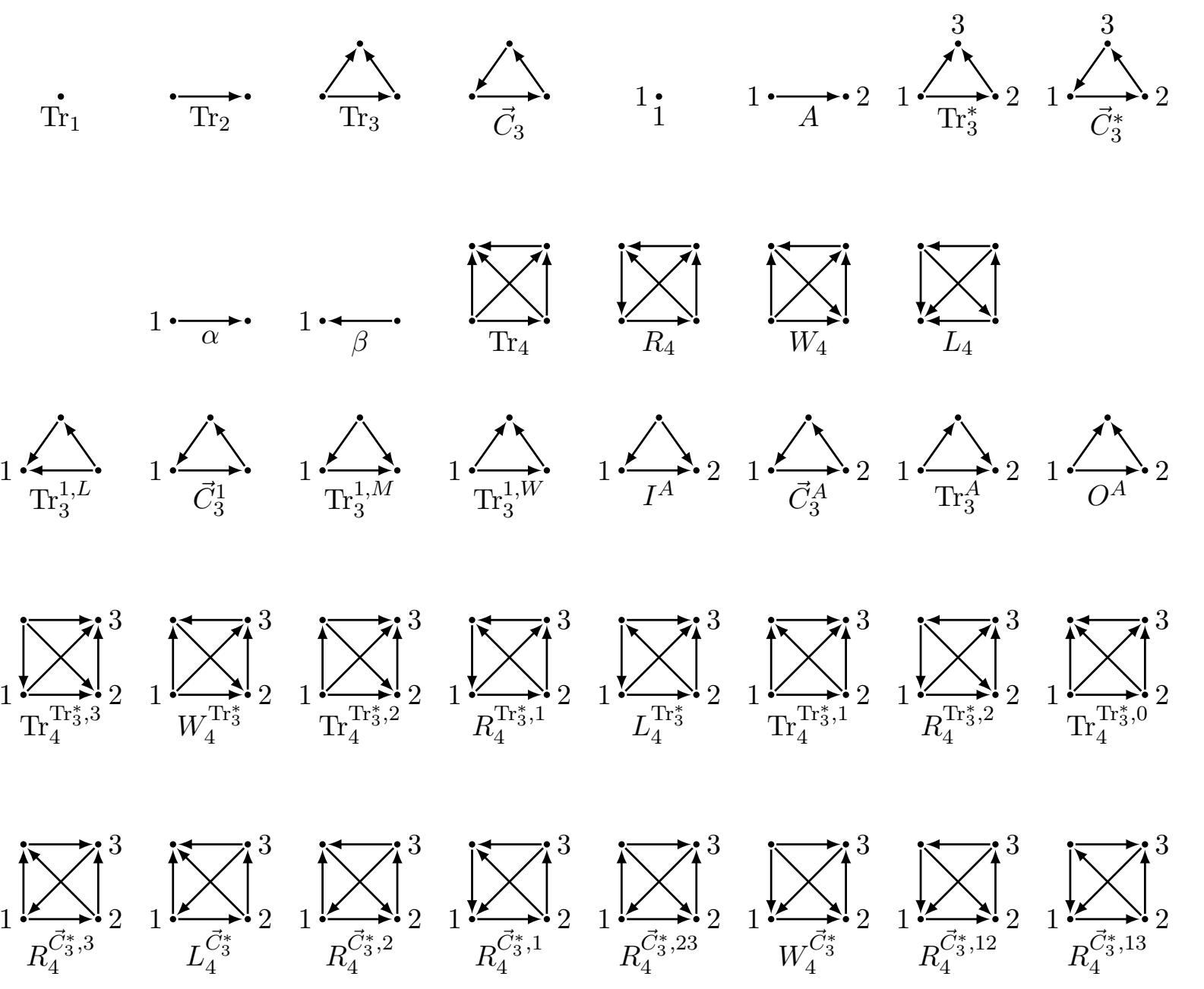

Figura 4.2: Tipos e flags usados com tamanhos no máximo 4.

- O tipo com tamanho 3 isomorfo ao $\vec{C}_{3}$ tal que o vértice com rótulo 1 chega no vértice com rótulo 2 é denotado por $\vec{C}_{3}^{*}$

Se $T$ é um torneio e $\sigma$ é um tipo tal que existe exatamente uma $\sigma$-flag $F$ tal que $\left.F\right|_{0}=T$, então denotaremos tal flag por $T^{\sigma}$. Observe que isso define unicamente as seguintes flags.

$$
\vec{C}_{3}^{1}, \vec{C}_{3}^{A}, W_{4}^{\operatorname{Tr}_{3}^{*}}, L_{4}^{\operatorname{Tr}_{3}^{*}}, W_{4}^{\vec{C}_{3}^{*}}, L_{4}^{\vec{C}_{3}^{*}}
$$

Para as flags restantes usaremos a notação da Figura 4.2. Vamos comentar somente o raciocínio por de trás da nossa notação.

- A notação para as flags $O^{A}$ e $I^{A}$ são destinadas a serem um mnemônico para vizinhança comum de saída (out-neighbourhood em inglês) e vizinhança comum de entrada (inneighbourhood em inglês) respectivamente; 


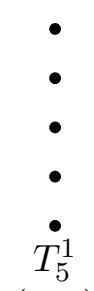

$\left(\operatorname{Tr}_{5}\right)$
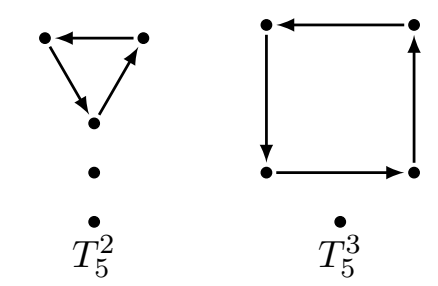

$\dot{T}_{5}^{3}$
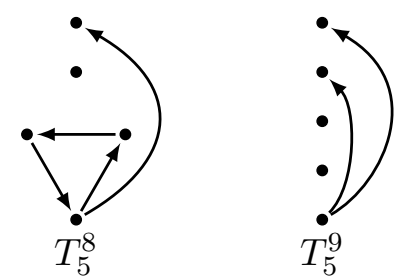
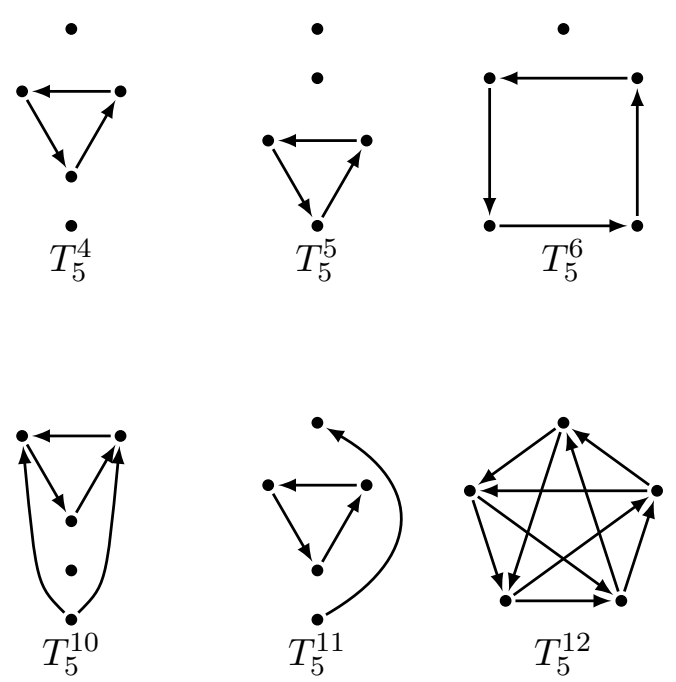

$\left(R_{5}\right)$

Figura 4.3: Torneios de tamanho 5. Os arcos omitidos são todos orientados para baixo.

- A flag $\operatorname{Tr}_{3}^{A}$ não é a única $A$-flag sobre $\operatorname{Tr}_{3}$, mas apesar disto usamos esta notação porque $\operatorname{Tr}_{3}^{A}$ é a única $A$-flag sobre $\operatorname{Tr}_{3}$ restante;

- As $\operatorname{Tr}_{3}^{*}$-flags sobre $\operatorname{Tr}_{4}$ e $R_{4}$ são unicamente determinadas pelo grau de saída $d$ dos vértices não rotulados e como tal, as denotamos nesse sentido por $\operatorname{Tr}_{4}^{\operatorname{Tr}_{3}^{*}, d}$ e $R_{4}^{\operatorname{Tr}_{3}^{*}, d}$;

- As $\vec{C}_{3}^{*}$-flags sobre $R_{4}$ são unicamente determinadas pela vizinhança de saída dos vértices não rotulados e como tal, as denotamos conforme a lista dos vértices na vizinhança de saída dos vértices não rotulados no sobrescrito.

\subsection{Limitantes inferiores}

Nesta seção, provamos os limitantes inferiores para os Teoremas 4.2.2, 4.2.1 e 4.2.3. Começamos por relembrar a definição de densidade rotulada em torneios.

Definição 4.4.1. Se $T_{1}$ e $T_{2}$ são torneios com $\left|T_{1}\right| \leq\left|T_{2}\right|$, então a densidade rotulada de $T_{1}$ em $T_{2}$ (denotada por $t_{\text {ind }}\left(T_{1} ; T_{2}\right)$ ) é a probabilidade de um mapeamento injetivo de $V\left(T_{1}\right)$ para $V\left(T_{2}\right)$ escolhido uniformemente ao acaso ser uma imersão de $T_{1}$ em $T_{2}$.

É fácil de ver que

$$
t_{\text {ind }}\left(T_{1} ; T_{2}\right)=\frac{\left|\operatorname{Aut}\left(T_{1}\right)\right|}{\left|T_{1}\right| !} p\left(T_{1} ; T_{2}\right)
$$


onde $\operatorname{Aut}\left(T_{1}\right)$ é o grupo de automorfismos de $T_{1}$.

Lema 4.4.2. Temos

$$
\lim _{n \rightarrow \infty} p\left(T_{5}^{7} ; R_{2 n+1}\right)=\frac{5}{16} ; \quad \quad \lim _{n \rightarrow \infty} p\left(T_{5}^{12} ; R_{2 n+1}\right)=\frac{1}{16} .
$$

Demonstração. Provaremos somente a afirmação para $T_{5}^{12}$, uma vez que a prova para $T_{5}^{7}$ é muito similar. Fixe $n \geq 2$ e seja $f: V\left(R_{5}\right) \rightarrow V\left(R_{2 n+1}\right)$ uma imersão de $R_{5}$ em $R_{2 n+1}$.

Suponha que o vértices 0 de $R_{5}$ é mapeado para o vértice 0 de $R_{n}$. Se o vértice 1 é mapeado para um vértice $i$, então $1 \leq i \leq n$ e o vértice 2 é mapeado para um vértice $j$ tal que $i+1 \leq j \leq n$. $\mathrm{O}$ vértice 3 tem de ser mapeado para um vértice $k$ tal que $n+1 \leq k \leq i+n$ (devido a $(3,0)$ e $(1,3)$ serem $\operatorname{arcos}$ de $\left.R_{5}\right)$. Finalmente, o vértice 4 tem que ser mapeado para um vértice $\ell$ tal que $i+n \leq \ell \leq j+n$ (devido a $(4,1)$ e $(2,4)$ serem arcos de $\left.R_{5}\right)$. Veja a Figura 4.4.

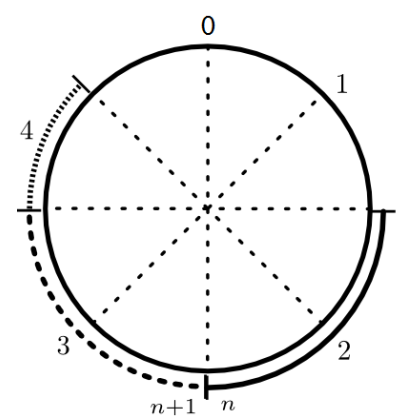

Figura 4.4: Possibilidade de imersão de $T_{5}^{12}=R_{5}$ em $R_{2 n+1}$.

Observe que nós podemos fixar as imagens dos vértices 0,1 e 2. O número de escolhas para o vértice 3 vem de $i$ e para o vértice 4 vem de $j-i+1$. Pela simetria de $R_{2 n+1}$, sabemos que isso é também o caso para qualquer outra escolha da imagem para o vértice 0 de $R_{5}$. Assim, temos

$$
\begin{aligned}
t_{\text {ind }}\left(R_{5} ; R_{2 n+1}\right) & =\frac{1}{(2 n+1)_{5}} \cdot(2 n+1) \sum_{i=1}^{n} \sum_{j=i+1}^{n} i(j-i+1) \\
& =\frac{1}{(2 n)_{4}} \sum_{i=1}^{n} \frac{i^{3}-(2 n-2) i^{2}+n(n-2) i}{2} \\
& =\frac{1}{(2 n)_{4}}\left(\frac{n^{4}}{24}+O\left(n^{3}\right)\right) \\
& =\frac{1}{3 \cdot 2^{7}}+O\left(\frac{1}{n}\right),
\end{aligned}
$$


onde $(\ell)_{k}=\ell(\ell-1) \cdots(\ell-k+1)$ denota o fatorial decrescente.

Portanto

$$
\lim _{n \rightarrow \infty} p\left(T_{5}^{12} ; R_{2 n+1}\right)=\lim _{n \rightarrow \infty} \frac{5 !}{5} t_{\text {ind }}\left(R_{5} ; R_{n}\right)=\frac{1}{16} .
$$

Agora vamos provar o limitante inferior para o Teorema 4.2.3.

Lema 4.4.3. Temos

$$
\lim _{n \rightarrow \infty} p\left(T_{5}^{9} ; \vec{C}_{3^{n}}^{3}\right)=\frac{3}{8} ; \quad \quad \lim _{n \rightarrow \infty} p\left(T_{5}^{11} ; \vec{C}_{3^{n}}^{3}\right)=\frac{1}{16} .
$$

Demonstração. Novamente provaremos somente a afirmação para $T_{5}^{9}$, devido a prova para $T_{5}^{11}$ ser bastante similar. Seja $T$ o torneio na Figura 4.5 que é isomorfo ao $T_{5}^{9}$. Relembre a definição

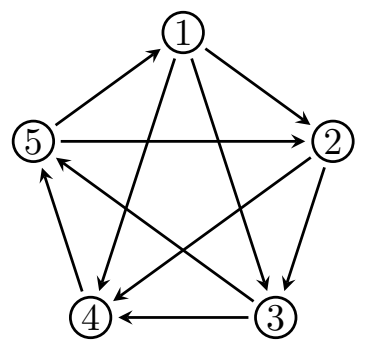

Figura 4.5: Torneio isomorfo a $T_{5}^{9}$.

de $\vec{C}_{3^{n}}^{3}$ na Seção 2.3 e sejam $A_{0}=\left[3^{n-1}\right], A_{1}=\left\{3^{n-1}+1,3^{n-1}+2, \ldots, 2 \cdot 3^{n-1}\right\}$ e $A_{2}=$ $\left\{2 \cdot 3^{n-1}+1,2 \cdot 3^{n-1}+2, \ldots, 3^{n}\right\}$.

Seja $F(n)$ o número de imersões de $T$ em $\vec{C}_{3^{n}}^{3}$. Cada uma das imersões ou mapeia todos os vértices de $T$ a um único $A_{i}$ ou mapeia 1 e 5 para alguma parte $A_{i}, 3$ e 4 para um $A_{(i+1) \bmod } 3$ e 2 para $A_{(i+2) \bmod 3}$. Desta forma, temos $F(1)=0$ e, para todo $n \geq 2$, temos

$$
F(n)=3\left(\begin{array}{c}
3^{n-1} \\
2
\end{array}\right)^{2} 3^{n-1}+3 F(n-1) \leq \frac{3^{5 n-4}}{4}+3 F(n-1) .
$$

Portanto, segue que

$$
F(n) \leq \sum_{i=0}^{n-1} 3^{i} \frac{3^{5(n-i)-4}}{4}=\frac{3^{5 n-4}}{4} \cdot \frac{1-3^{-4 n}}{1-3^{-4}}=\frac{3^{5 n}}{320}+O\left(3^{4 n}\right)
$$


Por outro lado, temos

$$
F(n) \geq \frac{3^{5 n-4}-3^{4 n-2}}{4}+3 F(n-1)
$$

consequentemente

$$
F(n) \geq \frac{3^{5 n}}{320}-\sum_{i=0}^{n-1} 3^{i} \frac{3^{2(n-i)-1}}{4}-O\left(3^{4 n}\right)=\frac{3^{5 n}}{320}-O\left(3^{4 n}\right)
$$

Portanto

$$
\lim _{n \rightarrow \infty} p\left(T_{5}^{9} ; \vec{C}_{3^{n}}^{3}\right)=\lim _{n \rightarrow \infty} \frac{F(n)}{\left(3^{n}\right)_{5}} \cdot 5 !=\frac{3}{8} .
$$

Finalmente, provamos o limitante inferior para $T_{5}^{8}$ no Teorema 4.2.1.

Lema 4.4.4. Temos

$$
\lim _{n \rightarrow \infty} \mathbb{E}\left[p\left(T_{5}^{8} ; \boldsymbol{R}_{\boldsymbol{n}, \mathbf{1 / 2}}\right)\right]=\frac{15}{128}
$$

Demonstração. Pela definição de $\boldsymbol{R}_{\boldsymbol{n}, \mathbf{1} / \mathbf{2}}$, segue que

$$
\mathbb{E}\left[t_{\text {ind }}\left(T_{5}^{8} ; \boldsymbol{R}_{\boldsymbol{n}, \mathbf{1} / \mathbf{2}}\right)\right]=\frac{1}{2^{10}},
$$

para todo $n \geq 5$, assim

$$
\lim _{n \rightarrow \infty} \mathbb{E}\left[p\left(T_{5}^{8} ; \boldsymbol{R}_{\boldsymbol{n}, \mathbf{1 / 2}}\right)\right]=\frac{1}{2^{10}} \cdot 5 !=\frac{15}{128} .
$$

\subsection{Limitantes superiores}

Nesta seção, provamos os limitantes superiores nos Teoremas 4.2.2, 4.2.1 e 4.2.3. Usamos o método semidefinido da álgebra de flags como apresentado na Seção 4.3.

Lema 4.5.1. Para todo torneio $T_{n}$ com $n$ vértices,

$$
\lim _{n \rightarrow \infty} p\left(T_{5}^{12} ; T_{n}\right) \leq \frac{1}{16}
$$


Demonstração. Vamos usar o método semidefinido construindo um programa semidefinido como em (4.5). Para utilizar o método semidefinido, precisamos fixar $\ell$, que será usado para definir o conjunto $\mathcal{T}_{\ell}$. Então precisamos definir $c\left(T ; T^{\prime}\right)$ para todo $T^{\prime} \in \mathcal{T}_{\ell}$. Para definir $c\left(T ; T^{\prime}\right)$, escolhemos quantos tipos $m$ usaremos e os tipos $\sigma_{t}$, para $t \in[m]$, que desejamos utilizar. Para cada tipo $\sigma_{t}$, escolhemos um inteiro $\ell_{t}$ satisfazendo $\ell_{t} \leq\left(\ell+\left|\sigma_{t}\right|\right) / 2$ e uma matriz $\left|\mathcal{F}_{\ell_{t}}^{\sigma_{t}}\right| \times\left|\mathcal{F}_{\ell_{t}}^{\sigma_{t}}\right|$ positiva semidefinida $Q\left(T, \sigma_{t}\right)$.

Fixe $m=3, \ell=5, \ell_{1}=3, \ell_{2}=\ell_{3}=4$ e sejam $\sigma_{1}=1, \sigma_{2}=\operatorname{Tr}_{3}^{*}$ e $\sigma_{3}=\vec{C}_{3}^{*}$ tipos como definidos na Seção 4.3 .4 (veja Figura 4.2).

Sejam $Q\left(T_{5}^{12}, 1\right), Q\left(T_{5}^{12}, \operatorname{Tr}_{3}^{*}\right)$ e $Q\left(T_{5}^{12}, \vec{C}_{3}^{*}\right)$ as matrizes positivas semidefinidas de ordens $\mathcal{F}_{3}^{1} \times \mathcal{F}_{3}^{1}, \mathcal{F}_{4}^{\operatorname{Tr}_{3}^{*}} \times \mathcal{F}_{4}^{\operatorname{Tr}_{3}^{*}}$ e $\mathcal{F}_{4}^{\vec{C}_{3}^{*}} \times \mathcal{F}_{4}^{\vec{C}_{3}^{*}}$ respectivamente apresentadas no Apêndice A (observe que $\left|\mathcal{F}_{3}^{1}\right|=4$ e $\left.\left|\mathcal{F}_{4}^{\operatorname{Tr}_{3}^{*}}\right|=\left|\mathcal{F}_{4}^{\vec{C}_{3}^{*}}\right|=8\right)$.

Para ver que $Q\left(T_{5}^{12}, 1\right), Q\left(T_{5}^{12}, \operatorname{Tr}_{3}^{*}\right)$ e $Q\left(T_{5}^{12}, \vec{C}_{3}^{*}\right)$ são positivas semidefinidas, analisamos seus polinômios característicos $p_{Q\left(T_{5}^{12}, 1\right)}(x), p_{Q\left(T_{5}^{12}, \operatorname{Tr}_{3}^{*}\right)}(x)$ e $p_{Q\left(T_{5}^{12}, \vec{C}_{3}^{*}\right)}(x)$ apresentados no Apêndice B. Como somente os coeficientes de ordem ímpar destes polinômios são negativos, então segue que todas suas raízes são não negativas e, consequentemente, as matrizes são positivas semidefinidas.

Então, computamos $p\left(T_{5}^{12}, T\right)$ e $c\left(Q\left(T_{5}^{12}, \sigma_{t}\right) ; T\right)$ para todo $T \in \mathcal{T}_{5}$ (veja Figura 4.3) e todo $t \in[3]$.

Finalmente, pela Proposição 4.3.10, temos

$$
\lim _{n \rightarrow \infty} p\left(T_{5}^{12} ; T_{n}\right) \leq \max _{T \in \mathcal{T}_{5}}\left\{p\left(T_{5}^{12} ; T\right)+c\left(T_{5}^{12} ; T\right)\right\}=\frac{1}{16},
$$

onde $c\left(T_{5}^{12} ; T\right)=c\left(Q\left(T_{5}^{12}, 1\right) ; T\right)+c\left(Q\left(T_{5}^{12}, \operatorname{Tr}_{3}^{*}\right) ; T\right)+c\left(Q\left(T_{5}^{12}, \vec{C}_{3}^{*}\right) ; T\right)$ para todo $T \in \mathcal{T}_{5}$.

Observação 4.5.2. Todas as matrizes no Apêndice A foram encontradas com o auxílio dos resolvedores de programas semidefinidos CSDP [Bor99] e SDPA [YFF+ 12]. Ademais, as soluções providas por estes resolvedores foram arredondadas para uma solução exata usando o método de arredondamento descrito por Baber [Bab11].

Finalmente, os polinômios característicos no Apêndice $B$ foram encontrados com o auxílio do programa de matemática simbólica Maxima [Max14].

As provas dos limitantes superiores para $T_{5}^{7}, T_{5}^{8}, T_{5}^{9}$ e $T_{5}^{11}$ são muito similares a prova do Lema 4.5.1. Escolhemos quantos tipos $m$ usaremos e quais os tipos $\sigma_{i}$ queremos utilizar. Para 
cada tipo $\sigma_{i}$ escolhemos um inteiro $\ell_{i}$ satisfazendo $\ell_{i} \leq\left(\ell+\left|\sigma_{i}\right|\right) / 2$ e encontramos a matriz positiva semidefinida $Q_{i}=Q\left(T_{5}^{j}, \sigma_{i}\right)$.

As matrizes e seus respectivos polinômios característicos estão apresentados no Apêndice A. Como na prova do Lema 4.5.1, as matrizes são facilmente observadas serem positivas semidefinidas já que os coeficientes negativos dos seus polinômios característicos são somente os de ordem ímpar.

Para cada $j \in\{7,8,9,11,12\}$, computamos $c\left(T_{5}^{j} ; T\right)=\sum_{i=i}^{m} c\left(Q\left(T_{5}^{j}, \sigma_{i}\right) ; T\right)$ e $p\left(T_{5}^{j} ; T\right)$, para todo $T \in \mathcal{T}_{\ell}$, e obtemos os limitantes desejados de acordo com as seguintes tabelas.

\begin{tabular}{|c|c|c|c|c|c|c|c|c|c|}
\hline$T_{5}^{7}$ & & $T_{5}^{8}$ & & $T_{5}^{9}$ & & $T_{5}^{11}$ & & $T_{5}^{12}$ & \\
\hline$m$ & 3 & $m$ & 4 & $m$ & 2 & $m$ & 2 & $m$ & 3 \\
\hline$\sigma_{1}$ & 1 & $\sigma_{1}$ & 1 & $\sigma_{1}$ & 1 & $\sigma_{1}$ & 1 & $\sigma_{1}$ & 1 \\
\hline$\sigma_{2}$ & $\operatorname{Tr}_{3}^{*}$ & $\sigma_{2}$ & $A$ & $\sigma_{2}$ & $\vec{C}_{3}^{*}$ & $\sigma_{2}$ & $\vec{C}_{3}^{*}$ & $\sigma_{2}$ & $\operatorname{Tr}_{3}^{*}$ \\
\hline$\sigma_{3}$ & $\vec{C}_{3}^{*}$ & $\sigma_{3}$ & $\operatorname{Tr}_{3}^{*}$ & $\ell$ & 5 & $\ell$ & 5 & $\sigma_{3}$ & $\vec{C}_{3}^{*}$ \\
\hline$\ell$ & 5 & $\sigma_{4}$ & $\vec{C}_{3}^{*}$ & $\ell_{1}$ & 3 & $\ell_{1}$ & 3 & $\ell$ & 5 \\
\hline$\ell_{1}$ & 3 & $\ell$ & 5 & $\ell_{2}$ & 4 & $\ell_{2}$ & 4 & $\ell_{1}$ & 3 \\
\hline$\ell_{2}$ & 4 & $\ell_{1}$ & 3 & $Q_{1}$ & $Q\left(T_{5}^{9}, 1\right)$ & $Q_{1}$ & $Q\left(T_{5}^{11}, 1\right)$ & $\ell_{2}$ & 4 \\
\hline$\ell_{3}$ & 4 & $\ell_{2}$ & 3 & $Q_{2}$ & $Q\left(T_{5}^{9}, \vec{C}_{3}^{*}\right)$ & $Q_{2}$ & $Q\left(T_{5}^{11}, \vec{C}_{3}^{*}\right)$ & $\ell_{3}$ & 4 \\
\hline$Q_{1}$ & $Q\left(T_{5}^{7}, 1\right)$ & $\ell_{3}$ & 4 & & & & & $Q_{1}$ & $Q\left(T_{5}^{12}, 1\right)$ \\
\hline$Q_{2}$ & $Q\left(T_{5}^{7}, \operatorname{Tr}_{3}^{*}\right)$ & $\ell_{4}$ & 4 & & & & & $Q_{2}$ & $Q\left(T_{5}^{12}, \operatorname{Tr}_{3}^{*}\right)$ \\
\hline$Q_{3}$ & $Q\left(T_{5}^{7}, \vec{C}_{3}^{*}\right)$ & $Q_{1}$ & $Q\left(T_{5}^{8}, 1\right)$ & & & & & $Q_{3}$ & $Q\left(T_{5}^{12}, \vec{C}_{3}^{*}\right)$ \\
\hline & & $Q_{2}$ & $Q\left(T_{5}^{8}, A\right)$ & & & & & & \\
\hline & & $Q_{3}$ & $Q\left(T_{5}^{8}, \operatorname{Tr}_{3}^{*}\right)$ & & & & & & \\
\hline & & $Q_{4}$ & $Q\left(T_{5}^{8}, \vec{C}_{3}^{*}\right)$ & & & & & & \\
\hline
\end{tabular}

\subsection{Unicidade}

Nesta seção, provaremos resultados de unicidade das famílias extremais. Provamos que um homomorfismo $\phi \in \operatorname{Hom}^{+}\left(\mathcal{A}^{0}, \mathbb{R}\right)$ maximiza a densidade de $T_{5}^{8}$ se, e somente se, $\phi$ é o homomorfismo quase-aleatório $\phi_{\mathrm{qr}}$. Também provamos que $\phi \in \operatorname{Hom}^{+}\left(\mathcal{A}^{0}, \mathbb{R}\right)$ maximiza a densidade de $T_{5}^{7}$ ou de $T_{5}^{12}\left(R_{5}\right)$ se, e somente se, $\phi$ é o homomorfismo carrossel $\phi_{\mathrm{R}}$. Por fim, também provamos que $\phi \in \operatorname{Hom}^{+}\left(\mathcal{A}^{0}, \mathbb{R}\right)$ maximiza a densidade de $T_{5}^{9}$ ou de $T_{5}^{11}$ se, e somente se, $\phi$ é o limite da sequência $\left(\vec{C}_{n}^{3}\right)_{n \in \mathbb{N}}$. 


\subsubsection{Unicidade quase-aleatória}

Primeiro vamos relembrar que o homomorfismo quase-aleatório $\phi_{\mathrm{qr}} \in \operatorname{Hom}^{+}\left(\mathcal{A}^{0}, \mathbb{R}\right)$ é quase certamente o limite da sequência de torneios aleatórios $\left(\boldsymbol{R}_{\boldsymbol{n}, \mathbf{1 / 2}}\right)_{n \in \mathbb{N}}$, onde $\boldsymbol{R}_{\boldsymbol{n}, \mathbf{1 / 2}}$ é o torneio aleatório de tamanho $n$ onde cada orientação de um arco é apresentada com probabilidade 1/2 independentemente para todos os pares de vértices.

Alternativamente, o homomorfismo quase-aleatório é definido por

$$
\phi_{\mathrm{qr}}(T)=\frac{\ell !}{|\operatorname{Aut}(T)| 2^{\left(\begin{array}{c}
\ell \\
2
\end{array}\right)}},
$$

para todo torneio $T$ de tamanho $\ell \in \mathbb{N}$, onde $\operatorname{Aut}(T)$ denota o grupo de automorfismos do torneio $T$.

Vamos relembrar também a seguinte equivalência entre as propriedade quase-aleatorias no contexto de homomorfismos positivos.

Lema 4.6.1 (Chung-Graham [CG91, Teorema 1]). Seja $\phi \in \operatorname{Hom}^{+}\left(\mathcal{A}^{0}, \mathbb{R}\right)$ um homomorfismo. Os seguintes são equivalentes.

$$
\begin{aligned}
& P_{1}: \phi=\phi_{\mathrm{qr}} ; \\
& P_{4}: \phi^{\boldsymbol{A}}\left(O^{A}+I^{A}\right)=1 / 2 \text { quase certamente (q.c.). }
\end{aligned}
$$

A definição de $\phi^{\boldsymbol{A}}$ pode ser encontrada no Teorema 4.3 .12 e para a definição das $A$-flags $O^{A}$ e $I^{A}$ veja a Figura 4.2).

Observação 4.6.2. Embora usemos somente duas propriedades de quase-aleatoriedade, vale mencionar que Chung e Graham provaram a equivalência entre um total de 11 propriedades quase-aleatórias ( $P_{1}$ até $\left.P_{11}\right)$.

Agora temos condições de provar que a densidade de $T_{5}^{8}$ é maximizada somente pelo homomorfismo quase-aleatório.

Teorema 4.6.3. Se $\phi \in \operatorname{Hom}^{+}\left(\mathcal{A}^{0}, \mathbb{R}\right)$ é um homomorfismo, então

$$
\phi\left(T_{5}^{8}\right) \leq \frac{15}{128}
$$

com igualdade se, e somente se, $\phi=\phi_{\mathrm{qr}}$. 
Demonstração. Pelo Lema 4.4.4 e pela Proposição 4.3 .10 (veja também a Seção 4.5), sabemos que

$$
\max \left\{\phi\left(T_{5}^{8}\right): \phi \in \operatorname{Hom}^{+}\left(\mathcal{A}^{0}, \mathbb{R}\right)\right\}=\frac{15}{128}=\phi_{\mathrm{qr}}\left(T_{5}^{8}\right)
$$

Ademais, sabemos que as matrizes $Q\left(T_{5}^{8}, 1\right), Q\left(T_{5}^{8}, A\right), Q\left(T_{5}^{8}, \operatorname{Tr}_{3}^{*}\right)$ e $Q\left(T_{5}^{8}, \vec{C}_{3}^{*}\right)$ obtidas pelo método semidefinido formam uma solução ótima com valor 15/128.

Como

$$
Q\left(T_{5}^{8}, A\right)=\frac{99}{3200} v v^{\top}
$$

onde $v=(1,-1,-1,1)$ (indexado por $\left(I^{A}, \vec{C}_{3}^{A}, \operatorname{Tr}_{3}^{A}, O^{A}\right)$ ). Temos que a Proposição 4.3.13, com $F^{\prime}=1_{A}$, implica que, se $\phi \in \operatorname{Hom}^{+}\left(\mathcal{A}^{0}, \mathbb{R}\right)$ é tal que $\phi\left(T_{5}^{8}\right)=15 / 128$, então

$\phi^{\boldsymbol{A}}(F(v))=\boldsymbol{\phi}^{\boldsymbol{A}}\left(I^{A}-\vec{C}_{3}^{A}-\operatorname{Tr}_{3}^{A}+O^{A}\right)=0$ q.c. $\quad$ (Para a definição de $F(v)$, veja a Seção 4.3.2)

Uma vez que $\vec{C}_{3}^{A}+\operatorname{Tr}_{3}^{A}=1_{A}-O^{A}-I^{A}$, temos

$$
\phi^{A}\left(O^{A}+I^{A}\right)=\frac{1}{2} \text { q.c. }
$$

consequentemente $\phi$ satisfaz a Propriedade $P_{4}$ do Lema 4.6.1. Portanto $\phi=\phi_{\mathrm{qr}}$.

\subsubsection{Unicidade quase-carrossel}

Primeiro vamos relembrar a definição do homomorfismo carrossel $\phi_{\mathrm{R}} \in \operatorname{Hom}^{+}\left(\mathcal{A}^{0}, \mathbb{R}\right)$ como o limite da sequência $\left(R_{2 n+1}\right)_{n \in \mathbb{N}}$ dos torneios carrossel (veja a definição na Seção 2.2). Analogamente às propriedades quase-aleatórias, as propriedades quase-carrossel [Cor15] são propriedades de equivalência sobre os homomorfismos $\phi \in \operatorname{Hom}^{+}\left(\mathcal{A}^{0}, \mathbb{R}\right)$ que forçam $\phi=\phi_{\mathrm{R}}$. Analogamente ao torneio localmente transitivo, um homomorfismo $\phi \in \operatorname{Hom}^{+}\left(\mathcal{A}^{0}, \mathbb{R}\right)$ satisfazendo $\phi\left(W_{4}+L_{4}\right)=0$ é chamado de localmente transitivo. Possivelmente a propriedade quase-carrossel mais importante afirma que $\phi_{\mathrm{R}}$ é o único homomorfismo que é balanceado e localmente transitivo.

Vamos recordar duas propriedades quase-carrossel abaixo. Para as flags e tipos utilizados, veja a Figura 4.2 
Lema 4.6.4 ([Cor15, Lema 3.2]). Seja $\phi \in \operatorname{Hom}^{+}\left(\mathcal{A}^{0}, \mathbb{R}\right)$ um homomorfismo. Os seguintes são equivalentes.

$S_{1}: \phi=\phi_{\mathrm{R}} ;$

$S_{2}: \phi$ é balanceado e localmente transitivo, isto é, temos

$$
\phi^{\mathbf{1}}(\alpha)=\phi^{\mathbf{1}}(\beta) \text { q.c.; } \quad \phi\left(W_{4}+L_{4}\right)=0 .
$$

Ademais, precisamos de uma equivalência com relação aos homomorfismos balanceados.

Lema 4.6.5 (Chung-Graham [CG91, Teorema 2]). Seja $\phi \in \operatorname{Hom}^{+}\left(\mathcal{A}^{0}, \mathbb{R}\right)$ um homomorfismo. Os seguintes são equivalentes.

$$
Q_{1}: \phi\left(\operatorname{Tr}_{3}\right)=3 / 4 \text { e } \phi\left(\vec{C}_{3}\right)=1 / 4
$$

$Q_{4}: \phi$ é balanceado, isto é, temos $\phi^{\mathbf{1}}(\alpha)=\phi^{\mathbf{1}}(\beta)$ q.c.

Analogamente ao Teorema 4.6.3, a unicidade para o homomorfismo carrossel seguirá da Propriedade $S_{2}$.

Teorema 4.6.6. Se $\phi \in \operatorname{Hom}^{+}\left(\mathcal{A}^{0}, \mathbb{R}\right)$ é um homomorfismo, então

$$
\phi\left(T_{5}^{7}\right) \leq \frac{5}{16}
$$

com igualdade se, e somente se, $\phi=\phi_{\mathrm{R}}$.

Demonstração. Pelo Lema 4.4.2 e pela Proposição 4.3 .10 (veja também a Seção 4.5), sabemos que

$$
\max \left\{\phi\left(T_{5}^{7}\right): \phi \in \operatorname{Hom}^{+}\left(\mathcal{A}^{0}, \mathbb{R}\right)\right\}=\frac{5}{16}=\phi_{\mathrm{R}}\left(T_{5}^{7}\right)
$$

Nosso objeto é provar que todo $\phi \in \operatorname{Hom}^{+}\left(\mathcal{A}^{0}, \mathbb{R}\right)$ tal que $\phi\left(T_{5}^{7}\right)=5 / 16$ é balanceado e localmente transitivo.

Para provar que tal $\phi$ é balanceado, observamos que as matrizes $Q\left(T_{5}^{7}, 1\right), Q\left(T_{5}^{7}, \operatorname{Tr}_{3}^{*}\right)$ e $Q\left(T_{5}^{7}, \vec{C}_{3}^{*}\right)$ obtidas do método semidefinido são uma solução ótima com valor $5 / 16$, e como 
temos que

$$
Q\left(T_{5}^{7}, 1\right)=\frac{35}{48} v v^{\top}
$$

onde $v=(1,-1,-1,1)$ (indexado por $\left(\operatorname{Tr}_{3}^{1, L}, \vec{C}_{3}^{1}, \operatorname{Tr}_{3}^{1, M}, \operatorname{Tr}_{3}^{1, W}\right)$ ), temos que a Proposição 4.3.13, com $F^{\prime}=1_{1}$, implica que

$$
\boldsymbol{\phi}^{\mathbf{1}}(F(v))=\boldsymbol{\phi}^{\mathbf{1}}\left(\operatorname{Tr}_{3}^{1, L}-\vec{C}_{3}^{1}-\operatorname{Tr}_{3}^{1, M}+\operatorname{Tr}_{3}^{1, W}\right)=0 \text { q.c. }
$$

Pela definição do produto da álgebra na Proposição 4.3.2, temos que $(\alpha-\beta)^{2}=\operatorname{Tr}_{3}^{1, L}-\vec{C}_{3}^{1}-$ $\operatorname{Tr}_{3}^{1, M}+\operatorname{Tr}_{3}^{1, W}$. Ademais, temos que $\phi^{\mathbf{1}}\left((\alpha-\beta)^{2}\right)=0$ q.c. Portanto $\phi^{\mathbf{1}}(\alpha)=\boldsymbol{\phi}^{\mathbf{1}}(\beta)$ q.c., isto é, o homomorfismo $\phi$ é balanceado.

Para provar que $\phi$ é também localmente transitivo, usaremos a Proposição 4.3.11. A Tabela 4.1 tem os valores de $p\left(T_{5}^{7}+g ; T^{\prime}\right)$ para $T^{\prime} \in \mathcal{T}_{5}$ e onde

$$
\begin{array}{c|cccccccccccc}
\multicolumn{8}{c}{g=\llbracket F\left(Q\left(T_{5}^{7}, 1\right)\right) \rrbracket_{1}+\llbracket F\left(Q\left(T_{5}^{7}, \operatorname{Tr}_{3}^{*}\right)\right) \rrbracket_{\operatorname{Tr}_{3}^{*}}+\llbracket F\left(Q\left(T_{5}^{7}, \vec{C}_{3}^{*}\right)\right) \rrbracket_{\vec{C}_{3}^{*} \cdot}} \\
T^{\prime} & T_{5}^{1} & T_{5}^{2} & T_{5}^{3} & T_{5}^{4} & T_{5}^{5} & T_{5}^{6} & T_{5}^{7} & T_{5}^{8} & T_{5}^{9} & T_{5}^{10} & T_{5}^{11} & T_{5}^{12} \\
\hline p\left(T_{5}^{7}+g ; T^{\prime}\right) & \frac{5}{16} & -\frac{7}{80} & \frac{11}{48} & -\frac{29}{240} & -\frac{7}{80} & \frac{11}{48} & \frac{5}{16} & -\frac{13}{48} & \frac{5}{16} & \frac{1}{16} & -\frac{109}{240} & \frac{5}{16}
\end{array}
$$

Tabela 4.1: Valores $p\left(T_{5}^{7}+g ; T^{\prime}\right)$ para $T^{\prime} \in \mathcal{T}_{5}$ onde $g=\llbracket F\left(Q\left(T_{5}^{7}, 1\right)\right) \rrbracket_{1}+\llbracket F\left(Q\left(T_{5}^{7}, \operatorname{Tr}_{3}^{*}\right)\right) \rrbracket_{\operatorname{Tr}_{3}^{*}}+$ $\llbracket F\left(Q\left(T_{5}^{7}, \vec{C}_{3}^{*}\right)\right) \rrbracket_{\vec{C}_{3}^{*}}$.

A Proposição 4.3.11 implica que se $\phi\left(T^{\prime}\right)>0$ para $T^{\prime} \in \mathcal{T}_{5}$, então $T^{\prime} \in\left\{T_{5}^{1}, T_{5}^{7}, T_{5}^{9}, T_{5}^{12}\right\}$, e como temos que estes quatro torneios são os únicos torneios localmente transitivos de tamanho 5 (i.e., os únicos torneios $T^{\prime} \in \mathcal{T}_{5} \operatorname{com} p\left(W_{4}+L_{4} ; T^{\prime}\right)=0$ ), temos $\phi\left(W_{4}+L_{4}\right)=0$, isto é, o homomorfismo $\phi$ é localmente transitivo.

Portanto $\phi$ satisfaz a Propriedade quase-carrossel $S_{2}$, consequentemente $\phi=\phi_{\mathrm{R}}$ pelo Lema 4.6.4.

Teorema 4.6.7. Se $\phi \in \operatorname{Hom}^{+}\left(\mathcal{A}^{0}, \mathbb{R}\right)$ é um homomorfismo, então

$$
\phi\left(T_{5}^{12}\right) \leq \frac{1}{16},
$$


com igualdade se, e somente se, $\phi=\phi_{\mathrm{R}}$.

Demonstração. Pelo Lema 4.4.2 e pela Proposição 4.3 .10 (veja também a Seção 4.5), temos que

$$
\max \left\{\phi\left(T_{5}^{12}\right): \phi \in \operatorname{Hom}^{+}\left(\mathcal{A}^{0}, \mathbb{R}\right)\right\}=\frac{1}{16}=\phi_{\mathrm{R}}\left(T_{5}^{12}\right)
$$

Novamente nosso objeto é mostrar que todo $\phi \in \operatorname{Hom}^{+}\left(\mathcal{A}^{0}, \mathbb{R}\right)$ tal que $\phi\left(T_{5}^{12}\right)=1 / 16$ é balanceado e localmente transitivo.

Para provar que tal $\phi$ é balanceado, observe que as matrizes $Q\left(T_{5}^{12}, 1\right), Q\left(T_{5}^{12}, \operatorname{Tr}_{3}^{*}\right)$ e $Q\left(T_{5}^{12}, \vec{C}_{3}^{*}\right)$ obtidas pelo método semidefinido são uma solução ótima com valor $1 / 16$, e consequentemente

$$
Q\left(T_{5}^{12}, 1\right)=\frac{1}{16} v v^{\top}
$$

onde $v=(1,-1,-1,1)$ (indexado por $\left.\left(\operatorname{Tr}_{3}^{1, L}, \vec{C}_{3}^{1}, \operatorname{Tr}_{3}^{1, M}, \operatorname{Tr}_{3}^{1, W}\right)\right)$. Temos que a Proposição 4.3.13, com $F^{\prime}=1_{1}$, implica que

$$
\phi^{\mathbf{1}}(F(v))=\boldsymbol{\phi}^{\mathbf{1}}\left(\operatorname{Tr}_{3}^{1, L}-\vec{C}_{3}^{1}-\operatorname{Tr}_{3}^{1, M}+\operatorname{Tr}_{3}^{1, W}\right)=0 \text { q.c. }
$$

Pela definição do produto da álgebra na Proposição 4.3.2, temos que $(\alpha-\beta)^{2}=\operatorname{Tr}_{3}^{1, L}-\vec{C}_{3}^{1}-$ $\operatorname{Tr}_{3}^{1, M}+\operatorname{Tr}_{3}^{1, W}$. Ademais, temos que $\phi^{\mathbf{1}}\left((\alpha-\beta)^{2}\right)=0$ q.c. Portanto, $\phi^{\mathbf{1}}(\alpha)=\boldsymbol{\phi}^{\mathbf{1}}(\beta)$ q.c., isto é, o homomorfismo $\phi$ é balanceado.

Para provar que $\phi$ é também localmente transitivo, usaremos novamente a Proposição 4.3.11.

A Tabela 4.2 tem os valores de $p\left(T_{5}^{12}+g ; T^{\prime}\right)$ para $T^{\prime} \in \mathcal{T}_{5}$ onde

$$
\begin{array}{c|cccccccccccc}
g=\llbracket F\left(Q\left(T_{5}^{12}, 1\right)\right) \rrbracket_{1}+\llbracket F\left(Q\left(T_{5}^{12}, \operatorname{Tr}_{3}^{*}\right)\right) \rrbracket_{\operatorname{Tr}_{3}^{*}}+\llbracket F\left(Q\left(T_{5}^{12}, \vec{C}_{3}^{*}\right)\right) \rrbracket_{\vec{C}_{3}^{*}} \cdot \\
T^{\prime} & T_{5}^{1} & T_{5}^{2} & T_{5}^{3} & T_{5}^{4} & T_{5}^{5} & T_{5}^{6} & T_{5}^{7} & T_{5}^{8} & T_{5}^{9} & T_{5}^{10} & T_{5}^{11} & T_{5}^{12} \\
\hline p\left(T_{5}^{12}+g ; T^{\prime}\right) & \frac{1}{16} & \frac{1}{80} & \frac{1}{16} & -\frac{3}{16} & \frac{1}{80} & \frac{1}{16} & \frac{1}{16} & \frac{1}{16} & \frac{1}{16} & \frac{1}{16} & -\frac{39}{80} & \frac{1}{16}
\end{array}
$$

Tabela 4.2: Valores $p\left(T_{5}^{12}+g ; T^{\prime}\right)$ para $T^{\prime} \in \mathcal{T}_{5}$ onde $g=\llbracket F\left(Q\left(T_{5}^{12}, 1\right)\right) \rrbracket_{1}+\llbracket F\left(Q\left(T_{5}^{12}, \operatorname{Tr}_{3}^{*}\right)\right) \rrbracket_{\operatorname{Tr}_{3}^{*}}+$ $\llbracket F\left(Q\left(T_{5}^{12}, \vec{C}_{3}^{*}\right)\right) \rrbracket_{\vec{C}_{3}^{*}}$.

A Proposição 4.3.11 implica que $\phi\left(T_{5}^{2}+T_{5}^{5}\right)=0$. 
Agora, como temos

$$
\llbracket\left(L_{4}^{\vec{C}_{3}^{*}}\right)^{2} \rrbracket_{\vec{C}_{3}^{*}}=\frac{1}{20} T_{5}^{2} ; \quad \llbracket\left(W_{4}^{\vec{C}_{3}^{*}}\right)^{2} \rrbracket_{\vec{C}_{3}^{*}}=\frac{1}{20} T_{5}^{5}
$$

e uma vez que $\phi$ é balanceado, pelo Lema 4.6 .5 , temos $\phi\left(\vec{C}_{3}\right)=1 / 4$, consequentemente

$$
\mathbb{E}\left[\phi^{\vec{C}_{3}^{*}}\left(W_{4}^{\vec{C}_{3}^{*}}\right)^{2}+\phi^{\vec{C}_{3}^{*}}\left(L_{4}^{\vec{C}_{3}^{*}}\right)^{2}\right]=\frac{1}{10} \cdot \frac{\phi\left(T_{5}^{2}+T_{5}^{5}\right)}{\phi\left(\vec{C}_{3}\right)}=0
$$

o que implica que $\boldsymbol{\phi}^{\vec{C}_{3}^{*}}\left(W_{4}^{\vec{C}_{3}^{*}}+L_{4}^{\vec{C}_{3}^{*}}\right)=0$ q.c..

Isto por sua vez implica que

$$
0=\mathbb{E}\left[\phi^{\vec{C}_{3}^{*}}\left(W_{4}^{\vec{C}_{3}^{*}}+L_{4}^{\vec{C}_{3}^{*}}\right)\right]=\frac{1}{4} \cdot \frac{\phi\left(W_{4}+L_{4}\right)}{\phi\left(\vec{C}_{3}\right)}
$$

consequentemente $\phi\left(W_{4}+L_{4}\right)=0$, isto é, o homomorfismo $\phi$ é localmente transitivo.

Portanto $\phi$ satisfaz a Propriedade quase-carrossel $S_{2}$, com isso $\phi=\phi_{\mathrm{R}}$ pelo Lema 4.6.4.

\subsubsection{Unicidade quase-triangular}

Começaremos definindo um torneio $\vec{C}_{3}$-decomponível indutivamente. Intuitivamente, um torneio $\vec{C}_{3}$-decomponível tem estrutura similar ao $\vec{C}_{n}^{3}$ (veja definição na Seção 2.3), mas não requer que o "blow-up" tenha partes balanceadas, i.e., as partes podem ter tamanhos distintos.

Definição 4.6.8. Defina a sequência de conjuntos $\left(\mathcal{B}_{n}\right)_{n \in \mathbb{N}}$ indutivamente como segue.

Sejam $\mathcal{B}_{0}=\mathcal{T}_{0}$ e $\mathcal{B}_{1}=\mathcal{T}_{1}$ e, para $n \geq 2$, seja $\mathcal{B}_{n} \subset \mathcal{T}_{n}$ o conjunto de todos os torneios com tamanho $n$ tais que existem conjuntos $A, B$ e $C$ tais que

i. Os conjuntos $A, B$ e $C$ são estritamente contidos em $V(T)$, i.e., temos $A, B, C \subsetneq V(T)$;

ii. Os conjuntos $A, B$ e $C$ são disjuntos dois a dois;

iii. Temos $V(T)=A \cup B \cup C$;

iv. Temos $\left.T\right|_{A} \in \mathcal{B}_{|A|},\left.T\right|_{B} \in \mathcal{B}_{|B|}$ e $\left.T\right|_{C} \in \mathcal{B}_{|C|}$;

v. Temos $A \times B, B \times C, C \times A \subset A(T)$.

Finalmente, dizemos que um torneio $T$ de tamanho $n$ é $\vec{C}_{3}$-decomponível se $T \in \mathcal{B}_{n}$. 
Observação 4.6.9. Observe que os itens (i), (ii) e (iii) juntos afirmam que $\{A, B, C\} \backslash\{\varnothing\}$ é uma partição de $V(T)$ em dois ou três conjuntos. Ademais, observe que o item (ii) na realidade segue do item (v). Finalmente, observe que o item (iv) é bem definido já que $\max \{|A|,|B|,|C|\}<n$ (devido ao item (i)).

O próximo teorema provê uma caracterização do teorema de $\vec{C}_{3}$-decomponibilidade como a classe dos torneios que não contêm $T_{5}^{8}, T_{5}^{10}$ e $T_{5}^{12}$. Adiaremos a prova desse teorema para a Seção 4.6.4.

Teorema 4.6.10. Um torneio $T$ é $\vec{C}_{3}$-decomponível se, e somente, se não contém cópias de $T_{5}^{8}$, $T_{5}^{10}$ nem de $T_{5}^{12}$.

Motivado pelo teorema acima, definimos que um homomorfismo $\phi \in \operatorname{Hom}^{+}\left(\mathcal{A}^{0}, \mathbb{R}\right)$ é $\vec{C}_{3^{-}}$ decomponível se $\phi\left(T_{5}^{8}+T_{5}^{10}+T_{5}^{12}\right)=0$. Observe que o fato de que a sequência de torneios $\left(T_{n}\right)_{n \in \mathbb{N}}$ converge a um homomorfismo $\vec{C}_{3}$-decomponível não implica que todo torneio nessa sequência é $\vec{C}_{3}$-decomponível. Em vez disso somente implica que a densidade dos torneios $T_{5}^{8}$, $T_{5}^{10}$ e $T_{5}^{12}$ em $T_{n}$ vai para zero quando $n$ vai para infinito.

Agora definiremos a noção de um torneio $k$-igualmente $\vec{C}_{3}$-decomponível indutivamente.

Definição 4.6.11. Um torneio $T$ é 0-igualmente $\vec{C}_{3}$-decomponível se este é $\vec{C}_{3}$-decomponível.

Para $k>0$, um torneio $T$ é $k$-igualmente $\vec{C}_{3}$-decomponivel se ou $|T| \leq 1$ ou existem $(A, B, C)$ como na Definição 4.6.8 satisfazendo também as seguintes propriedades.

a. Temos $\max \{|A|,|B|,|C|\}-\min \{|A|,|B|,|C|\} \leq 1$;

b. Os torneios $\left.T\right|_{A},\left.T\right|_{B}$ e $\left.T\right|_{C}$ são $(k-1)$-igualmente $\vec{C}_{3}$-decomponível.

Trivialmente, todo torneio $k$-igualmente $\vec{C}_{3}$-decomponível é também $(k-1)$-igualmente $\vec{C}_{3}$ decomponível.

Observe também que se $n=3^{k}$, então o único torneio com tamanho $n$ que é $k$-igualmente $\vec{C}_{3}$-decomponível é o torneio $\vec{C}_{n}^{3}$. Agora afirmamos que a sequência $\left(\vec{C}_{n}^{3}\right)_{n \in \mathbb{N}}$ é convergente, mas adiaremos a prova dessa afirmação. Chamaremos o limite dessa sequência de homomorfismo triangular e denotamos esse por $\phi_{\vec{C}_{3}}$.

O próximo teorema afirma a equivalência das propriedades que chamaremos de propriedades de quase-triangularidade. A equivalência das Propriedades $L_{1}, L_{2}$ e $L_{3}$ no Teorema 4.6.12 implicam que $\phi_{\vec{C}_{3}}$ é o único homomorfismo que maximiza a densidade de $T_{5}^{9}$ e também a densidade 
de $T_{5}^{11}$.

Teorema 4.6.12. Se $\phi \in \operatorname{Hom}^{+}\left(\mathcal{A}^{0}, \mathbb{R}\right)$ é um homomorfismo, então os seguintes são equivalentes.

$L_{1}: \phi=\phi_{\vec{C}_{3}}$

$L_{2}: \phi$ maximiza a densidade de $T_{5}^{9}$, isto é, temos

$$
\phi\left(T_{5}^{9}\right)=\max \left\{\psi\left(T_{5}^{9}\right): \psi \in \operatorname{Hom}^{+}\left(\mathcal{A}^{0}, \mathbb{R}\right)\right\}
$$

$L_{3}: \phi$ maximiza a densidade de $T_{5}^{11}$, isto é, temos

$$
\phi\left(T_{5}^{11}\right)=\max \left\{\psi\left(T_{5}^{11}\right): \psi \in \operatorname{Hom}^{+}\left(\mathcal{A}^{0}, \mathbb{R}\right)\right\} ;
$$

$L_{4}$ : $\phi$ é balanceado e $\vec{C}_{3}$-decomponível, isto é, temos

$$
\phi^{\mathbf{1}}(\alpha)=\phi^{\mathbf{1}}(\beta) \text { q.c.; } \quad \phi\left(T_{5}^{8}+T_{5}^{10}+T_{5}^{12}\right)=0
$$

$L_{5}:$ Para todo $k \in \mathbb{N}$, existe uma sequência $\left(T_{n}^{(k)}\right)_{n \in \mathbb{N}}$ de torneios $k$-igualmente $\vec{C}_{3}$-decomponíveis que convergem para $\phi$.

Provaremos o Teorema 4.6.12 através de uma série de lemas. Já provamos na Seção 4.4 e 4.5 que $L_{1} \Longrightarrow L_{2} \wedge L_{3}$.

Os próximos dois lemas seguem das técnicas apresentadas na Seção 4.3.3.

Lema 4.6.13. Temos $L_{2} \Longrightarrow L_{4}$.

Demonstração. Pelo Lema 4.4.3 e pela Proposição 4.3 .10 (veja também Seção 4.5), sabemos que

$$
\max \left\{\psi\left(T_{5}^{9}\right): \psi \in \operatorname{Hom}^{+}\left(\mathcal{A}^{0}, \mathbb{R}\right)\right\}=\frac{3}{8},
$$


e que as matrizes $Q\left(T_{5}^{9}, 1\right)$ e $Q\left(T_{5}^{9}, \vec{C}_{3}^{*}\right)$ obtidas pelo método semidefinido formam uma solução ótima com valor $3 / 8$.

Seja então $\phi \in \operatorname{Hom}^{+}\left(\mathcal{A}^{0}, \mathbb{R}\right)$ um homomorfismo que maximiza a densidade de $T_{5}^{9}$. Provaremos que $\phi$ é $\vec{C}_{3}$-decomponível. Para isso, usaremos a Proposição 4.3.11. A Tabela 4.3 contém os valores $p\left(T_{5}^{9}+g ; T^{\prime}\right)$ para $T^{\prime} \in \mathcal{T}_{5}$ e onde

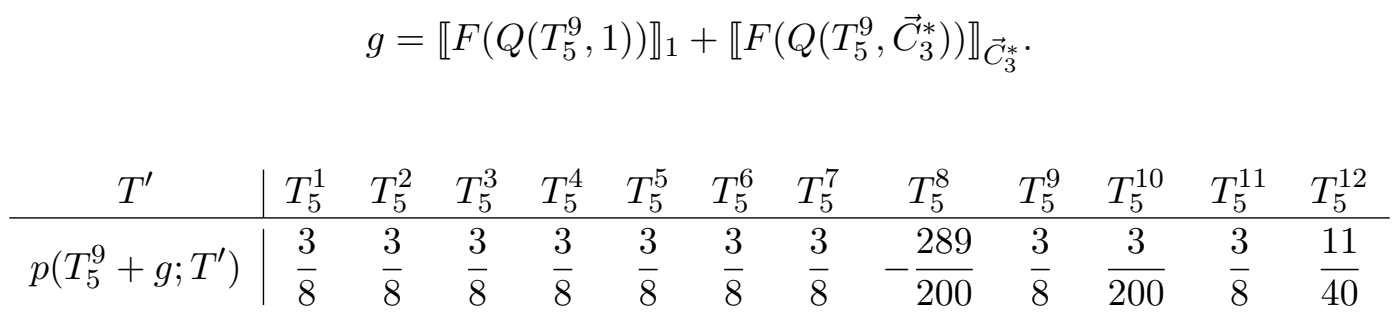

Tabela 4.3: valores $p\left(T_{5}^{9}+g ; T^{\prime}\right)$ para $T^{\prime} \in \mathcal{T}_{5}$ e onde $g=\llbracket F\left(Q\left(T_{5}^{9}, 1\right)\right) \rrbracket_{1}+\llbracket F\left(Q\left(T_{5}^{9}, \vec{C}_{3}^{*}\right)\right) \rrbracket_{\vec{C}_{3}^{*}}$.

A Proposição 4.3.11 implica que $\phi\left(T_{5}^{8}+T_{5}^{10}+T_{5}^{12}\right)=0$, isso é, o homomorfismo $\phi$ é $\vec{C}_{3^{-}}$ decomponível.

Com isso, falta apenas provar que $\phi$ é balanceado.

Primeiro, notemos que a matriz $Q\left(T_{5}^{9}, 1\right)$ tem autovetores

$$
\begin{aligned}
& v_{1}=\left(\begin{array}{llll}
1, & \frac{-16+\sqrt{179}}{7}, & \frac{2-\sqrt{179}}{7}, & 1
\end{array}\right) ; \\
& v_{2}=\left(\begin{array}{lll}
1, & \frac{-16-\sqrt{179}}{7}, \frac{2+\sqrt{179}}{7}, & 1
\end{array}\right)
\end{aligned}
$$

(indexado por $\left.\left(\operatorname{Tr}_{3}^{1, L}, \vec{C}_{3}^{1}, \operatorname{Tr}_{3}^{1, M}, \operatorname{Tr}_{3}^{1, W}\right)\right)$ com os autovalores $12(16+\sqrt{179})$ e $12(16-\sqrt{179})$ respectivamente.

Pela Proposição 4.3.13, com $F^{\prime}=1_{1}$, sabemos que $\phi^{\mathbf{1}}\left(F\left(v_{1}\right)\right)=\phi^{\mathbf{1}}\left(F\left(v_{2}\right)\right)=0$ quase certamente. Como $\mathbb{E}\left[\phi^{\mathbf{1}}\left(F\left(v_{1}\right)-F\left(v_{2}\right)\right)\right]=0$, temos que

$$
\begin{aligned}
\mathbb{E}\left[\phi^{\mathbf{1}}\left(F\left(v_{1}\right)-F\left(v_{2}\right)\right)\right] & =\frac{2 \sqrt{179}}{7} \mathbb{E}\left[\phi^{\mathbf{1}}\left(\vec{C}_{3}^{1}-\operatorname{Tr}_{3}^{1, M}\right)\right] \\
& \left.=\frac{2 \sqrt{179}}{7}\left(\mathbb{E}\left[\phi^{\mathbf{1}}\left(\vec{C}_{3}^{1}\right)\right]-\mathbb{E}\left[\phi^{\mathbf{1}} \operatorname{Tr}_{3}^{1, M}\right)\right]\right) \\
& =\frac{2 \sqrt{179}}{7}\left(\phi\left(\vec{C}_{3}\right)-\frac{1}{3} \phi\left(\operatorname{Tr}_{3}\right)\right) .
\end{aligned}
$$

A Equação (4.7) segue do Teorema 4.3.12. Como $\vec{C}_{3}+\operatorname{Tr}_{3}=1_{0}$, temos que $\phi\left(\vec{C}_{3}\right)=1 / 4$. Desta forma, pelo Lema 4.6.5, implica que $\phi$ é balanceado. 
Portando $\phi$ satisfaz $L_{4}$.

Lema 4.6.14. Temos $L_{3} \Longrightarrow L_{4}$.

Demonstração. Pelo Lema 4.4.3 e pela Proposição 4.3.10 (veja também a Seção 4.5), nos sabemos que

$$
\max \left\{\psi\left(T_{5}^{11}\right): \psi \in \operatorname{Hom}^{+}\left(\mathcal{A}^{0}, \mathbb{R}\right)\right\}=\frac{1}{16},
$$

e que as matrizes $Q\left(T_{5}^{11}, 1\right)$ e $Q\left(T_{5}^{11}, \vec{C}_{3}^{*}\right)$ obtidas pelo método semidefinido formam uma solução ótima com valor $1 / 16$.

Seja então $\phi \in \operatorname{Hom}^{+}\left(\mathcal{A}^{0}, \mathbb{R}\right)$ um homomorfismo que maximiza a densidade de $T_{5}^{11}$. Como

$$
Q\left(T_{5}^{11}, 1\right)=\frac{5}{16} v v^{\top}
$$

onde $v=(1,-1,-1,1)$ (indexado por $\left(\operatorname{Tr}_{3}^{1, L}, \vec{C}_{3}^{1}, \operatorname{Tr}_{3}^{1, M}, \operatorname{Tr}_{3}^{1, W}\right)$ ), temos que a Proposição 4.3.13, $\operatorname{com} F^{\prime}=1_{1}$, implica que

$$
\phi^{\mathbf{1}}(F(v))=\phi^{\mathbf{1}}\left(\operatorname{Tr}_{3}^{1, L}-\vec{C}_{3}^{1}-\operatorname{Tr}_{3}^{1, M}+\operatorname{Tr}_{3}^{1, W}\right)=0 .
$$

Pela definição do produto da álgebra na Proposição 4.3.2, temos que $(\alpha-\beta)^{2}=\operatorname{Tr}_{3}^{1, L}-\vec{C}_{3}^{1}-$ $\operatorname{Tr}_{3}^{1, M}+\operatorname{Tr}_{3}^{1, W}$. Ademais, temos que $\boldsymbol{\phi}^{\mathbf{1}}\left((\alpha-\beta)^{2}\right)=0$ q.c. Entretanto $\boldsymbol{\phi}^{\mathbf{1}}(\alpha)=\boldsymbol{\phi}^{\mathbf{1}}(\beta)$ quase certamente, isto é, o homomorfismo $\phi$ é balanceado.

Só resta provar que $\phi$ é $\vec{C}_{3}$-decomponível. Para fazer isso, usaremos a Proposição 4.3.11. A Tabela 4.4 tem os valores de $p\left(T_{5}^{11}+g ; T^{\prime}\right)$ para $T^{\prime} \in \mathcal{T}_{5}$ e onde

$$
\begin{array}{c|cccccccccccc}
\multicolumn{1}{c}{g=\llbracket F\left(Q\left(T_{5}^{11}, 1\right)\right) \rrbracket_{1}+\llbracket F\left(Q\left(T_{5}^{11}, \vec{C}_{3}^{*}\right)\right) \rrbracket_{\vec{C}_{3}^{*}} \cdot} \\
T^{\prime} & T_{5}^{1} & T_{5}^{2} & T_{5}^{3} & T_{5}^{4} & T_{5}^{5} & T_{5}^{6} & T_{5}^{7} & T_{5}^{8} & T_{5}^{9} & T_{5}^{10} & T_{5}^{11} & T_{5}^{12} \\
\hline p\left(T_{5}^{11}+g ; T^{\prime}\right) & \frac{1}{16} & \frac{1}{16} & \frac{1}{16} & \frac{1}{16} & \frac{1}{16} & \frac{1}{16} & \frac{1}{16} & -\frac{3}{400} & \frac{1}{16} & -\frac{23}{400} & \frac{1}{16} & \frac{1}{80}
\end{array}
$$

Tabela 4.4: Valores $p\left(T_{5}^{11}+g ; T^{\prime}\right)$ para $T^{\prime} \in \mathcal{T}_{5}$ e onde $g=\llbracket F\left(Q\left(T_{5}^{11}, 1\right)\right) \rrbracket_{1}+\llbracket F\left(Q\left(T_{5}^{11}, \vec{C}_{3}^{*}\right)\right) \rrbracket_{\vec{C}_{3}^{*}}$.

A Proposição 4.3.11 implica que $\phi\left(T_{5}^{8}+T_{5}^{10}+T_{5}^{12}\right)=0$, isso é, o homomorfismo $\phi$ é $\vec{C}_{3^{-}}$ 
decomponível. Portanto $\phi$ satisfaz $L_{4}$.

Para as duas próximas implicações, precisaremos usar a noção de uma $\vec{C}_{3}$-decomposição de um torneio $\vec{C}_{3}$-decomponível. Para fazer isso precisamente, vamos primeiramente fixar algumas notações. Denote

$$
\Sigma^{*}=\left\{\left(\sigma_{i}\right)_{i=1}^{k}: k \in \mathbb{N} \text { e } \forall i \in[k], \sigma_{i} \in[3]\right\}
$$

como o conjunto de toda as sequências de elementos em $[3]=\{1,2,3\}$ (e denotemos a sequência vazia por $\epsilon$ ).

Como usual, denotaremos por $\sigma \tau$ a sequência obtida pela concatenação de $\tau \in \Sigma^{*}$ no fim de $\sigma \in \Sigma^{*}$. Observe que para $\tau=1$, temos que $\sigma \tau=\sigma 1$ como na Definição 4.6.15. Denotaremos o tamanho da sequência $\sigma \in \Sigma^{*}$ por $|\sigma|$.

Definição 4.6.15. Seja $T$ um torneio $\vec{C}_{3}$-decomponível. Uma $\vec{C}_{3}$-decomposição do torneio $T$ é uma família de conjuntos $A=\left(A_{\sigma}\right)_{\sigma \in \Sigma^{*}}$ indexada por $\Sigma^{*}$ tal que

i. Temos $A_{\epsilon}=V(T)$;

ii. Para todo $\sigma \in \Sigma^{*}$ tal que $\left|A_{\sigma}\right| \geq 2$, a tripla $\left(A_{\sigma 1}, A_{\sigma 2}, A_{\sigma 3}\right)$ satisfaz os itens na Definição 4.6.8 para $\left.T\right|_{A_{\sigma}}$;

iii. Para todo $\sigma \in \Sigma^{*}$ tal que $\left|A_{\sigma}\right| \leq 1$, os conjuntos $A_{\sigma 1}, A_{\sigma 2}$ e $A_{\sigma 3}$ são dois a dois disjuntos e $A_{\sigma 1} \cup A_{\sigma 2} \cup A_{\sigma 3}=A_{\sigma}$.

Observe que, dado um $\sigma \in \Sigma^{*}$, temos que $A_{\sigma} \subseteq V(T)$. Veja na Figura 4.6 um exemplo de uma $\vec{C}_{3}$-decomposição que estamos apresentando apenas no máximo 2 níveis.

Para todo $k \in \mathbb{N}$, o $k$-ésimo nível da $\vec{C}_{3}$-decomposição $A$ é a família de conjuntos $A_{\sigma}$ tais que $|\sigma|=k$. A assimetria do $k$-ésimo nível de $A$ (denotada por $\Delta_{k}(A)$ ) é definida como

$$
\Delta_{k}(A)=\max \left\{\left|A_{\sigma}\right|: \sigma \in \Sigma^{*} e|\sigma|=k\right\}-\min \left\{\left|A_{\sigma}\right|: \sigma \in \Sigma^{*} e|\sigma|=k\right\}
$$

Observe que um torneio é $k$-igualmente $\vec{C}_{3}$-decomponível se, e somente se, ele tem uma

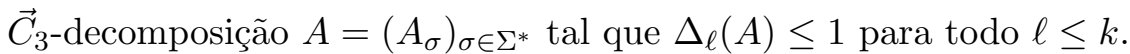

Agora definiremos algumas notações sobre torneios. 


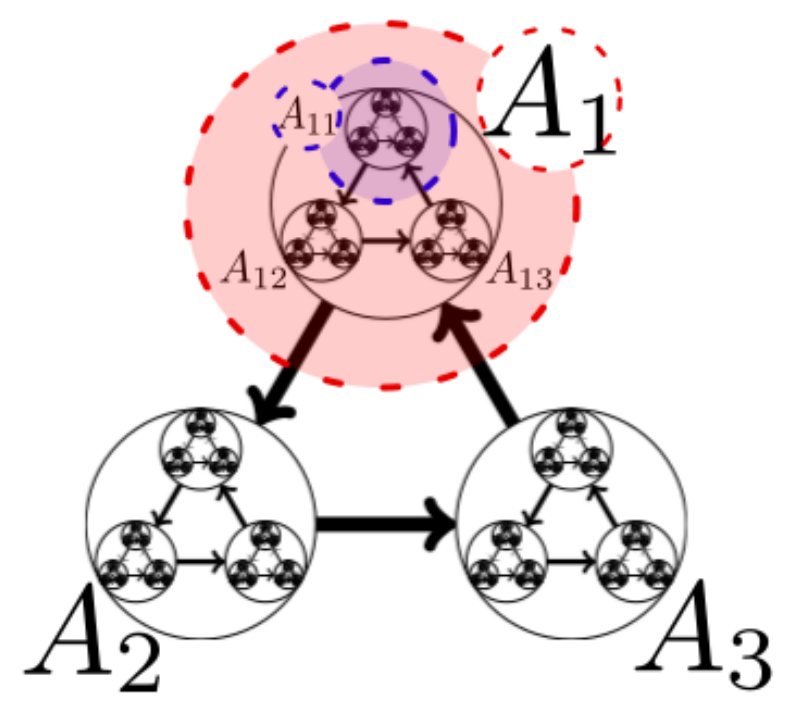

Figura 4.6: Alguns níveis de uma $\vec{C}_{3}$-decomposição do "blow-up" recursivo do $\vec{C}_{3}$.

Definição 4.6.16. Seja $T$ um torneio e $A \subset V(T)$. Definimos

$$
\begin{aligned}
& N^{+}(A)=\{v \in V(T): \forall a \in A, a v \in A(T)\} ; \\
& N^{-}(A)=\{v \in V(T): \forall a \in A, v a \in A(T)\} .
\end{aligned}
$$

Observe que $N^{+}(A) \cup N^{-}(A)$ é sempre um subconjunto de $V(T) \backslash A$ e pode ser um subconjunto próprio.

Agora vamos provar dois fatos básicos sobre torneios.

Lema 4.6.17. Se $\left(T_{n}\right)_{n \in \mathbb{N}}$ é uma sequência de torneios com $\lim _{n \rightarrow \infty}\left|T_{n}\right|=\infty$ e $c \geq 1 / 2$ é uma constante tal que pelo menos $(1-o(1))\left|T_{n}\right|$ vértices de $T_{n}$ têm grau de entrada maior que $(c+o(1))\left|T_{n}\right|$, então $c=1 / 2$.

Demonstração. Seja $\left(T_{n}^{\prime}\right)_{n \in \mathbb{N}}$ uma subsequência convergente de $\left(T_{n}\right)_{n \in \mathbb{N}}$ e $\phi \in \operatorname{Hom}^{+}\left(\mathcal{A}^{0}, \mathbb{R}\right)$ seu limite. Observe que $\boldsymbol{\phi}^{\mathbf{1}}(\beta) \geq c$ quase certamente. Pelo Teorema 4.3.12, temos $\mathbb{E}\left[\boldsymbol{\phi}^{\mathbf{1}}(\beta)\right]=1 / 2$, obtemos $c \leq 1 / 2$.

Lema 4.6.18. Seja $\left(T_{n}\right)_{n \in \mathbb{N}}$ uma sequência de torneios convergindo a um homomorfismo balanceado $\phi$, e para todo $n \in \mathbb{N}$, seja $A_{n} \subset V\left(T_{n}\right)$ tal que $\left|A_{n}\right|=\Omega\left(\left|T_{n}\right|\right)$.

Sob essas circunstâncias, se $N^{+}\left(A_{n}\right) \cup N^{-}\left(A_{n}\right)=V\left(T_{n}\right) \backslash A_{n}$ para todo $n \in \mathbb{N}$, então

$$
\left|N^{+}\left(A_{n}\right)\right|-\left|N^{-}\left(A_{n}\right)\right|=o\left(\left|T_{n}\right|\right) .
$$


Demonstração. Suponha que não. Isso significa que existe uma subsequência de $\left(T_{n}\right)_{n \in \mathbb{N}}$ e $\varepsilon>0$ tais que, possivelmente ao alterarmos todas as orientações do arcos, temos que

$$
\left|N^{+}\left(A_{n}\right)\right|-\left|N^{-}\left(A_{n}\right)\right| \geq \varepsilon\left|T_{n}\right|,
$$

para todo $n \in \mathbb{N}$.

Observe que, para todo $v \in A_{n}$, temos

$$
\begin{aligned}
\left|T_{n}\right|-2 d^{-}(v) & =d^{+}(v)-d^{-}(v)+1 \\
& =\left|N^{+}\left(A_{n}\right)\right|+d_{A_{n}}^{+}(v)-\left|N^{-}\left(A_{n}\right)\right|-d_{A_{n}}^{-}(v)+1 \\
& \geq \varepsilon\left|T_{n}\right|+d_{A_{n}}^{+}(v)-d_{A_{n}}^{-}(v)+1 \\
& =\varepsilon\left|T_{n}\right|+\left|A_{n}\right|-2 d_{A_{n}}^{-}(v) \\
& \geq(1+\varepsilon)\left|A_{n}\right|-2 d_{A_{n}}^{-}(v),
\end{aligned}
$$

onde $d_{A}^{+}(v)=\left|N^{+}(v) \cap A\right|$ e $d_{A}^{-}(v)=\left|N^{-}(v) \cap A\right|$.

Como $\phi$ é balanceado, sabemos que pelo menos $(1-o(1))\left|T_{n}\right|$ vértices de $T_{n}$ têm grau de saída $(1 / 2+o(1))\left|T_{n}\right|$, assim como $\left|A_{n}\right|=\Omega\left(\left|T_{n}\right|\right)$. Portanto se $v$ é um desses vértices, temos

$$
d_{A_{n}}^{-}(v) \geq\left(\frac{1+\varepsilon}{2}+o(1)\right)\left|A_{n}\right|
$$

Porém isto contradiz o Lema 4.6 .17 para a sequência $\left(\left.T_{n}\right|_{A_{n}}\right)_{n \in \mathbb{N}}$.

O próximo lema técnico afirma que, se uma sequência de torneios $\vec{C}_{3}$-decomponível converge a um homomorfismo balanceado, então podemos supor que pelo menos dois de $A_{1}^{(n)}, A_{2}^{(n)}$ ou $A_{3}^{(n)}$ têm tamanho não negligenciável. Adiamos a prova desse lema para a Seção 4.6.5.

Lema 4.6.19. Se $\left(T_{n}\right)_{n \in \mathbb{N}}$ é uma sequência de torneios $\vec{C}_{3}$-decomponível que converge para um homomorfismo balanceado $\phi \in \operatorname{Hom}^{+}\left(\mathcal{A}^{0}, \mathbb{R}\right)$, então existe uma sequência $\left(T_{n}^{\prime}\right)_{n \in \mathbb{N}}$ de torneios $\vec{C}_{3}$-decomponíveis e para todo $n \in \mathbb{N}$ uma $\vec{C}_{3}$-decomposição $\left(A_{\sigma}^{(n)}\right)_{\sigma \in \Sigma^{*}}$ de $T_{n}^{\prime}$ tal que

- Existe uma subsequência $\left(T_{k_{n}}\right)_{n \in \mathbb{N}}$ de $\left(T_{n}\right)_{n \in \mathbb{N}}$ tal que o torneio $T_{n}^{\prime}$ pode ser obtido de $T_{k_{n}}$ pela inversão de $o\left(\left|T_{k_{n}}\right|^{2}\right)$ arcos (por isso $\left(T_{n}^{\prime}\right)_{n \in \mathbb{N}}$ também converge para $\phi$ );

- Temos $\left|A_{1}^{(n)}\right|=\Omega\left(\left|T_{n}^{\prime}\right|\right)$ e $\left|A_{2}^{(n)}\right|=\Omega\left(\left|T_{n}^{\prime}\right|\right)$. 
Lema 4.6.20. Temos $L_{4} \Longrightarrow L_{5}$.

Demonstração. Suponha que $\phi$ é balanceado e $\vec{C}_{3}$-decomponível. Seja $T_{\vec{C}_{3}}$ a teoria universal dos torneios $\vec{C}_{3}$-decomponíveis ${ }^{5}$, i.e., só consideramos os torneios $\vec{C}_{3}$-decomponíveis e, pelo Teorema 4.6.10, só consideramos o conjunto de todos os torneios que não têm cópias de $T_{5}^{8}, T_{5}^{10}$ nem de $T_{5}^{12}$.

Observe que $\phi$ também pode ser pensado como um elemento de $\operatorname{Hom}^{+}\left(\mathcal{A}^{0}\left[T_{\vec{C}_{3}}\right], \mathbb{R}\right)$. Isso significa que existe uma sequência $\left(T_{n}^{(0)}\right)_{n \in \mathbb{N}}$ de torneios $\vec{C}_{3}$-decomponíveis que convergem para $\phi$ (o que é, por definição, uma sequência de torneios 0-igualmente $\vec{C}_{3}$-decomponíveis). Ademais podemos também supor sem perda de generalidade que $\left|T_{n}^{(0)}\right|$ é uma potência de 3 para todo $n \in \mathbb{N}$.

Agora construiremos por indução em $k$ a sequência $\left(T_{n}^{(k)}\right)_{n \in \mathbb{N}}$ de torneios $k$-igualmente $\vec{C}_{3^{-}}$ decomponíveis que convergem para $\phi$ preservando a propriedade de que $\left|T_{n}^{(k)}\right|$ é uma potência de 3 para todo $n \in \mathbb{N}$.

Suponha $k>0$ e que já temos construída $\left(T_{n}^{(k-1)}\right)_{n \in \mathbb{N}}$. Aplicando o Lema 4.6 .19 um total de $3^{k-1}$ vezes no torneio induzido pelo $(k-1)$-ésimo nível da $\vec{C}_{3}$-decomposição dos $T_{n}^{(k-1)}$, sabemos que existe uma sequência $\left(T_{n}^{\prime}\right)_{n \in \mathbb{N}}$ de torneios $\vec{C}_{3}$-decomponíveis e para todo $n \in \mathbb{N}$ existe uma $\vec{C}_{3}$-decomposição $A^{(n)}=\left(A_{\sigma}^{(n)}\right)_{\sigma \in \Sigma^{*}}$ de $T_{n}^{\prime}$ tal que

- Para todo $t \leq k-1$, temos $\Delta_{t}\left(A^{(n)}\right)=0$. Veja definição de $\Delta_{t}$ na Equação (4.8) e observe que isso vale devido a $\left|T_{n}\right|$ ser potência de 3 ;

- Existe uma subsequência $\left(T_{m_{n}}^{(k-1)}\right)_{n \in \mathbb{N}}$ de $\left(T_{n}^{(k-1)}\right)_{n \in \mathbb{N}}$ tal que o torneio $T_{n}^{\prime}$ pode ser obtido de $T_{m_{n}}^{(k-1)}$ pela inversão de $o\left(\left|T_{m_{n}}^{(k-1)}\right|^{2}\right)$ arcos, todos completamente contidos dentro de um dos conjuntos $A_{\sigma}^{(n)}$ para algum $\sigma \in \Sigma^{*} \operatorname{com}|\sigma|=k-1$;

- Para todo $\sigma \in \Sigma^{*} \operatorname{com}|\sigma|=k-1$, temos

$$
\left|A_{\sigma 1}^{(n)}\right|=\Omega\left(\left|T_{n}^{\prime}\right|\right) ; \quad\left|A_{\sigma 2}^{(n)}\right|=\Omega\left(\left|T_{n}^{\prime}\right|\right)
$$

\footnotetext{
${ }^{5}$ Quando geramos a álgebra de flag sob a teoria $T_{\vec{C}_{3}}$, estamos levando em consideração apenas os torneios que são $\vec{C}_{3}$-decomponíveis e utilizaremos a notação $\mathcal{A}^{\sigma}\left[T_{\vec{C}_{3}}\right]$, para um dado $\sigma$.
} 
Fixe $\sigma \in \Sigma^{*} \operatorname{com}|\sigma|=k-1$ e observe que pela $\vec{C}_{3}$-decomposição

$$
\begin{aligned}
& N^{+}\left(A_{\sigma 1}^{(n)}\right) \cup N^{-}\left(A_{\sigma 1}^{(n)}\right)=V\left(T_{n}^{\prime}\right) \backslash A_{\sigma 1}^{(n)} ; \\
& N^{+}\left(A_{\sigma 2}^{(n)}\right) \cup N^{-}\left(A_{\sigma 2}^{(n)}\right)=V\left(T_{n}^{\prime}\right) \backslash A_{\sigma 2}^{(n)} .
\end{aligned}
$$

Ademais, como $\Delta_{t}\left(A^{(n)}\right)=0$ para todo $t \leq k-1$, também temos

$$
\begin{aligned}
& \left|N^{+}\left(A_{\sigma 1}^{(n)}\right)\right|-\left|N^{-}\left(A_{\sigma 1}^{(n)}\right)\right|=\left|A_{\sigma 2}^{(n)}\right|-\left|A_{\sigma 3}^{(n)}\right| ; \\
& \left|N^{+}\left(A_{\sigma 2}^{(n)}\right)\right|-\left|N^{-}\left(A_{\sigma 2}^{(n)}\right)\right|=\left|A_{\sigma 3}^{(n)}\right|-\left|A_{\sigma 1}^{(n)}\right| .
\end{aligned}
$$

Aplicando o Lema 4.6 .18 para $\left(A_{\sigma 1}^{(n)}\right)_{n \in \mathbb{N}}$ e $\left(A_{\sigma 2}^{(n)}\right)_{n \in \mathbb{N}}$, obtemos

$$
\begin{gathered}
\left|A_{\sigma 2}^{(n)}\right|-\left|A_{\sigma 3}^{(n)}\right|=o\left(\left|T_{n}^{\prime}\right|\right) ; \\
\left|A_{\sigma 3}^{(n)}\right|-\left|A_{\sigma 1}^{(n)}\right|=o\left(\left|T_{n}^{\prime}\right|\right) .
\end{gathered}
$$

Como $\sigma$ foi escolhido arbitrariamente, concluímos que $\Delta_{k}\left(A^{(n)}\right)=o\left(\left|T_{n}^{\prime}\right|\right)$. Isso significa que podemos modificar $o\left(\left|T_{n}^{\prime}\right|^{2}\right)$ arcos de $T_{n}^{\prime}$ e obtemos um torneio $T_{n}^{(k)} k$-igualmente $\vec{C}_{3^{-}}$ decomponível (observe que para isto é crucial que $\left|T_{n}^{\prime}\right|$ seja uma potência de 3) e como isto não afeta convergência da sequência $\left(T_{n}^{\prime}\right)_{n \in \mathbb{N}}$, a sequência $\left(T_{n}^{(k)}\right)_{n \in \mathbb{N}}$ também converge para $\phi$ e ainda temos que $\left|T_{n}^{(k)}\right|$ é uma potência de 3 .

Lema 4.6.21. Temos $L_{5} \Longrightarrow L_{1}$.

Demonstração. Para todo $k \in \mathbb{N}$, seja $\left(T_{n}^{(k)}\right)_{n \in \mathbb{N}}$ uma sequência de torneios que são $k$-igualmente $\vec{C}_{3}$-decomponíveis que convergem para $\phi$

Nosso objetivo é diagonalizar a família das sequências $\left(T_{n}^{(k)}\right)_{n \in \mathbb{N}}$ de forma que resulte numa sequência que ainda convirja para $\phi$. Para fazer isso, façamos $\left(D_{t}\right)_{t \in \mathbb{N}}$ uma enumeração dos conjuntos com todos os torneios finitos $\mathcal{T}$, estabelecemos $f(0)=0$, e para todo $k>0$, façamos

$$
f(k)=\min \left\{u \in \mathbb{N}:\left|T_{u}^{(k)}\right|>\left|T_{f(k-1)}^{(k-1)}\right| \text { e } \forall t \leq k, \forall m \geq u,\left|p\left(D_{t} ; T_{m}^{(k)}\right)-\phi\left(D_{t}\right)\right|<\frac{1}{k}\right\} .
$$

Observe que o fato de que $\left(T_{n}^{(k)}\right)_{n \in \mathbb{N}}$ converge para $\phi$ garante que $f(k)<\infty$ para todo $k \in \mathbb{N}$.

Agora definamos a sequência de torneios $\left(U_{n}\right)_{n \in \mathbb{N}}$ fazendo $U_{n}=T_{f(n)}^{(n)}$ para todo $n \in \mathbb{N}$. 
Afirmamos que $\left(U_{n}\right)_{n \in \mathbb{N}}$ também converge para $\phi$. De fato, se $T^{\prime} \in \mathcal{T}$ é um torneio, então existe $t \in \mathbb{N}$ tal que $D_{t}=T^{\prime}$, consequentemente, para todo $n>t$, temos

$$
\left|p\left(D_{t} ; U_{n}\right)-\phi\left(D_{t}\right)\right|<\frac{1}{n}
$$

o que implica que $\lim _{n \rightarrow \infty} p\left(D_{t} ; U_{n}\right)=\phi\left(D_{t}\right)$. Desta forma $\left(U_{n}\right)_{n \in \mathbb{N}}$ converge para $\phi$.

Pela construção, sabemos que $U_{n}$ é $n$-igualmente $\vec{C}_{3}$-decomponível. Isso significa que podemos obter $\vec{C}_{\left|U_{n}\right|}^{3}$ de $U_{n}$. Como $\left|A\left(\vec{C}_{\left|U_{n}\right|}^{3}\right)\right|=\sum_{i=1}^{n} 3^{i}\left(\frac{\left|U_{n}\right|}{3^{i}}\right)^{2}$, então temos que alterar, no máximo,

$$
\left(\begin{array}{c}
\left|U_{n}\right| \\
2
\end{array}\right)-\sum_{i=1}^{n} 3^{i}\left(\frac{\left|U_{n}\right|}{3^{i}}\right)^{2}=\left(\begin{array}{c}
\left|U_{n}\right| \\
2
\end{array}\right)-\left|U_{n}\right|^{2}\left(\frac{1-3^{-n}}{2}\right)=o\left(\left|U_{n}\right|^{2}\right)
$$

$\operatorname{arcos}$ de $U_{n}$.

Portanto a sequência $\left(\vec{C}_{\left|U_{n}\right|}^{3}\right)_{n \in \mathbb{N}}$ também converge para $\phi$, e como essa é um subsequência de $\left(\vec{C}_{n}^{3}\right)_{n \in \mathbb{N}}$, temos $\phi=\phi_{\vec{C}_{3}}$.

Finalmente, vamos provar a convergência da sequência $\left(\vec{C}_{n}^{3}\right)_{n \in \mathbb{N}}$. Essa prova pode ser obtida reinterpretando as provas dos Lemas 4.6.20 e 4.6.21.

Proposição 4.6.22. A sequência $\left(\vec{C}_{n}^{3}\right)_{n \in \mathbb{N}}$ é convergente.

Demonstração. Seja

$$
\mathcal{C}=\left\{I \subset \mathbb{N}:\left(\vec{C}_{i}^{3}\right)_{i \in I} \text { is convergente }\right\}
$$

e para todo $I \in \mathcal{C}$, denotamos $\phi_{I}$ como o limite de $\left(\vec{C}_{i}^{3}\right)_{i \in I}$.

Da compacidade de $[0,1]^{\mathcal{T}}$, sabemos que $\mathcal{C} \neq \varnothing$. Ainda mais, da compacidade de $[0,1]^{\mathcal{T}}$, sabemos que existe $I_{0} \in \mathcal{C}$ tal que

$$
I_{0} \subset\left\{3^{n}: n \in \mathbb{N}\right\}
$$

Observe que se $I \in \mathcal{C}$, então $\phi_{I}$ é $\vec{C}_{3}$-decomponível (os $\vec{C}_{n}^{3}$ são $\vec{C}_{3}$-componíveis) e balanceados (todos os vértices de $\vec{C}_{3}$ têm grau de saída ou $\left\lfloor\left|\vec{C}_{n}^{3}\right| / 2\right\rfloor$ ou $\left\lceil\left|\vec{C}_{n}^{3}\right| / 2\right\rceil$ ). Portanto $\phi_{I}$ satisfaz $L_{4}$, para todo $I \in \mathcal{C}$.

Agora repetimos a prova do Lema 4.6.20 para cada $I \in \mathcal{C}$ para obter sequências $\left(T_{n}^{(k)}\right)_{n \in \mathbb{N}}$ de 
torneios $k$-igualmente $\vec{C}_{3}$-decomponíveis que convergem para $\phi_{I}$ para cada $k \in \mathbb{N}$. Entretanto, exigimos que estas sequências sejam tais que $\left|T_{n}^{(k)}\right| \in I_{0}$ para todo $n, k \in \mathbb{N}$.

Procedemos então para a prova do Lema 4.6.21 e temos que $\phi_{I}$ é também o limite da subsequência de $\left(\vec{C}_{i}^{3}\right)_{i \in I_{0}}$, consequentemente $\phi_{I}=\phi_{I_{0}}$ para todo $I \in \mathcal{C}$.

Portanto, toda subsequência convergente de $\left(\vec{C}_{n}^{3}\right)_{n \in \mathbb{N}}$ converge para o mesmo limite $\phi_{I_{0}}$. Novamente por compacidade de $[0,1]^{\mathcal{T}}$, isto implica que $\left(\vec{C}_{n}^{3}\right)_{n \in \mathbb{N}}$ é convergente.

\subsubsection{Prova do Teorema 4.6.10}

Por conveniência ao leitor, decidimos colocar o enunciado do teorema novamente abaixo.

Theorem. Um torneio $T$ é $\vec{C}_{3}$-decomponível se, e somente se, não contém cópias de $T_{5}^{8}, T_{5}^{10}$ e $T_{5}^{12}$.

Demonstração. É direto verificar que $T_{5}^{8}, T_{5}^{10}$ e $T_{5}^{12}$ não são $\vec{C}_{3}$-decomponíveis e que a propriedade de $\vec{C}_{3}$-decomponibilidade é hereditária (i.e., todo subtorneio de um torneio $\vec{C}_{3}$-decomponível é também $\vec{C}_{3}$-decomponível). Isto conclui a prova para uma direção.

Provaremos a outra direção pela indução no tamanho $n$ do torneio $T$ sem cópias de $T_{5}^{8}, T_{5}^{10}$ nem de $T_{5}^{12}$. Se $n \leq 2$, então trivialmente $T$ é $\vec{C}_{3}$-decomponível. Então, seja $n \geq 3$ e suponha que a afirmação é verdadeira para torneios com tamanho menor que $n$.

Se $T$ é transitivo, então fazemos $A$ ser o único vértice de $T$ com grau máximo de saída. Sejam $B=V(T) \backslash A$ e $C=\varnothing$, e observe que $(A, B, C)$ satisfaz os itens da Definição 4.6.8 (usando a hipótese indutiva para o item (iv)), consequentemente $T$ é $\vec{C}_{3}$-decomponível.

Suponha então que $T$ não é transitivo e sejam $a, b, c \in V(T)$ tais que $a b, b c, c a \in A(T)$. Defina os seguintes conjuntos:

$$
\begin{array}{rlrl}
V_{a b c} & =\{v \in V(T): v a, v b, v c \in A(T)\} ; & & V_{a b}=\{v \in V(T): v a, v b, c v \in A(T)\} ; \\
V_{b c} & =\{v \in V(T): a v, v b, v c \in A(T)\} ; & V_{a c}=\{v \in V(T): v a, b v, v c \in A(T)\} ; \\
V_{a}=\{v \in V(T): v a, b v, c v \in A(T)\} ; & V_{b}=\{v \in V(T): a v, v b, c v \in A(T)\} ; \\
V_{c}=\{v \in V(T): a v, b v, v c \in A(T)\} ; & V_{\varnothing}=\{v \in V(T): a v, b v, c v \in A(T)\} ;
\end{array}
$$

e observe que esses conjuntos formam uma partição de $V(T) \backslash\{a, b, c\}$. Além disso, podemos supor que $(a, b, c)$ é escolhido de uma forma tal que minimiza $\left|V_{a b c} \cup V_{\varnothing}\right|$. Afirmamos agora as 
seguintes asserções (veja Figura 4.7).

a. $V_{a b} \times V_{b c}, V_{b c} \times V_{a c}, V_{a c} \times V_{a b} \subset A(T)$, caso contrário existiria uma cópia de $T_{5}^{10}$ em $T$;

b. $V_{a} \times V_{b}, V_{b} \times V_{c}, V_{c} \times V_{a} \subset A(T)$, caso contrário existiria uma cópia de $T_{5}^{10}$ em $T$;

c. $V_{a} \times V_{a b}, V_{b} \times V_{b c}, V_{c} \times V_{a c} \subset A(T)$, caso contrário existiria uma cópia de $T_{5}^{8}$ em $T$;

d. $V_{a b} \times V_{c}, V_{b c} \times V_{a}, V_{a c} \times V_{b} \subset A(T)$, caso contrário existiria uma cópia de $T_{5}^{12}$ em $T$;

e. $V_{a b c} \times\left(V_{a} \cup V_{b} \cup V_{c}\right) \subset A(T)$, caso contrário existiria uma cópia de $T_{5}^{10}$ em $T$;

f. $V_{a b c} \times\left(V_{a b} \cup V_{b c} \cup V_{a c}\right) \subset A(T)$, caso contrário existiria uma cópia de $T_{5}^{8}$ em $T$;

g. $\left(V_{a} \cup V_{b} \cup V_{c}\right) \times V_{\varnothing} \subset A(T)$, caso contrário existiria uma cópia de $T_{5}^{8}$ em $T$;

h. $\left(V_{a b} \cup V_{b c} \cup V_{a c}\right) \times V_{\varnothing} \subset A(T)$, caso contrário existiria uma cópia de $T_{5}^{10}$ em $T$.

Agora afirmamos que $V_{a b c} \times V_{\varnothing} \subset A(T)$. Suponha que não, isto é, suponha que $v_{a b c} \in V_{a b c}$ e $v_{\varnothing} \in V_{\varnothing}$ são tais que $v_{\varnothing} v_{a b c} \in A(T)$. Como $v_{a b c} a, a v_{\varnothing} \in A(T)$, temos

$\left\{v \in V(T): v a, v v_{a b c}, v v_{\varnothing} \in A(T)\right\} \cup\left\{v \in V(T): a v, v_{a b c} v, v_{\varnothing} v \in A(T)\right\} \subset\left(V_{a b c} \cup V_{\varnothing}\right) \backslash\left\{v_{a b c}, v_{\varnothing}\right\}$,

contradizendo a escolha de $(a, b, c)$ tal que minimize $\left|V_{a b c} \cup V_{\varnothing}\right|$. Portanto, temos $V_{a b c} \times V_{\varnothing} \subset A(T)$.

A Figura 4.8 mostra todos os arcos de $T$ provados até o momento.

Finalmente, consideremos três casos.

Se $V_{a b c} \neq \varnothing$, sejam $A=V_{a b c}, B=V(T) \backslash V_{a b c}$ e $C=\varnothing$, e observe que $(A, B, C)$ satisfazem os itens na Definição 4.6.8 (usando a hipótese indutiva para o item (iv)), consequentemente $T$ é $\vec{C}_{3}$-decomponível.

Se $V_{\varnothing} \neq \varnothing$, sejam $A=V(T) \backslash V_{\varnothing}, B=V_{\varnothing}$ e $C=\varnothing$, e observe que $(A, B, C)$ satisfazem os itens na Definição 4.6 .8 (usando a hipótese indutiva para o item (iv)), consequentemente $T$ é $\vec{C}_{3}$-decomponível.

Finalmente, se $V_{a b c} \cup V_{\varnothing}=\varnothing$, Sejam

$$
A=\{a\} \cup V_{b} \cup V_{a b} ; \quad B=\{b\} \cup V_{c} \cup V_{b c} ; \quad C=\{c\} \cup V_{a} \cup V_{a c} ;
$$

e observe que $(A, B, C)$ satisfaz os itens na Definição 4.6 .8 (usando a hipótese indutiva para item (iv)), consequentemente $T$ é $\vec{C}_{3}$-decomponível. 


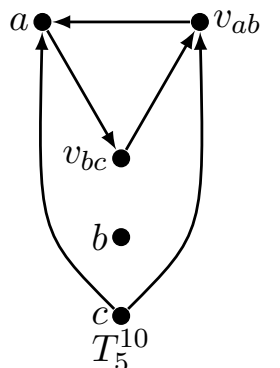

(a) Cópia de $T_{5}^{10}$ em $T$ no item (a) se $v_{a b} \in V_{a b}$ e $v_{b c} \in V_{b c}$ tal que $v_{b c}$ chega em $v_{a b}$.

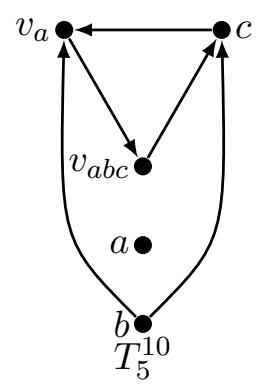

(e) Cópia de $T_{5}^{10}$ em $T$ no item (e) se $v_{a b c} \in V_{a b c}$ e $v_{a} \in V_{a}$ tal que $v_{a}$ chega em $v_{a b c}$.

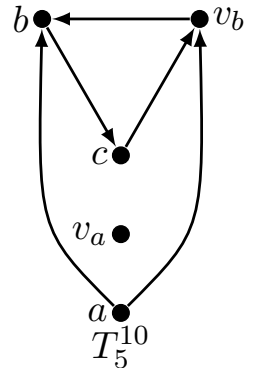

(b) Cópia de $T_{5}^{10}$ em $T$ no item (b) se $v_{a} \in V_{a}$ $e v_{b} \in V_{b}$ tal que $v_{b}$ chega em $v_{a}$.

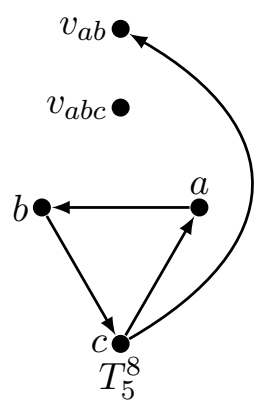

(f) Cópia de $T_{5}^{8}$ em $T$ no item (f) se $\quad v_{a b c} \in V_{a b c}$ e $v_{a b} \in V_{a b}$ tal que $v_{a b}$ chega em $v_{a b c}$.

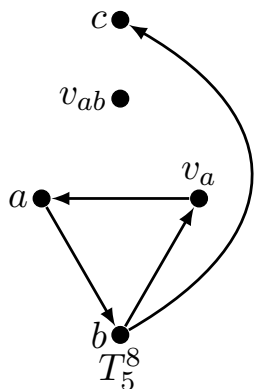

(c) Cópia de $T_{5}^{8}$ em $T$ no item (c) se $v_{a} \in V_{a}$ e $v_{a b} \in V_{a b}$ tal que $v_{a b}$ chega em $v_{a}$.

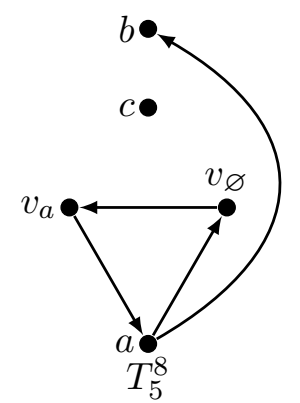

(g) Cópia de $T_{5}^{8}$ em $T$ no item (g) se $v_{a} \in V_{a}$ $e v_{\varnothing} \in V_{\varnothing}$ tal que $v_{\varnothing}$ chega em $v_{a}$.

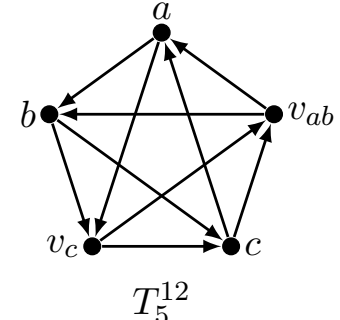

(d) Cópia de $T_{5}^{12}$ em $T$ no item (d) se $v_{a b} \in V_{a b}$ e $v_{c} \in V_{c}$ tal que $v_{c}$ chega em $v_{a b}$.

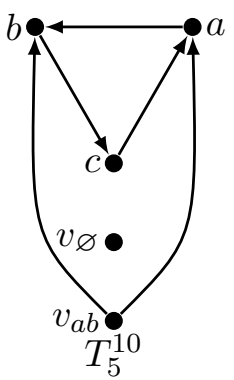

(h) Cópia de $T_{5}^{10}$ em $T$ no item (h) se $v_{a b} \in V_{a b}$ e $v_{\varnothing} \in V_{\varnothing}$ tal que $v_{\varnothing}$ chega em $v_{a b}$.

Figura 4.7: Contradições da prova do Teorema 4.6.10 envolvendo arcos entre os conjuntos $V_{a b c}, V_{a b}$, $V_{b c}, V_{a c}, V_{a}, V_{b}, V_{c}$ e $V_{\varnothing}$, e torneios proibidos $T_{5}^{8}, T_{5}^{10}$ e $T_{5}^{12}$. Os arcos omitidos são todos orientados para baixo.

\subsubsection{Prova do Lema 4.6.19}

Para conveniência do leitor, enunciaremos novamente o lema.

Lemma. Se $\left(T_{n}\right)_{n \in \mathbb{N}}$ é uma sequência de torneios $\vec{C}_{3}$-decomponíveis que converge para um homomorfismo balanceado $\phi \in \operatorname{Hom}^{+}\left(\mathcal{A}^{0}, \mathbb{R}\right)$, então existe uma sequência $\left(T_{n}^{\prime}\right)_{n \in \mathbb{N}}$ de torneios $\vec{C}_{3}$-decomponíveis e para todo $n \in \mathbb{N}$ uma $\vec{C}_{3}$-decomposição $\left(A_{\sigma}^{(n)}\right)_{\sigma \in \Sigma^{*}}$ de $T_{n}^{\prime}$ tal que

- Existe uma subsequência $\left(T_{k_{n}}\right)_{n \in \mathbb{N}}$ de $\left(T_{n}\right)_{n \in \mathbb{N}}$ tal que o torneio $T_{n}^{\prime}$ pode ser obtido de $T_{k_{n}}$ pela inversão de $o\left(\left|T_{k_{n}}\right|^{2}\right)$ arcos (por isso $\left(T_{n}^{\prime}\right)_{n \in \mathbb{N}}$ também converge para $\phi$ );

- $\operatorname{Temos}\left|A_{1}^{(n)}\right|=\Omega\left(\left|T_{n}^{\prime}\right|\right)$ e $\left|A_{2}^{(n)}\right|=\Omega\left(\left|T_{n}^{\prime}\right|\right)$.

Demonstração. Suponha que o lema não é verdade e seja $\left(T_{n}\right)_{n \in \mathbb{N}}$ um contra exemplo. 


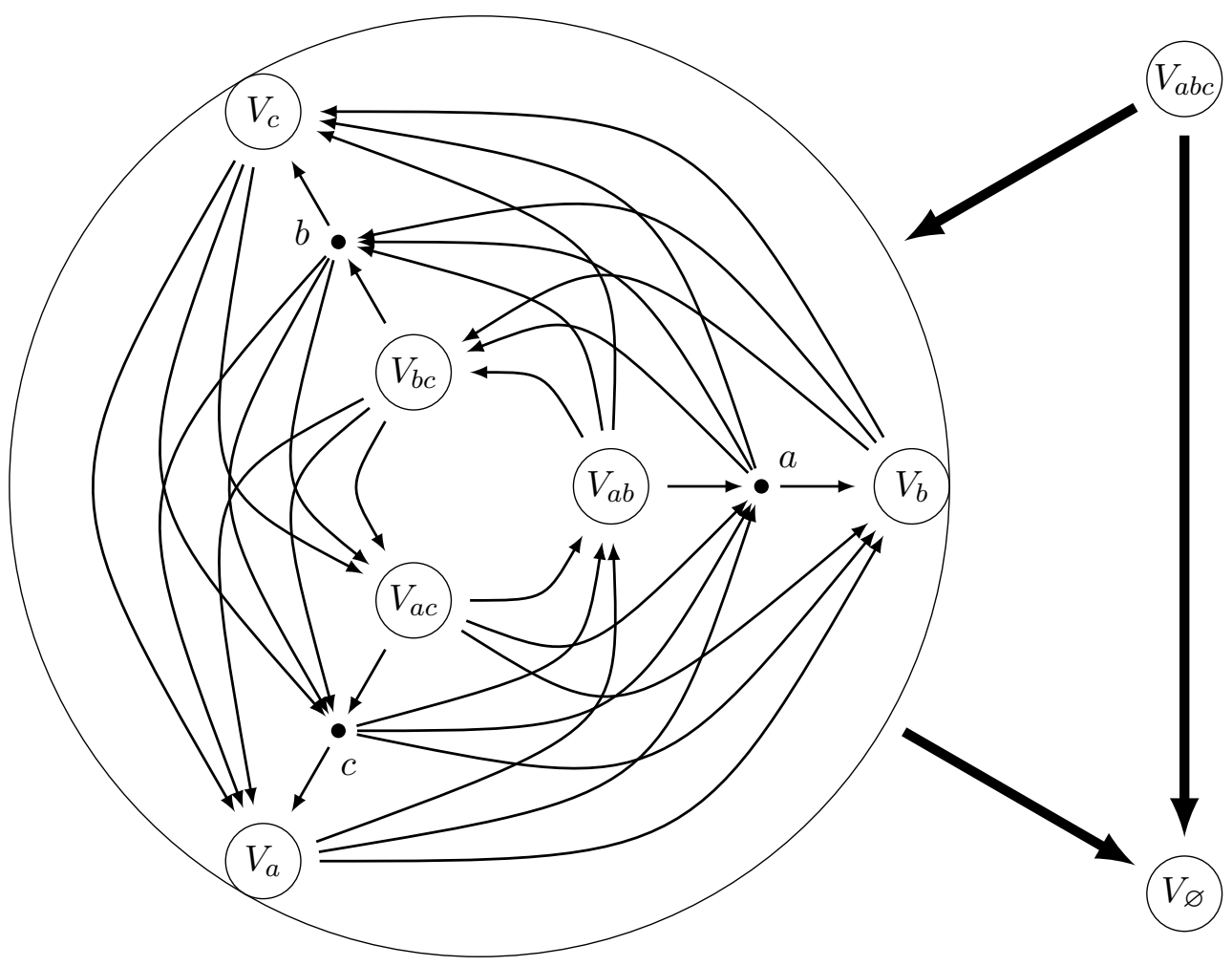

Figura 4.8: Estrutura típica de T na prova do Teorema 4.6.10.

Para todo $n \in \mathbb{N}$, seja $\left(B_{\sigma}^{(n)}\right)_{\sigma \in \Sigma^{*}}$ uma $\vec{C}_{3}$-decomposição de $T_{n}$. Sem perda de generalidade, podemos supor que

$$
\forall n \in \mathbb{N}, \forall \sigma \in \Sigma^{*},\left|B_{\sigma 1}^{(n)}\right| \geq\left|B_{\sigma 2}^{(n)}\right| \mathrm{e}\left|B_{\sigma 1}^{(n)}\right| \geq\left|B_{\sigma 3}^{(n)}\right|
$$

Afirmação 4.6.23. Suponha que $u, v: \mathbb{N} \rightarrow \mathbb{N}$ são duas funções tais que $u(n) \leq v(n)$ para todo $n \in \mathbb{N}$.

Se $\left|B_{1^{v(n)+1}}^{(n)}\right|=\Omega\left(\left|T_{n}\right|\right)$, então

$$
\left|\bigcup_{t=u(n)}^{v(n)} B_{1^{t 2}}^{(n)}\right|-\left|\bigcup_{t=u(n)}^{v(n)} B_{1^{t} 3}^{(n)}\right|=o\left(\left|T_{n}\right|\right) .
$$

Demonstração. Observe que

$$
N^{+}\left(B_{1^{v(n)+1}}^{(n)}\right)=\bigcup_{t=0}^{v(n)} B_{1^{t} 2}^{(n)} ; \quad N^{-}\left(B_{1^{v(n)+1}}^{(n)}\right)=\bigcup_{t=0}^{v(n)} B_{1^{t} 3}^{(n)} .
$$


Como $\left|B_{1^{v(n)+1}}^{(n)}\right|=\Omega\left(\left|T_{n}\right|\right)$, então pelo Lema 4.6.18, temos

$$
\left|\bigcup_{t=0}^{v(n)} B_{1^{t} 2}^{(n)}\right|-\left|\bigcup_{t=0}^{v(n)} B_{1^{t} 3}^{(n)}\right|=o\left(\left|T_{n}\right|\right) .
$$

Observe que, como $u(n) \leq v(n)$, temos $B_{1^{v(n)+1}}^{(n)} \subset B_{1^{u(n)}}^{(n)}$, o que implica $\left|B_{1^{u(n)}}^{(n)}\right|=\Omega\left(\left|T_{n}\right|\right)$, consequentemente temos

$$
\left|\bigcup_{t=0}^{u(n)-1} B_{1^{t} 2}^{(n)}\right|-\left|\bigcup_{t=0}^{u(n)-1} B_{1^{t} 3}^{(n)}\right|=o\left(\left|T_{n}\right|\right),
$$

analogamente para o caso com $v(n)$.

O resultado segue subtraindo a equação (4.10) a partir da equação (4.9).

Afirmação 4.6.24. Se $u: \mathbb{N} \rightarrow \mathbb{N}$ é uma função tal que $\left|B_{1^{u(n)+1}{ }_{2}}^{(n)}\right|=\Omega\left(\left|T_{n}\right|\right)$, então

$$
\left|\bigcup_{t=0}^{u(n)} B_{1^{t} 2}^{(n)}\right|=\Omega\left(\left|T_{n}\right|\right) .
$$

Demonstração. Suponha que a Afirmação não é verdadeira. Isso significa que existe uma subsequência $\left(T_{k_{n}}\right)_{n \in \mathbb{N}}$ de $\left(T_{n}\right)_{n \in \mathbb{N}}$ tal que

$$
\left|\bigcup_{t=0}^{u\left(k_{n}\right)} B_{1^{t} 2}^{\left(k_{n}\right)}\right|=o\left(\left|T_{k_{n}}\right|\right) .
$$

Seja $T_{n}^{\prime}$ o torneio obtido a partir de $T_{k_{n}}$ ao invertemos todos os arcos em

$$
\begin{aligned}
A\left(T_{k_{n}}\right) \cap & \left(\left(B_{1^{u\left(k_{n}\right)+13}}^{\left(k_{n}\right)} \times\left(\bigcup_{t=0}^{u\left(k_{n}\right)} B_{1^{t} 2}^{\left(k_{n}\right)}\right)\right)\right. \\
& \cup\left(\left(\bigcup_{t=0}^{u\left(k_{n}\right)} B_{1^{t} 3}^{\left(k_{n}\right)}\right) \times\left(\bigcup_{t=0}^{u\left(k_{n}\right)} B_{1^{t} 2}^{\left(k_{n}\right)}\right)\right) \\
& \left.\cup\left(\left(\bigcup_{t=0}^{u\left(k_{n}\right)} B_{1^{t} 3}^{\left(k_{n}\right)}\right) \times B_{1^{u\left(k_{n}\right)+12}}^{\left(k_{n}\right)}\right)\right)
\end{aligned}
$$

e observe que a equação (4.11) e a Afirmação 4.6.23 implicam que o total de arcos invertidos 
é $o\left(\left|T_{k_{n}}\right|^{2}\right)$. Sejam

$$
A_{1}^{(n)}=B_{1^{u\left(k_{n}\right)+2}}^{\left(k_{n}\right)} ; \quad A_{2}^{(n)}=\bigcup_{t=0}^{u\left(k_{n}\right)+1} B_{1^{t} 2}^{\left(k_{n}\right)} ; \quad A_{3}^{(n)}=\bigcup_{t=0}^{u\left(k_{n}\right)+1} B_{1^{t} 3}^{\left(k_{n}\right)}
$$

e observe que $\left|A_{1}^{(n)}\right| \geq\left|A_{2}^{(n)}\right|=\Omega\left(\left|T_{n}^{\prime}\right|\right)$.

Completando $\left(A_{1}^{(n)}, A_{2}^{(n)}, A_{3}^{(n)}\right)$ para uma $\vec{C}_{3}$-decomposição de $T_{n}^{\prime}$ contradiz a escolha de $\left(T_{n}\right)_{n \in \mathbb{N}}$ como uma sequência contra exemplo.

Afirmação 4.6.25. Se $u: \mathbb{N} \rightarrow \mathbb{N}$ é uma função tal que $\left|B_{1^{u(n)+1}}^{(n)}\right|=\Omega\left(\left|T_{n}\right|\right)$ e

$$
\left|\bigcup_{t=0}^{u(n)} B_{1^{t} 2}^{(n)}\right|=\Omega\left(\left|T_{n}\right|\right)
$$

então existe uma função $w: \mathbb{N} \rightarrow \mathbb{N}$ tal que $w(n) \leq u(n)$ e $\left|B_{1^{w(n)} 2}^{(n)}\right|=\Omega\left(\left|T_{n}\right|\right)$.

Demonstração. Para todo $n \in \mathbb{N}$, seja

$$
\begin{aligned}
& M(n)=\max \left\{\left|B_{1^{w} 2}^{(n)}\right|: w \leq u(n)\right\} ; \\
& R(n)=\max \left\{\left|\bigcup_{t=0}^{r} B_{1^{t} 2}^{(n)}\right|-\left|\bigcup_{t=0}^{r} B_{1^{t} 3}^{(n)}\right|: r \leq u(n)\right\} ; \\
& S(n)=\max \left\{\left|B_{1^{s} 3}^{(n)}\right|-\left|B_{1^{s} 2}^{(n)}\right|: s \leq u(n)\right\} .
\end{aligned}
$$

Pela Afirmação 4.6.23, sabemos que $R(n)=o\left(\left|T_{n}\right|\right)$ e $S(n)=o\left(\left|T_{n}\right|\right)$.

Suponha, no sentido de uma contradição, que a afirmação é falsa. Isso significa que devemos ter $M(n) \neq \Omega\left(\left|T_{n}\right|\right)$, isto é, existe uma subsequência $\left(T_{k_{n}}\right)_{n \in \mathbb{N}}$ de $\left(T_{n}\right)_{n \in \mathbb{N}}$ tal que $M\left(k_{n}\right)=o\left(\left|T_{k_{n}}\right|\right)$.

Observe agora que se $t \leq u\left(k_{n}\right)$ e $v_{t} \in B_{1^{t} 2}^{\left(k_{n}\right)}$ (veja Figura 4.9 mais adiante para a vizinhança do conjunto $\left.B_{1^{t} 2}^{\left(k_{n}\right)}\right)$, então

$$
\begin{aligned}
& d^{+}\left(v_{t}\right)=\left|\bigcup_{t=0}^{t-1} B_{1^{t} 2}^{\left(k_{n}\right)}\right|+\left|B_{1^{t} 3}^{\left(k_{n}\right)}\right|+d_{B_{1^{t} 2}^{\left(k_{n}\right)}}^{+}\left(v_{t}\right) ; \\
& d^{-}\left(v_{t}\right)=\left|\bigcup_{t=0}^{t-1} B_{1^{t} 3}^{\left(k_{n}\right)}\right|+\left|B_{1^{t+1}}^{\left(k_{n}\right)}\right|+d_{B_{1^{t} 2}^{\left(k_{n}\right)}}^{-}\left(v_{t}\right),
\end{aligned}
$$

onde $d_{A}^{+}(v)=\left|N^{+}(v) \cap A\right|$ e $d_{A}^{-}(v)=\left|N^{-}(v) \cap A\right|$. 
Como

$$
\begin{aligned}
\left|\bigcup_{t=0}^{t-1} B_{1^{t} 2}^{\left(k_{n}\right)}\right|-\left|\bigcup_{t=0}^{t-1} B_{1^{t} 3}^{\left(k_{n}\right)}\right| & \leq R\left(k_{n}\right) \\
\left|B_{1^{t} 3}^{\left(k_{n}\right)}\right| & \leq\left|B_{1^{t} 2}^{\left(k_{n}\right)}\right|+S\left(k_{n}\right) \leq M\left(k_{n}\right)+S\left(k_{n}\right) \\
d_{B_{1^{t} 2}^{\left(k_{n}\right)}}^{+}\left(v_{t}\right)-d_{B_{1^{t} 2}^{\left(k_{n}\right)}}^{-}\left(v_{t}\right) & \leq\left|B_{1^{t} 2}^{\left(k_{n}\right)}\right| \leq M\left(k_{n}\right) ; \\
\left|B_{1^{t+1}}^{\left(k_{n}\right)}\right| & \geq\left|B_{1^{u\left(k_{n}\right)+1}}^{\left(k_{n}\right)}\right|
\end{aligned}
$$

temos

$$
d^{+}\left(v_{t}\right)-d^{-}\left(v_{t}\right) \leq R\left(k_{n}\right)+2 M\left(k_{n}\right)+S\left(k_{n}\right)-\left|B_{1^{u\left(k_{n}\right)+1}}^{\left(k_{n}\right)}\right| \cdot
$$

Observe que esse limitante não depende de $t$.

Como $R\left(k_{n}\right)=o\left(\left|T_{k_{n}}\right|\right), M\left(k_{n}\right)=o\left(\left|T_{k_{n}}\right|\right), S\left(k_{n}\right)=o\left(\left|T_{k_{n}}\right|\right)$ e $\left|B_{1 u\left(k_{n}\right)+1}^{\left(k_{n}\right)}\right|=\Omega\left(\left|T_{k_{n}}\right|\right)$, isto implica que

$$
d^{+}(v)-d^{-}(v) \leq R\left(k_{n}\right)+2 M\left(k_{n}\right)+S\left(k_{n}\right)-\left|B_{1^{u\left(k_{n}\right)+1}}^{\left(k_{n}\right)}\right| \leq-\varepsilon\left|T_{k_{n}}\right|,
$$

Para todo $v \in \bigcup_{t=0}^{u\left(k_{n}\right)} B_{1^{t} 2}^{\left(k_{n}\right)}$ e $n \in \mathbb{N}$ suficientemente grande, o que contradiz o fato que $\phi$ é balanceado (já que $\left|\bigcup_{t=0}^{u\left(k_{n}\right)} B_{1^{t} 2}^{\left(k_{n}\right)}\right|=\Omega\left(\left|T_{k_{n}}\right|\right)$ ).

Afirmação 4.6.26. Suponha que $u, v: \mathbb{N} \rightarrow \mathbb{N}$ são duas funções tais que $u(n)<v(n)$ para todo $n \in \mathbb{N}$.

Se $\left|B_{1^{u(n)} 2}^{(n)}\right|=\Omega\left(\left|T_{n}\right|\right)$ e $\left|B_{1^{v(n)} 2}^{(n)}\right|=\Omega\left(\left|T_{n}\right|\right)$, então

$$
\left|B_{1^{u(n)} 2}^{(n)}\right|=2\left|\bigcup_{t=u(n)+1}^{v(n)-1} B_{1^{t 2}}^{(n)}\right|+3\left|B_{1^{v(n) 2} 2}^{(n)}\right|+o\left(\left|T_{n}\right|\right) .
$$

Demonstração. Pelo Lema 4.6.18, sabemos que $\left|N^{+}\left(B_{1^{v(n)} 2}^{(n)}\right)\right|-\left|N^{-}\left(B_{1^{v(n)} 2}^{(n)}\right)\right|=o\left(\left|T_{n}\right|\right)$, isto é, temos (ver Figura 4.9)

$$
\left|\bigcup_{t=0}^{v(n)-1} B_{1^{t} 2}^{(n)}\right|+\left|B_{1^{v(n)} 3}^{(n)}\right|-\left|\bigcup_{t=0}^{v(n)-1} B_{1^{t} 3}^{(n)}\right|-\left|B_{1^{v(n)+1}}^{(n)}\right|=o\left(\left|T_{n}\right|\right) .
$$




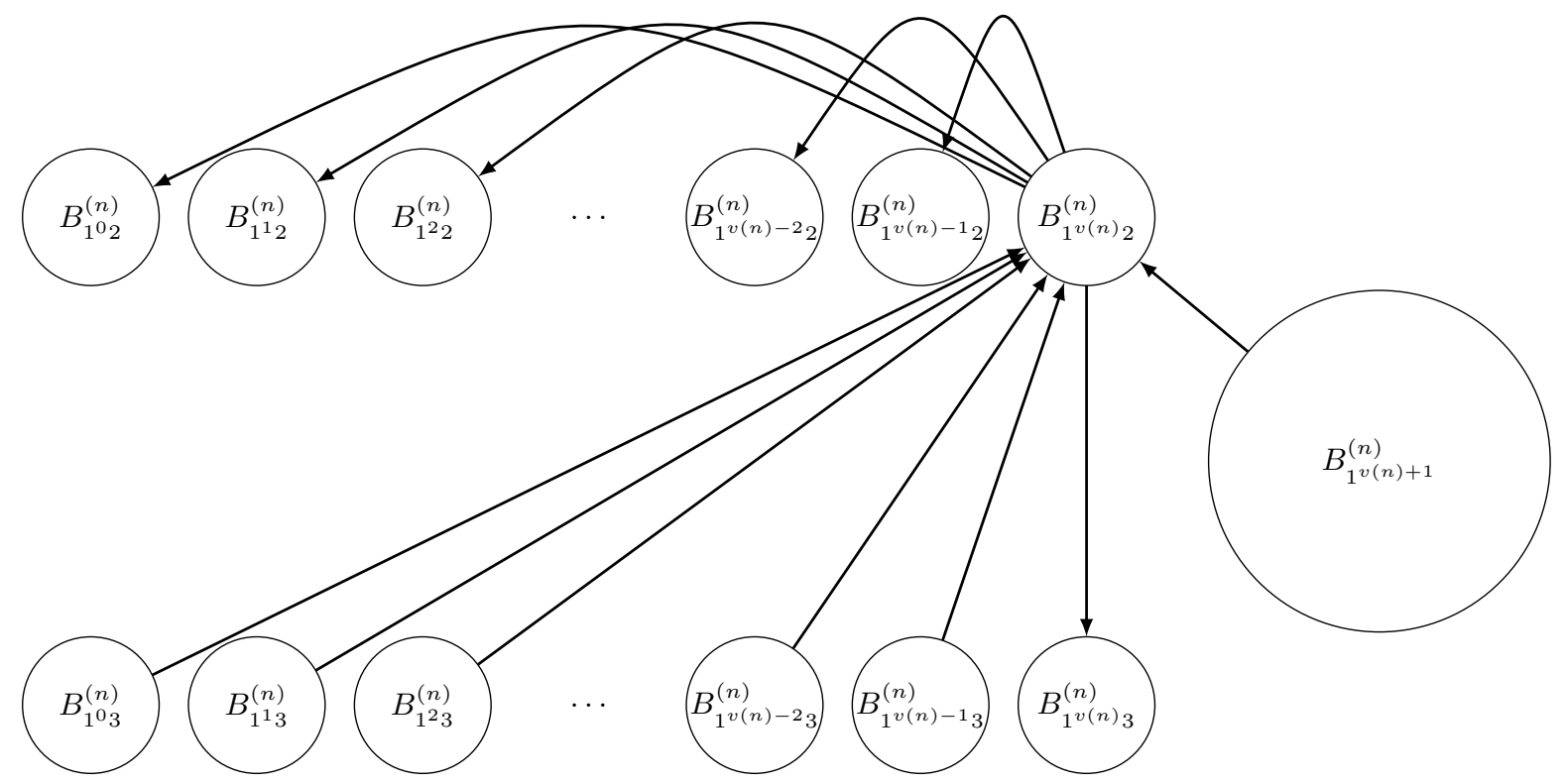

Figura 4.9: Vizinhanças de $B_{1 v(n) 2}^{(n)}$.

Pela Afirmação 4.6.23, temos

$$
\left|B_{1^{v(n)} 2}^{(n)}\right|-\left|B_{1^{v(n)+1}}^{(n)}\right|=o\left(\left|T_{n}\right|\right) .
$$

Com um argumento análogo para $u(n)$, temos

$$
\left|B_{1^{u(n)} 2}^{(n)}\right|-\left|B_{1^{u(n)+1}}^{(n)}\right|=o\left(\left|T_{n}\right|\right)
$$

o que implica

$$
\left|B_{1^{u(n)} 2}^{(n)}\right|-\left|\bigcup_{t=u(n)+1}^{v(n)-1}\left(B_{1^{t} 2}^{(n)} \cup B_{1^{t} 3}^{(n)}\right)\right|-\left|B_{1^{v(n)+1}}^{(n)}\right|-\left|B_{1^{v(n)} 2}^{(n)}\right|-\left|B_{1^{v(n)} 3}^{(n)}\right|=o\left(\left|T_{n}\right|\right) .
$$

Como $\left|B_{1^{v(n)}}^{(n)}\right| \geq\left|B_{1^{v(n)+1}}^{(n)}\right| \geq\left|B_{1^{v(n)} 2}^{(n)}\right|=\Omega\left(\left|T_{n}\right|\right)$, com mais duas aplicações da Afirmação 4.6.23 obtemos

$$
\left|B_{1^{u(n)} 2}^{(n)}\right|-2\left|\bigcup_{t=u(n)+1}^{v(n)-1} B_{1^{t} 2}^{(n)}\right|-\left|B_{1^{v(n)+1}}^{(n)}\right|-2\left|B_{1^{v(n)} 2}^{(n)}\right|=o\left(\left|T_{n}\right|\right) .
$$


Subtraindo a equação (4.12) a partir de (4.13), obtemos

$$
\left|B_{1^{u(n)} 2}^{(n)}\right|-2\left|\bigcup_{t=u(n)+1}^{v(n)} B_{1^{t} 2}^{(n)}\right|-3\left|B_{1^{v(n)} 2}^{(n)}\right|=o\left(\left|T_{n}\right|\right) .
$$

Agora temos condições de finalizar a prova do lema. Para todo $n \in \mathbb{N}$, seja

$$
v(n)=\min \left\{v \in \mathbb{N}:\left|\bigcup_{t=0}^{v}\left(B_{1^{+} 2}^{(n)} \cup B_{1^{+} 3}^{(n)}\right)\right| \geq \frac{2}{3}\left|T_{n}\right|\right\} .
$$

Observe que $v(n)$ é bem definido para $n \geq 3$ e, pela Afirmação 4.6.23, temos $\left|\bigcup_{t=0}^{v(n)} B_{1^{t} 2}^{(n)}\right|=$ $\Omega\left(\left|T_{n}\right|\right)$. Ademais, observe que

$$
\left|B_{1^{v(n)+1}}^{(n)}\right| \geq \frac{1}{9}\left|T_{n}\right|
$$

a qual, pela Afirmação 4.6.25, implica que existe uma função $w: \mathbb{N} \rightarrow \mathbb{N} \operatorname{com} w(n) \leq v(n)$ para todo $n \in \mathbb{N}$ e tal que $\left|B_{1 w(n) 2}^{(n)}\right|=\Omega\left(\left|T_{n}\right|\right)$, isto é, existem $n_{0} \in \mathbb{N}$ e $\varepsilon>0$ tais que

$$
\left|B_{1 w(n) 2}^{(n)}\right| \geq \varepsilon\left|T_{n}\right|
$$

para todo $n \geq n_{0}$.

Para todo $n \in \mathbb{N}$, seja

$$
w_{0}(n)=\min \left\{w \in \mathbb{N}:\left|B_{1^{w} 2}^{(n)}\right| \geq \varepsilon\left|T_{n}\right|\right\}
$$

e observe que, para todo $n \geq n_{0}$, temos que $w_{0}(n)$ é bem definido e $w_{0}(n) \leq w(n)$.

Como $\left|B_{1 w_{0}(n) 2}^{(n)}\right|=\Omega\left(\left|T_{n}\right|\right)$, pela Afirmação 4.6.24, sabemos que

$$
\left|\bigcup_{t=0}^{w_{0}(n)-1} B_{1^{t} 2}^{(n)}\right|=\Omega\left(\left|T_{n}\right|\right)
$$

Outra aplicação da Afirmação 4.6.25 obtemos então uma função $u: \mathbb{N} \rightarrow \mathbb{N} \operatorname{com} u(n) \leq w_{0}(n)-1$ para todo $n \in \mathbb{N}$ e tal que $\left|B_{1^{u(n)} 2}^{(n)}\right|=\Omega\left(\left|T_{n}\right|\right)$. 
Agora, pela Afirmação 4.6.26, temos

$$
\left|B_{1 u(n) 2}^{(n)}\right|=2\left|\bigcup_{t=u(n)+1}^{w_{0}(n)-1} B_{1^{t 2}}^{(n)}\right|+3\left|B_{1^{w_{0}(n) 2}}^{(n)}\right|+o\left(\left|T_{n}\right|\right) \geq 3 \varepsilon\left|T_{n}\right|+o\left(\left|T_{n}\right|\right),
$$

o que implica que para $n \in \mathbb{N}$ suficientemente grande, temos $\left|B_{1^{u(n)} 2}^{(n)}\right| \geq \varepsilon\left|T_{n}\right|$, contradizendo a definição de $w_{0}(n)$. 


\section{Capítulo 5}

\section{Considerações finais}

Neste capítulo apresentamos algumas questões que surgem dos resultados provados nos Capítulos 3 e 4 .

\subsection{Arborescência}

Da prova do Teorema 3.2.3, conjecturamos que o que determina o $\lambda(\mathcal{D}(n, p))$ é a quantidade de vértices com grau mínimo. Bal, Bennett, Cooper, Frieze e Prałat $\left[\mathrm{BBC}^{+} 16\right]$ provaram que o "hitting-time" para existência de arborescência é o mesmo para quando o digrafo aleatório contém apenas 1 vértice com grau de entrada zero.

Definimos o processo de digrafo aleatório $D_{0}, D_{1}, \ldots, D_{n(n-1)}$ como segue: para cada $m=$ $0, \ldots, n(n-1), D_{m}$ é um digrafo com conjunto de vértice $[n]$. O digrafo $D_{0}$ não contém arcos, e, para cada $1 \leq m \leq n(n-1)$, o digrafo $D_{m}$ é obtido adicionando uma nova aresta ao digrafo $D_{m-1}$ escolhida uniformemente ao acaso dentre os arcos que não estão presentes em $D_{m-1}$. Desta forma, conjecturamos a seguinte generalização.

Conjectura 5.1.1 (Hoppen-Parente-Sato). Sejam $D_{0}, \ldots, D_{n(n-1)}$ o processo de digrafo aleatório e $k$ um inteiro positivo. Temos que $\tau\left(D_{m}\right)=k$ no momento em que existem exatamente $k$ vértices $v$ tais que $d^{-}(v)=k-1=\delta^{-}$.

Também conjecturamos que a.q.c. $\tau=\lambda$ durante todo o processo do digrafo aleatório como segue.

Conjectura 5.1.2 (Hoppen-Parente-Sato). O seguinte vale no processo de digrafo aleatório $D_{0}, \ldots, D_{n(n-1)}$. a.q.c. Para todo $0 \leq m \leq n(n-1), \tau\left(D_{m}\right)=\lambda\left(D_{m}\right)$. 
Em $\left[\mathrm{BBC}^{+} 16\right]$, os autores provaram resultados de "hitting-time" envolvendo colorações de arborescências. Dizemos que um grafo (digrafo) tem coloração arco-íris se todas arestas (arcos) têm cores diferentes. Bal, Bennett, Cooper, Frieze e Prałat $\left[\mathrm{BBC}^{+} 16\right]$ provaram que o "hittingtime" para as seguintes propriedades é o mesmo para digrafos aleatórios dada uma coloração dos arcos onde cada arco recebe uma dentre $(1+o(1))$ n cores uniformemente ao acaso.

$\mathcal{Z}$ : No máximo um vértices de $\mathcal{D}(n, p)$ tem grau de entrada zero,

$\mathcal{A}: \mathcal{D}(n, p)$ tem uma arborescência,

$\mathcal{R}: \mathcal{D}(n, p)$ tem uma arborescência arco-íris.

Desse resultado, existem dois problemas interessantes. O primeiro é proposto pelos próprios autores.

Problema 5.1.3. $O$ "hitting-time" se mantém o mesmo se utilizamos apenas $n-1$ cores?

O segundo está relacionado ao Teorema 3.2.3.

Problema 5.1.4. Qual a quantidade de arborescências arco-íris disjuntas em $\mathcal{D}(n, p)$ ?

De maneira mais geral é possível estudar variações dos problemas acima, onde consideramos árvores geradores mínimas (arborescências) que possuem uma certa propriedade. Para tal, denote $T_{\mathcal{P}}(\mathcal{G}(n, p))\left(\tau_{\mathcal{P}}(\mathcal{D}(n, p))\right)$ como o valor do empacotamento de árvores geradoras (arborescências) disjuntas nas arestas (arcos) com a propriedade $\mathcal{P}$ de grafos (digrafos).

Problema 5.1.5. Dada uma propriedade $\mathcal{P}$ de grafos (digrafos), analisar o comportamento de $T_{\mathcal{P}}(\mathcal{G}(n, p))\left(\tau_{\mathcal{P}}(\mathcal{D}(n, p))\right)$.

Outro problema bastante interessante que está relacionado a empacotamentos de arborescências é estudar o comportamento de $\mathcal{D}(n, p)$ para universalidade de árvores orientadas. Dados inteiros $n$ e $\Delta^{-}$, denotamos por $\overrightarrow{\mathcal{T}}\left(n, \Delta^{-}\right)$a classe de todas as árvores orientadas com $n$ vértices e grau de entrada máximo limitado por $\Delta^{-}$. Dizemos que um digrafo $\vec{D}$ é universal para $\overrightarrow{\mathcal{T}}\left(n, \Delta^{-}\right)$se $\vec{D}$ contém todas as árvores em $\overrightarrow{\mathcal{T}}\left(n, \Delta^{-}\right)$.

Problema 5.1.6. Analisar a menor ordem de $p$ tal que $\mathcal{D}(n, p)$ é a.q.c. universal para $\overrightarrow{\mathcal{T}}\left(n, \Delta^{-}\right)$.

\subsection{Torneios}

Claramente, a primeira questão que surge dos resultados do presente trabalho é analisar a densidade assintótica máxima e sua respectiva família extremal para $T_{5}^{10}$. Utilizando o método 
semidefinido, conseguimos resultados aproximados com valores próximos a $3 / 20$, mas não conseguimos provar que a densidade máxima de $T_{5}^{10}$ é igual à $3 / 20$ nem extrair informações que pudesse nos ajudar a caracterizar a família assintótica extremal.

Conjectura 5.2.1. Para todo $\phi \in \operatorname{Hom}^{+}\left(\mathcal{A}^{0}, \mathbb{R}\right)$, temos

$$
\phi\left(T_{5}^{10}\right) \leq \frac{3}{20}
$$

Problema 5.2.2. Existe $\phi^{*} \in \operatorname{Hom}^{+}\left(\mathcal{A}^{0}, \mathbb{R}\right)$ tal que $\phi=\phi^{*}$ se, e somente se, $\phi\left(T_{5}^{10}\right)=3 / 20$.

Relembremos agora o resultado provado por Beineke e Harary [BH65] de calcular a densidade conjunta máxima dos torneios fortemente conexos

$$
\max \left\{\sum_{T \in \mathcal{S}_{k}} p\left(T ; T_{2 n+1}\right):\left|V\left(T_{2 n+1}\right)\right|=2 n+1\right\}=\sum_{T \in \mathcal{S}_{k}} p\left(T ; R_{2 n+1}\right)
$$

onde $\mathcal{S}_{k}$ denota o conjunto de todos os torneios fortemente conexos de tamanhos $k$.

É interessante observar que para a densidade conjunta, a família extremal é dada pelo homomorfismo carrossel, mas quando analisamos individualmente os torneios fortemente conexos suas famílias assintóticas extremais mudam.

Coregliano [Cor15] provou que a densidade do torneio carrossel com 4 vértices é maximizada pelo homomorfismo carrossel e propõe a seguinte conjectura que provamos para o caso de 5 vértices no Teorema 4.2.2.

Conjectura 5.2.3 (Coregliano [Cor15]). Para todo $n \geq 3$, um homomorfismo $\phi \in \operatorname{Hom}^{+}\left(\mathcal{A}^{0}, \mathbb{R}\right)$ maximiza a densidade de $R_{2 n+1}$ se e somente se $\phi=\phi_{\mathrm{R}}$.

Desta forma, é de interesse investigar a densidade individual de torneios fortemente conexos que detém uma construção bem definida e crescente na quantidade dos vértices.

Outro problema de interesse é quando desejamos encontrar a densidade assintótica de digrafos fixos em torneios. Colombo [Col64] provou que a quantidade máxima de circuitos de tamanho 4 orientados $\left(\vec{C}_{4}\right)$ é alcançado no torneio carrossel de ordem $2 n+1$. Utilizando o método semidefinido de álgebra de flags, conseguimos um resultado igual ao de Komarov e Mackey [KM14] com relação a densidade assintótica de circuito direcionado de tamanho 5 em torneios.

Seja $c(T, k)$ a quantidade de circuitos direcionados com $k$ vértices no torneio $T$, e seja 
$c(n, k)=\max \{c(T, k): T$ é um torneio com $n$ vértices $\}$. Para $k=5$, o resultado segue do Teorema 5.2.4.

Teorema 5.2.4 (Komarov-Mackey [KM14]).

$$
c(n, 5)=\frac{3}{4}\left(\begin{array}{l}
n \\
5
\end{array}\right)
$$

Ademais, Komarov e Mackey [KM14] provaram que a família dos torneios quase-aleatório é extremal para $c(n, 5)$. Pelo resultado de Coregliano e Razborov [CR16], temos que para $k=3$ o homomorfismo $\phi_{\mathrm{qr}}$ caracteriza a família extremal.

Observe que, para $k=3,5$, temos que $c(n, k)$ é maximizado pelo torneio quase-aleatório quando $k$ e, para $k=4$, pelo torneio carrossel. Então um problema natural é investigarmos a função $c(n, k)$ para $k \geq 6$ e também suas famílias extremais. Para tal podemos utilizar a teoria de álgebra de flags para extrair informações locais que nos possibilitem analisar o caso geral $c(n, k)$ para $k \geq 6$ ou outras técnicas combinatórias.

Problema 5.2.5. Para todo $k \geq 6$, analisar a função $c(n, k)$ e caracterizar sua família extremal. 
Apêndice A

\section{Matrizes positivas semidefinidas}

$$
Q\left(T_{5}^{7}, 1\right)=\frac{35}{48} \cdot\left(\begin{array}{ccccc}
\operatorname{Tr}_{3}^{1, L} & \vec{C}_{3}^{1} & \operatorname{Tr}_{3}^{1, M} & \operatorname{Tr}_{3}^{1, W} \\
1 & -1 & -1 & 1 \\
-1 & 1 & 1 & -1 \\
-1 & 1 & 1 & -1 \\
1 & -1 & -1 & 1
\end{array}\right)=\frac{35}{48} \cdot\left(\begin{array}{c}
1 \\
-1 \\
-1 \\
1
\end{array}\right) \cdot\left(\begin{array}{c}
1 \\
-1 \\
-1 \\
1
\end{array}\right) \cdot
$$




$$
\begin{aligned}
& =5 \cdot\left(\begin{array}{c}
1 \\
0 \\
-1 \\
1 \\
0 \\
1 \\
-1 \\
-1
\end{array}\right) \cdot\left(\begin{array}{c}
1 \\
0 \\
-1 \\
1 \\
0 \\
1 \\
-1 \\
-1
\end{array}\right)^{\top} \\
& Q\left(T_{5}^{7}, \vec{C}_{3}^{*}\right)=12 \cdot\left(\begin{array}{cccccccc}
R_{4}^{\vec{C}_{3}^{*}, 3} & L_{4}^{\vec{C}_{3}^{*}} & R_{4}^{\vec{C}_{3}^{*}, 2} & R_{4}^{\vec{C}_{3}^{*}, 1} & R_{4}^{\vec{C}_{3}^{*}, 23} & W_{4}^{\vec{C}_{3}^{*}} & R_{4}^{\vec{C}_{3}^{*}, 12} & R_{4}^{\vec{C}_{3}^{*}, 13} \\
0 & 0 & 0 & 0 & 0 & 0 & 0 & 0 \\
0 & 1 & 0 & 0 & 0 & -1 & 0 & 0 \\
0 & 0 & 0 & 0 & 0 & 0 & 0 & 0 \\
0 & 0 & 0 & 0 & 0 & 0 & 0 & 0 \\
0 & 0 & 0 & 0 & 0 & 0 & 0 & 0 \\
0 & -1 & 0 & 0 & 0 & 1 & 0 & 0 \\
0 & 0 & 0 & 0 & 0 & 0 & 0 & 0 \\
0 & 0 & 0 & 0 & 0 & 0 & 0 & 0
\end{array}\right) \\
& =12 \cdot\left(\begin{array}{c}
0 \\
1 \\
0 \\
0 \\
0 \\
-1 \\
0 \\
0
\end{array}\right) \cdot\left(\begin{array}{c}
0 \\
1 \\
0 \\
0 \\
0 \\
-1 \\
0 \\
0
\end{array}\right)^{\top}
\end{aligned}
$$




$$
\begin{aligned}
& Q\left(T_{5}^{8}, 1\right)=\left(\begin{array}{cccc}
\operatorname{Tr}_{3}^{1, L} & \vec{C}_{3}^{1} & \operatorname{Tr}_{3}^{1, M} & \operatorname{Tr}_{3}^{1, W} \\
\frac{2473}{6400} & -\frac{363}{1600} & -\frac{757}{1600} & \frac{2007}{6400} \\
-\frac{363}{1600} & \frac{1407}{6400} & \frac{1441}{6400} & -\frac{349}{1600} \\
-\frac{757}{1600} & \frac{1441}{6400} & \frac{4659}{6400} & -\frac{12}{25} \\
\frac{2007}{6400} & -\frac{349}{1600} & -\frac{12}{25} & \frac{2461}{6400}
\end{array}\right) \\
& Q\left(T_{5}^{8}, A\right)=\frac{99}{3200} \cdot\left(\begin{array}{cccc}
I^{A} & \vec{C}_{3}^{A} & \operatorname{Tr}_{3}^{A} & O^{A} \\
1 & -1 & -1 & 1 \\
-1 & 1 & 1 & -1 \\
-1 & 1 & 1 & -1 \\
1 & -1 & -1 & 1
\end{array}\right)=\frac{99}{3200} \cdot\left(\begin{array}{c}
1 \\
-1 \\
-1 \\
1
\end{array}\right) \cdot\left(\begin{array}{c}
1 \\
-1 \\
-1 \\
1
\end{array}\right)^{\top} \\
& Q\left(T_{5}^{8}, \operatorname{Tr}_{3}^{*}\right)=\left(\begin{array}{cccccccc}
\operatorname{Tr}_{4}^{\operatorname{Tr}_{3}^{*}, 3} & W_{4}^{\operatorname{Tr}_{3}^{*}} & \operatorname{Tr}_{4}^{\operatorname{Tr}_{3}^{*}, 2} & R_{4}^{\operatorname{Tr}_{3}^{*}, 1} & L_{4}^{\operatorname{Tr}_{3}^{*}} & \operatorname{Tr}_{4}^{\operatorname{Tr}_{3}^{*}, 1} & R_{4}^{\operatorname{Tr}_{3}^{*}, 2} & \operatorname{Tr}_{4}^{\operatorname{Tr}_{3}^{*}, 0} \\
\frac{43}{40} & \frac{43}{40} & -\frac{61}{200} & \frac{79}{100} & -\frac{241}{200} & \frac{1}{25} & -\frac{4}{5} & -\frac{37}{100} \\
-\frac{61}{200} & -\frac{131}{200} & -\frac{131}{200} & \frac{331}{100} & -\frac{1253}{200} & \frac{3}{4} & -\frac{113}{25} & -\frac{5}{4} \\
\frac{79}{100} & \frac{331}{100} & -\frac{29}{50} & \frac{187}{50} & -\frac{3}{4} & -\frac{1}{4} & \frac{7}{25} & \frac{1}{25} \\
-\frac{241}{200} & -\frac{1253}{200} & \frac{3}{4} & -\frac{113}{25} & \frac{15}{2} & -\frac{67}{100} & \frac{331}{100} & \frac{11}{10} \\
\frac{1}{25} & \frac{3}{4} & -\frac{1}{4} & \frac{7}{25} & -\frac{67}{100} & \frac{67}{100} & -\frac{27}{50} & -\frac{7}{25} \\
-\frac{4}{5} & -\frac{113}{25} & \frac{7}{25} & -\frac{223}{100} & \frac{331}{100} & -\frac{27}{50} & \frac{187}{50} & \frac{19}{25} \\
-\frac{37}{100} & -\frac{5}{4} & \frac{1}{25} & -\frac{79}{100} & \frac{11}{10} & -\frac{7}{25} & \frac{19}{25} & \frac{79}{100}
\end{array}\right) .
\end{aligned}
$$




$$
Q\left(T_{5}^{8}, \vec{C}_{3}^{*}\right)=\left(\begin{array}{cccccccc}
R_{4}^{\vec{C}_{3}^{*}, 3} & L_{4}^{\vec{C}_{3}^{*}} & R_{4}^{\vec{C}_{3}^{*}, 2} & R_{4}^{\vec{C}_{3}^{*}, 1} & R_{4}^{\vec{C}_{3}^{*}, 23} & W_{4}^{\vec{C}_{3}^{*}} & R_{4}^{\vec{C}_{3}^{*}, 12} & R_{4}^{\vec{C}_{3}^{*}, 13} \\
\frac{391}{100} & -\frac{319}{100} & \frac{13}{20} & \frac{13}{20} & -\frac{123}{50} & \frac{87}{50} & \frac{9}{50} & -\frac{37}{25} \\
-\frac{319}{100} & \frac{367}{50} & -\frac{159}{50} & -\frac{159}{50} & \frac{7}{4} & -\frac{76}{25} & \frac{7}{4} & \frac{7}{4} \\
\frac{13}{20} & -\frac{159}{50} & \frac{389}{100} & \frac{13}{20} & -\frac{37}{25} & \frac{7}{4} & -\frac{123}{50} & \frac{9}{50} \\
\frac{13}{20} & -\frac{159}{50} & \frac{13}{20} & \frac{389}{100} & \frac{9}{50} & \frac{7}{4} & -\frac{37}{25} & -\frac{123}{50} \\
-\frac{123}{50} & \frac{7}{4} & -\frac{37}{25} & \frac{9}{50} & \frac{389}{100} & -\frac{159}{50} & \frac{13}{20} & \frac{13}{20} \\
\frac{87}{50} & -\frac{76}{25} & \frac{7}{4} & \frac{7}{4} & -\frac{159}{50} & \frac{367}{50} & -\frac{159}{50} & -\frac{159}{50} \\
\frac{9}{50} & \frac{7}{4} & -\frac{123}{50} & -\frac{37}{25} & \frac{13}{20} & -\frac{159}{50} & \frac{389}{100} & \frac{13}{20} \\
-\frac{37}{25} & \frac{7}{4} & \frac{9}{50} & -\frac{123}{50} & \frac{13}{20} & -\frac{159}{50} & \frac{13}{20} & \frac{389}{100}
\end{array}\right) .
$$




$$
Q\left(T_{5}^{11}, 1\right)=\frac{5}{16} \cdot\left(\begin{array}{ccccc}
\operatorname{Tr}_{3}^{1, L} & \vec{C}_{3}^{1} & \operatorname{Tr}_{3}^{1, M} & \operatorname{Tr}_{3}^{1, W} \\
1 & -1 & -1 & 1 \\
-1 & 1 & 1 & -1 \\
-1 & 1 & 1 & -1 \\
1 & -1 & -1 & 1
\end{array}\right)=\frac{5}{16} \cdot\left(\begin{array}{c}
1 \\
-1 \\
-1 \\
1
\end{array}\right) \cdot\left(\begin{array}{c}
1 \\
-1 \\
-1 \\
1
\end{array}\right)^{\top}
$$




$$
Q\left(T_{5}^{12}, \operatorname{Tr}_{3}^{*}\right)=\frac{1}{2} \cdot\left(\begin{array}{cccccccc}
\operatorname{Tr}_{4}^{\operatorname{Tr}_{3}^{*}, 3} & W_{4}^{\operatorname{Tr}_{3}^{*}} & \operatorname{Tr}_{4}^{\operatorname{Tr}_{3}^{*}, 2} & R_{4}^{\operatorname{Tr}_{3}^{*}, 1} & L_{4}^{\operatorname{Tr}_{3}^{*}} & \operatorname{Tr}_{4}^{\operatorname{Tr}_{3}^{*}, 1} & R_{4}^{\operatorname{Tr}_{3}^{*}, 2} & \operatorname{Tr}_{4}^{\operatorname{Tr}_{3}^{*}, 0} \\
2 & 2 & -3 & 3 & -2 & 3 & -3 & -3 \\
-3 & -2 & 3 & -3 & 2 & -3 & 3 & 3 \\
3 & 2 & -3 & 3 & -2 & 3 & -3 & -3 \\
-2 & -3 & 2 & -2 & 3 & -2 & 2 & 2 \\
3 & 2 & -3 & 3 & -2 & 3 & -3 & -3 \\
-3 & -2 & 3 & -3 & 2 & -3 & 3 & 3 \\
-3 & -2 & 3 & -3 & 2 & -3 & 3 & 3
\end{array}\right) .
$$

$$
Q\left(T_{5}^{12}, \vec{C}_{3}^{*}\right)=\frac{1}{2} \cdot\left(\begin{array}{cccccccc}
R_{4}^{\vec{C}_{3}^{*}, 3} & L_{4}^{\vec{C}_{3}^{*}} & R_{4}^{\vec{C}_{3}^{*}, 2} & R_{4}^{\vec{C}_{3}^{*}, 1} & R_{4}^{\vec{C}_{3}^{*}, 23} & W_{4}^{\vec{C}_{3}^{*}} & R_{4}^{\vec{C}_{3}^{*}, 12} & R_{4}^{\vec{C}_{3}^{*}, 13} \\
7 & 5 & 7 & 7 & -7 & -5 & -7 & -7 \\
5 & 5 & 5 & 5 & -5 & -3 & -5 & -5 \\
7 & 5 & 7 & 7 & -7 & -5 & -7 & -7 \\
7 & 5 & 7 & 7 & -7 & -5 & -7 & -7 \\
-7 & -5 & -7 & -7 & 7 & 5 & 7 & 7 \\
-5 & -3 & -5 & -5 & 5 & 5 & 5 & 5 \\
-7 & -5 & -7 & -7 & 7 & 5 & 7 & 7 \\
-7 & -5 & -7 & -7 & 7 & 5 & 7 & 7
\end{array}\right)
$$




\section{Apêndice B}

\section{Polinômios característicos das}

\section{matrizes positivas semidefinidas}

$$
\begin{aligned}
P_{Q\left(T_{5}^{7}, 1\right)}(x)= & x^{4}-\frac{35}{12} x^{3} . \\
P_{Q\left(T_{5}^{7}, \mathrm{Tr}_{3}^{*}\right)}(x)= & x^{8}-30 x^{7} . \\
P_{Q\left(T_{5}^{7}, \vec{C}_{3}^{*}\right)}(x)= & x^{8}-24 x^{7} . \\
P_{Q\left(T_{5}^{8}, 1\right)}(x)= & x^{4}-\frac{55}{32} x^{3}+\frac{3450823}{10240000} x^{2}-\frac{255999851}{16384000000} x . \\
P_{Q\left(T_{5}^{8}, A\right)}(x)= & x^{4}-\frac{99}{800} x^{3} . \\
P_{Q\left(T_{5}^{8}, T_{3}^{*}\right)}(x)= & x^{8}-\frac{2549}{100} x^{7}+\frac{675593}{5000} x^{6}-\frac{149230249}{500000} x^{5}+\frac{133434036319}{400000000} x^{4} \\
& \quad-\frac{1980952353887}{10000000000} x^{3}+\frac{11839377144943}{200000000000} x^{2}-\frac{346051162035699}{50000000000000} x . \\
P_{Q\left(T_{5}^{8}, \vec{C}_{3}^{*}\right)}(x)= & x^{8}-\frac{951}{25} x^{7}+\frac{5084929}{10000} x^{6}-\frac{159696453}{50000} x^{5}+\frac{125755799203}{12500000} x^{4} \\
& \quad-\frac{1934738582639}{125000000} x^{3}+\frac{700918768199117}{62500000000} x^{2}-\frac{300346502258201}{97656250000} x . \\
P_{Q\left(T_{5}^{9}, 1\right)}(x)= & x^{4}-\frac{48}{5} x^{3}+\frac{693}{100} x^{2} . \\
P_{Q\left(T_{5}^{9}, \vec{C}_{3}^{*}\right)}(x)= & x^{8}-120 x^{7}+\frac{94608}{25} x^{6}-\frac{4478976}{125} x^{5} . \\
P_{Q\left(T_{5}^{11}, 1\right)}(x)= & x^{4}-\frac{5}{4} x^{3} . \\
P_{Q\left(T_{5}^{11}, \vec{C}_{3}^{*}\right)}(x)= & x^{8}-40 x^{7}+\frac{12828}{25} x^{6}-\frac{327744}{125} x^{5}+\frac{565056}{125} x^{4} . \\
P_{Q\left(T_{5}^{12}, 1\right)}(x)= & x^{4}-\frac{1}{4} x^{3} .
\end{aligned}
$$




$$
\begin{aligned}
& P_{Q\left(T_{5}^{12}, \operatorname{Tr}_{3}^{*}\right)}(x)=x^{8}-12 x^{7}+15 x^{6} . \\
& P_{Q\left(T_{5}^{12}, \vec{C}_{3}^{*}\right)}(x)=x^{8}-26 x^{7}+34 x^{6}-9 x^{5} .
\end{aligned}
$$




\section{Referências Bibliográficas}

[Bab11] R. Baber. Some results in extremal combinatorics. Tese de Doutorado, University College London, 2011. 58

$\left[\mathrm{BBC}^{+} 16\right]$ D. Bal, P. Bennett, C. Cooper, A. Frieze e P. Prałat. Rainbow arborescence in random digraphs. Journal of Graph Theory, 83(3):251-265, 2016. 16, 87, 88

[BES73] W. G Brown, P Erdős e M Simonovits. Extremal problems for directed graphs. Journal of Combinatorial Theory, Series B, 15(1):77 - 93, 1973. 3

[BF85] B. Bollobás e A. Frieze. On matchings and Hamiltonian cycles in random graphs. Em Random graphs '83 (Poznań, 1983), volume 118 of North-Holland Math. Stud., páginas 23-46. North-Holland, Amsterdam, 1985. 2, 15

[BH65] L. W. Beineke e F. Harary. The maximum number of strongly connected subtournaments. Canad. Math. Bull., 8:491-498, 1965. 4, 37, 38, 89

[Bol84] B. Bollobás. The evolution of random graphs. Trans. Amer. Math. Soc., 286(1):257274, 1984. 2, 15

[Bor99] B. Borchers. Csdp, a c library for semidefinite programming. Optimization Methods and Software, 11(1-4):613-623, 1999. 58

[BR13] V. Bhat e V. Rödl. Note on upper density of quasi-random hypergraphs. Electron. J. Combin., 20(2):Paper 59, 8, 2013. 40

[BS84] W. G Brown e M Simonovits. Digraph extremal problems, hypergraph extremal problems, and the densities of graph structures. Discrete Mathematics, 48(2):147 162, 1984. 3

[BSKS11] S. Ben-Shimon, M. Krivelevich e B. Sudakov. On the resilience of hamiltonicity and optimal packing of hamilton cycles in random graphs. SIAM Journal on Discrete Mathematics, 25(3):1176-1193, 2011. 2, 15

[CG90] F. Chung e R. Graham. Quasi-random hypergraphs. Random Structures Algorithms, 1(1):105-124, 1990. 40

[CG91] F. Chung e R. Graham. Quasi-random tournaments. J. Graph Theory, 15(2):173198, 1991. 40, 60, 62

[CGW88] F. Chung, R. Graham e R. Wilson. Quasirandom graphs. Proc. Nat. Acad. Sci. U.S.A., 85(4):969-970, 1988. 40

[Chu12] F. Chung. Quasi-random hypergraphs revisited. Random Structures Algorithms, 40(1):39-48, 2012. 40 
[Col64] U. Colombo. Sui circuiti nei grafi completi. Boll. Un. Mat. Ital. (3), 19:153-170, 1964. $4,37,89$

[Coo04] J. N. Cooper. Quasirandom permutations. J. Combin. Theory Ser. A, 106(1):123143, 2004. 40

[Cor01] G. Cornuéjols. Combinatorial Optimization: Packing and Covering. CBMS-NSF Regional Conference Series in Applied Mathematics. Society for Industrial and Applied Mathematics, 2001. 2, 15

[Cor15] L. N. Coregliano. Quasi-Carousel Tournaments. ArXiv e-prints, Março 2015. 40, $61,62,89$

[CPS15] L. N. Coregliano, R. F. Parente e C. M. Sato. On the maximum density of fixed strongly connected subtournaments. ArXiv e-prints, Maio 2015. 40, 41

[CR16] L. N. Coregliano e A. Razborov. On the density of transitive tournaments. Journal of Graph Theory, páginas n/a-n/a, 2016. 40, 90

[DJ08] P. Diaconis e S. Janson. Graph limits and exchangeable random graphs. Rend. Mat. Appl. (7), 28(1):33-61, 2008. 38

[ES12] G. Elek e B. Szegedy. A measure-theoretic approach to the theory of dense hypergraphs. Adv. Math., 231(3-4):1731-1772, 2012. 38

[FK08] A. Frieze e M. Krivelevich. On two Hamilton cycle problems in random graphs. Israel J. Math., 166:221-234, 2008. 2, 15

[FL16] A. Ferber e E. Long. Packing and counting arbitrary Hamilton cycles in random digraphs. ArXiv e-prints, Março 2016. 16

[FLST15] Q. Fortier, M. Léonard, Z. Szigeti e A. Talon. Old and new results on packing arborescences. Em 9th Hungarian-Japanese Symposium on Discrete Mathematics and Its Applications, Fukuoka, Japan, Maio 2015. 2

$\left[\mathrm{FNP}^{+} 15\right]$ A. Ferber, R. Nenadov, U. Peter, A. Noever e N. Škorić. Robust hamiltonicity of random directed graphs: Extended abstract. Em Proceedings of the Twenty-Sixth Annual ACM-SIAM Symposium on Discrete Algorithms, SODA '15, páginas 17521758. SIAM, 2015. 2, 16

[Fra79] A. Frank. Covering branchings. Acta Sci. Math. (Szeged), 41(1-2):77-81, 1979. 3, 18

[Fur91] Z. Furedi. Turan type problems,. Em Surveys in combinatorics 1991, volume 166 of London Math. Soc. Lecture Note Ser., páginas 253-300. Cambridge Univ. Press, Cambridge, 1991. 3

[GM98] H. N. Gabow e K. S. Manu. Packing algorithms for arborescences (and spanning trees) in capacitated graphs. Mathematical Programming, 82(1):83-109, 1998. 2

[GPGS14] P. Gao, X. Pérez-Giménez e C. M. Sato. Arboricity and spanning-tree packing in random graphs with an application to load balancing. Em Proceedings of the Twenty-Fifth Annual ACM-SIAM Symposium on Discrete Algorithms, páginas 317326. ACM, New York, 2014. 3, 12, 16, 19 
[Gri13] S. Griffiths. Quasi-random oriented graphs. J. Graph Theory, 74(2):198-209, 2013. 40

$\left[\mathrm{HKM}^{+} 13\right]$ C. Hoppen, Y. Kohayakawa, C. G. Moreira, B. Ráth e R. M. Sampaio. Limits of permutation sequences. J. Combin. Theory Ser. B, 103(1):93-113, 2013. 38

[HPS16] C. Hoppen, R. F. Parente e C. M. Sato. Packing arborescences in random digraphs. ArXiv e-prints, Maio 2016. 17

[JŁR00] S. Janson, T. Łuczak e A. Ruciński. Random graphs. Wiley-Interscience, New York, 2000. $7,11,12$

[Kam14] N. Kamiyama. Arborescence problems in directed graphs: Theorems and algorithms. Interdisciplinary Information Sciences, 20(1):51-70, 2014. 2

[Kee11] P. Keevash. Hypergraph Turán problems. Em Surveys in combinatorics 2011, volume 392 of London Math. Soc. Lecture Note Ser., páginas 83-139. Cambridge Univ. Press, Cambridge, 2011. 3

[KK15] N. Kamiyama e Y. Kawase. On packing arborescences in temporal networks. Information Processing Letters, 115(2):321 - 325, 2015. 2

[KKO15] F. Knox, D. Kühn e D. Osthus. Edge-disjoint Hamilton cycles in random graphs. Random Structures Algorithms, 46(3):397-445, 2015. 2, 15

[KM14] N. Komarov e J. Mackey. On the number of 5-cycles in a tournament. ArXiv e-prints, Outubro 2014. 89, 90

[KO14] D. Kühn e D. Osthus. Hamilton decompositions of regular expanders: applications. J. Combin. Theory Ser. B, 104:1-27, 2014. 2, 15

[KP13] D. Král' e O. Pikhurko. Quasirandom permutations are characterized by 4-point densities. Geom. Funct. Anal., 23(2):570-579, 2013. 40

[KS06] M. Krivelevich e B. Sudakov. Pseudo-random graphs. Em More Sets, Graphs and Numbers, Bolyai Society Mathematical Studies 15, páginas 199-262. SpringerVerlag, 2006. 40

[KS12] M. Krivelevich e W. Samotij. Optimal packings of Hamilton cycles in sparse random graphs. SIAM J. Discrete Math., 26(3):964-982, 2012. 2, 15

[KS13] S. Kalyanasundaram e A. Shapira. A note on even cycles and quasirandom tournaments. J. Graph Theory, 73(3):260-266, 2013. 40

[LLR15] O. Lee. e M. Leston-Rey. A faster algorithm for packing branchings in digraphs. Discrete Applied Mathematics, 194:121 - 131, 2015. 2

[LRW15] M. Leston-Rey e Y. Wakabayashi. Packing in generalized kernel systems: a framework that generalizes packing of branchings. Mathematical Programming, 149(1):209-251, 2015. 2

[LS06] L. Lovász e B. Szegedy. Limits of dense graph sequences. J. Combin. Theory Ser. $B, 96(6): 933-957,2006.38,44$ 
[Mad78] W. Mader. A reduction method for edge-connectivity in graphs. Ann. Discrete Math., 3:145-164, 1978. Advances in graph theory (Cambridge Combinatorial Conf., Trinity College, Cambridge, 1977). 2, 15

[Max14] Maxima. Maxima, a computer algebra system. version 5.34.1, 2014. 58

[Men27] K. Menger. Zur allgemeinen kurventheorie. Fundamenta Mathematicae, 10(1):96115, 1927. 2,15

[MV16] D. Mubayi e J. Verstraëte. A survey of Turán problems for expansions, páginas 117-143. Springer International Publishing, Cham, 2016. 3

[NW61] C. Nash-Williams. Edge-disjoint spanning trees of finite graphs. J. London Math. Soc., 36:445-450, 1961. 1, 15, 16

[Raz07] A. Razborov. Flag algebras. J. Symbolic Logic, 72(4):1239-1282, 2007. 4, 38, 42, $43,44,46,50$

[Raz10] A. Razborov. On 3-hypergraphs with forbidden 4-vertex configurations. SIAM J. Discrete Math., 24(3):946-963, 2010. 42, 47

[Raz13a] A. Razborov. Flag algebras: an interim report, 2013. 42

[Raz13b] A. Razborov. What is... a flag algebra? 60(10):1324-1327, 2013. 42

[Tho87] A. Thomason. Pseudo-random graphs. Ann. of Discrete Math., 33:307-331, 1987. 40

[Tut61] W. Tutte. On the problem of decomposing a graph into $n$ connected factors. $J$. London Math. Soc., 36:221-230, 1961. 1, 15, 16

$\left[\mathrm{YFF}^{+} 12\right]$ M. Yamashita, K. Fujisawa, M. Fukuda, K. Kobayashi, K. Nakata e M. Nakata. Latest developments in the SDPA family for solving large-scale SDPs. Em Handbook on semidefinite, conic and polynomial optimization, volume 166 of Internat. Ser. Oper. Res. Management Sci., páginas 687-713. Springer, New York, 2012. 58 